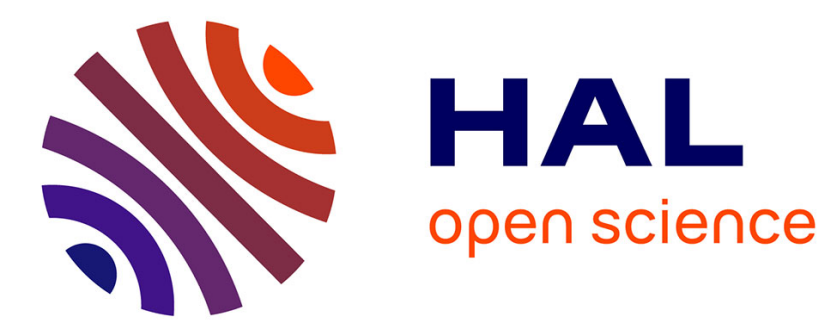

\title{
Metal-catalyzed rearrangement of allenylsulfides to furan: A theoretical mechanistic approach
}

\author{
Michel Rajzmann, Jianbo Wang, Stéphane Humbel
}

\section{To cite this version:}

Michel Rajzmann, Jianbo Wang, Stéphane Humbel. Metal-catalyzed rearrangement of allenylsulfides to furan: A theoretical mechanistic approach. Journal of Molecular Catalysis, 2017, 443, pp.148 - 154. 10.1016/j.mcat.2017.09.031 . hal-01629621

\section{HAL Id: hal-01629621 \\ https://hal.science/hal-01629621}

Submitted on 11 May 2018

HAL is a multi-disciplinary open access archive for the deposit and dissemination of scientific research documents, whether they are published or not. The documents may come from teaching and research institutions in France or abroad, or from public or private research centers.
L'archive ouverte pluridisciplinaire HAL, est destinée au dépôt et à la diffusion de documents scientifiques de niveau recherche, publiés ou non, émanant des établissements d'enseignement et de recherche français ou étrangers, des laboratoires publics ou privés. 


\title{
Metal-Catalysed Rearrangement of Allenylsulfides to Furan: A Theoretical Mechanistic Approach
}

\author{
Michel Rajzmann, ${ }^{a}$ Jianbo Wang ${ }^{b}$, Stephane Humbel, ${ }^{a}$ \\ ${ }^{a}$ Aix Marseille Univ, CNRS, Centrale Marseille, iSm2, Marseille, France \\ ${ }^{b}$ Key Laboratory of Bioorganic Chemistry and Molecular Engineering of Ministry of Education, College of Chemistry, Peking University, Beijing I0087I, \\ China
}

\author{
Keywords: \\ Molecular mechanism \\ Homogeneous catalysis \\ Transition metals \\ Cyclization \\ Allenes \\ Density functional calculations
}

\begin{abstract}
A B S T R A C T
A furan catalytic synthesis from allene is studied with quantum chemistry computations. Both specific and non-specific solvent effect are considered. The complete catalytic cycle is studied for three metals, and compared to experimental data. The mechanism is disclosed and the catalyst regeneration shows clear difference between the three metals. For Pt, the regeneration corresponds to a two-steps mechanism with a stable intermediate. For $\mathrm{Au}$ and Ru catalysts the regeneration is a one step mechanism with a low barrier only for $\mathrm{Au}(3.8 \mathrm{kcal} / \mathrm{mol})$. For $\mathrm{Au}$, the results of the computations contrasts with the experiments, hence a poisoning of the catalyst is most likely. On the contrary, in the Ru case, the regeneration corresponds to the highest transition state of the cycle.
\end{abstract}

(C) 2017 xxxxxxxx. Hosting by Elsevier B.V. All rights reserved.

\section{Introduction}

Allenes have attracted significant attentions as a consequence of their unique structural feature and chemical reactivities.[1-11]The allene moieties can coordinate with transition-metals, thus being activated. The activated allene moieties are prone to be attacked by nucleophiles, and subsequently various transformations that are highly useful in organic synthesis can be triggered. In particular, the transition-metal-catalyzed reaction of allenes bearing $\alpha$-substituents as internal nucleophililes makes it possible to develop efficient synthetic method for heterocycles. One of such transformations is the transition-metal-catalyzed cyclization of allenyl ketones that affords furans in a straightforward manner (Fig. 1). [12-17] Several metals are found in the literature as useful catalysts for this purpose. The catalyst can be for instance a calcium complex, [18-20] but electron-rich metals such as gold, platinum, and ruthenium are also used. The reaction is a cycloisomerisation with atoms or even groups transpositions.[21-23]

Furans are important heterocycles, which are present as key structure units in many biologically important natural products and pharmaceutical substances.[24] Furans are also widely used as building blacks in organic synthesis.[25] Thus, the development of novel and efficient approach to furan derivatives has been an active research area over the years.[26-34] One of the authors previously studied the transition metal-catalyzed reaction of allenic sulfides. The reaction proceeds through an intriguing 1,4-migration of thio group to afford furan derivatives, as shown in Fig. (1).[35] The reaction presumably involves a metal carbene intermediate, which is trapped intramolecularly by carbonyl group. To gain detailed

\footnotetext{
* Corresponding author at: Aix Marseille Univ, CNRS, Centrale Marseille, iSm2, Marseille, France

E-mail addresses: michel.raizmann @univ-amu.fr (M. Raizmann). stevhane.humbel@univ-amu.fr (S. Humbel)
} 
insights into the reaction mechanism of this novel transformation, we have launched a computational study on this reaction.

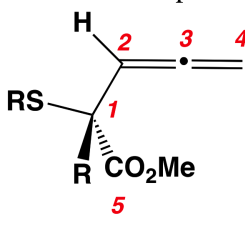

1

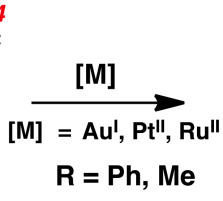

$\mathrm{R}=\mathrm{Ph}, \mathrm{Me}$

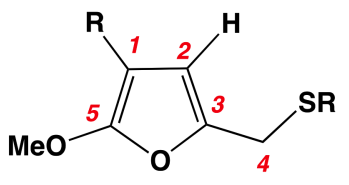

2

Fig. 1. Cycloisomerisation-transposition at work. Experimentally, Phenyls are used as R substituents. Methyls are used in computations.

Table 1. Main experimental results from ref [35].

\begin{tabular}{lccc}
\hline Catalyst $^{[\mathbf{a}]}$ & $\mathbf{T}\left[{ }^{\circ} \mathbf{C}\right]$ & $\mathbf{t}[\mathbf{h}]$ & Yield $\left[\%^{[\mathbf{b}]}\right.$ \\
\hline $\mathrm{AuCl}$ & 80 & 17 & Trace $^{[\mathrm{c}]}$ \\
$\mathrm{PtCl}_{2}$ & 80 & 4.5 & 73 \\
{$\left[\left\{\mathrm{RuCl}_{2}(\mathrm{p}\right.\right.$-cymene $\left.\left.)\right\}\right]$} & 80 & 1 & 93 \\
{$\left[\left\{\mathrm{RuCl}_{2}(\mathrm{p}\right.\right.$-cymene $\left.\left.)\right\}\right]$} & 25 & 23 & 88 \\
\hline
\end{tabular}

[a] Reactions were carried out with $5 \mathrm{~mol} \%$ catalyst. [b] Yield of isolated product after column chromatography [c] Most of the starting material was recovered.

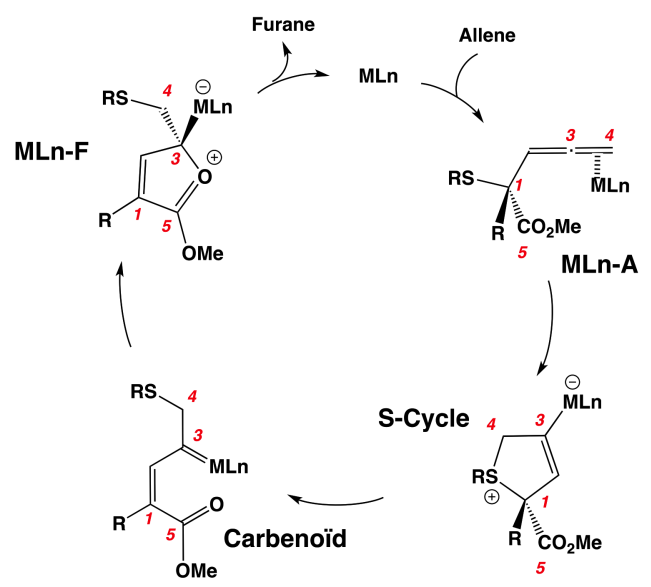

Fig. 2. Catalytic cycle proposed for the cycloisomerisation-transposition; adapted from ref [35]. Experimentally, phenyls are used as R substituents. In computations methyls are used instead.

Indeed, in the present contribution, we address the mechanism of this catalysis by computing the different intermediates and transition states summarized in Fig 2. Three MLn catalysts are studied $\mathrm{AuCl}, \mathrm{PtCl}_{2}$ and $\left\{\mathrm{RuCl}_{2}\right.$ (p-cymene $\left.\left.)\right\}\right]$. They correspond to the catalysts experimentally studied for this reaction, as reminded in Table 1. We shall thus refer explicitly to experimental results in the discussion.

Moreover, for each catalyst, a special attention will be given to the step that concerns the catalyst regeneration. Notably, the aforementioned $\mathrm{C}^{1} \rightarrow \mathrm{C}^{4}$ migration involves two ring closures and one ring opening. The postulated cyclic intermediate is named here S-Cycle: we use for the name the label of heteroatom that closes the ring. A Carbenoïd

intermediate is then postulated, and upon the $\mathrm{C}^{3}$-O bond formation, it connects to the complex formed by the furan (reaction product) and the catalyst. We refer to this complex with the label MLn-F. The so-called MLn catalyst is then restored upon Furan - Allene exchange.

\section{Methods}

The computations were done at the level M06-D3/lanl2dz(d), and we accounted for non specific solvent effects of the toluene with the standard PCM method. Specific effects were handled with one benzene molecule explicitly introduced on the metal: MLn refers to the complex with the benzene ligand. The reaction described in Fig 1 is exothermic by -24.4 $\mathrm{kcal} / \mathrm{mol}$ (when $\mathrm{R}=\mathrm{Phenyl}$ ). In order to alleviate the computations, we modeled the $\mathrm{R}=$ Phenyl substituent by $\mathrm{R}=$ Methyl throughout. With $\mathrm{R}=$ Methyl, the reaction exothermicity is smaller $(-16.5 \mathrm{kcal} / \mathrm{mol})$. Such a difference is due to the conjugation of the $\mathrm{R}=\mathrm{Phenyl}$ with the double bond $\mathrm{C}^{1}=\mathrm{C}^{5}$ formed in the product (Fig 1). Indeed, the $\mathrm{R}=$ Methyl does not conjugate, so the exothermicity is smaller in the model. Hence, the choice of this model will alter the energetics of the reaction mechanism whenever the R substituent conjugates with double bonds: the Carbenoïd, and the MLn-F intermediates would be about $7 \mathrm{kcal}^{\mathrm{mol}}{ }^{-1}$ lower when R=Phenyl compared to the model computations. We shall be watchful on that. A second simplification concerns the $\mathrm{Ru}$ catalyst: the p-cymene was modeled with a phenyl. This was done to limit the number of possible orientations of this ligand and as a priori no effect on the energetics. Our computations were done with the Gaussian g09 D01 suite of programs,[36] and the non specific solvent effect was modeled with the IEF-PCM solvation model,[37] as implemented in Gaussian. The M06/lanl2dz(d) level of calculation was used throughout, and this M06 level[38-40] was supplemented with Grimme's long range dispersion correction D3.[41-44] The "(d)" in the lan12dz(d) acronym corresponds to polarization function that we added to the standard relativistic ECP and basis set lanl2dz[45]. The $3 \mathrm{~d}$ polarization functions concerned the $3 \mathrm{~d}$ orbitals of Carbon and Oxygen with exponents $\zeta_{\mathrm{dC}}=0.75$ and $\zeta_{\mathrm{dO}}=0.85$. These values come from the exponent of the D95(d) (or DZP) basis set, as implement in Gaussian. Our computations used the chain method (CHAIN)[46,47] and AMPAC[48] to find the best paths that link MLn-A reactive to the product MLn-F. More details on the structures can be found in the experimental section. Additionally, several other paths and all the structures are available in the supplementary material (29 pages).

\subsection{Metal-Allene complexes: MLn-A Tables}

In the first step of the reaction, the metal has to bind to the allene. Upon the formation of the complex, the allene looses its quasi linearity, and bends at the central $\mathrm{C}^{3}$ atom with an angle of about $160^{\circ}$.[49-54] The stabilization is of 10.1, 34.6 and $12.2 \mathrm{kcal}^{\mathrm{mol}}{ }^{-1}$ for the $\mathrm{Au}, \mathrm{Pt}$ and $\mathrm{Ru}$ catalysts, respectively. Two orientations can be found, with the metal either exo- to the thiolate (a), or endo- (b). These two situations are reminded in Fig 3. It is noteworthy that the (a) situation allows a direct 1,4 migration of the thiolate group. The endo- orientation was also studied, and we showed that this path does not correspond to effective intermediates. A detailed study is included in the supplementary material. 


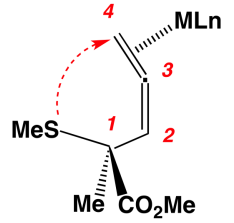

(a)

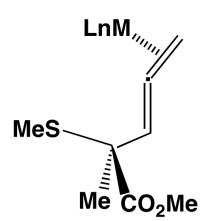

(b)
Fig. 3. The interaction with the metal leads to two possible complexes.

\subsection{Catalyst regeneration : Furane-Metal-Allene complexes}

Once the furane is formed, the catalyst has formed, F-MLn contains a metal-Carbon bond that needs to break in order to renew the catalyst and allow a new allene molecule to come in the cycle. This can be achieved through either a dissociative (Equation 1) or an associative path (Equation 2). However, the dissociation of the furane from the catalyst costs at least $14.6 \mathrm{kcal} / \mathrm{mol}$ (for $\mathrm{Au}, \mathrm{Ru}$ ), and up to $38.9 \mathrm{kcal} / \mathrm{mol}$ (Pt case). On the other hand, the approach of the allene involves at first stabilizing interactions. Finally, the associative path is always lower in energy than the dissociative.

$$
\begin{aligned}
& F-M L n+A \rightarrow F+M L n+A \rightarrow F+M L n-A \\
& F-M L n+A \rightarrow F \ldots M L n \ldots A \rightarrow F+M L n-A
\end{aligned}
$$

As we shall see, this Furane - Allene exchange that completes the catalytic cycle is of primary importance, and each catalyst behave differently also for this matter. For consistency in the energetics, the second allene molecule is added to our computation since the very first step but does not participate to the reaction until the catalyst regeneration (dispersion terms are thus included). Upon geometry optimization we verified that this additional moieties does not bind to the metal prior to the exchange (see the geometries in the supplementary information).

For the three catalysts of interest, the reaction has been studied and the results are given and discussed in the next section.

\section{Results and Discussion}

\section{1. $\mathrm{AuCl} \bullet\left\{\mathrm{C}_{6} \mathrm{H}_{6}\right\}$}

The energy profile for this Gold catalyst is shown on Fig 4. The reaction starts with an easy S-cycle formation, and the most energetic step is the actual O-C bond formation that links S-cycle to AuLn-F via TS2 (barrier height is $\left.16.0 \mathrm{kcal}^{\mathrm{mol}}{ }^{-1}\right)$. This step is also the most exothermic $(-19.7$ $\mathrm{kcal} \cdot \mathrm{mol}^{-1}$ ).

The catalyst's regeneration is a one-step process, where the exchange between the furane (to be released) and the incoming allene (for the next catalytic cycle) is made around the metal in a unique transition state (TSx). The furane is easily released when the next allene (noted $A$ in the Figures) strongly binds to the metal (TSx, corresponds to a small barrier of only $+3.8 \mathrm{kcal} . \mathrm{mol}^{-1}$ ).

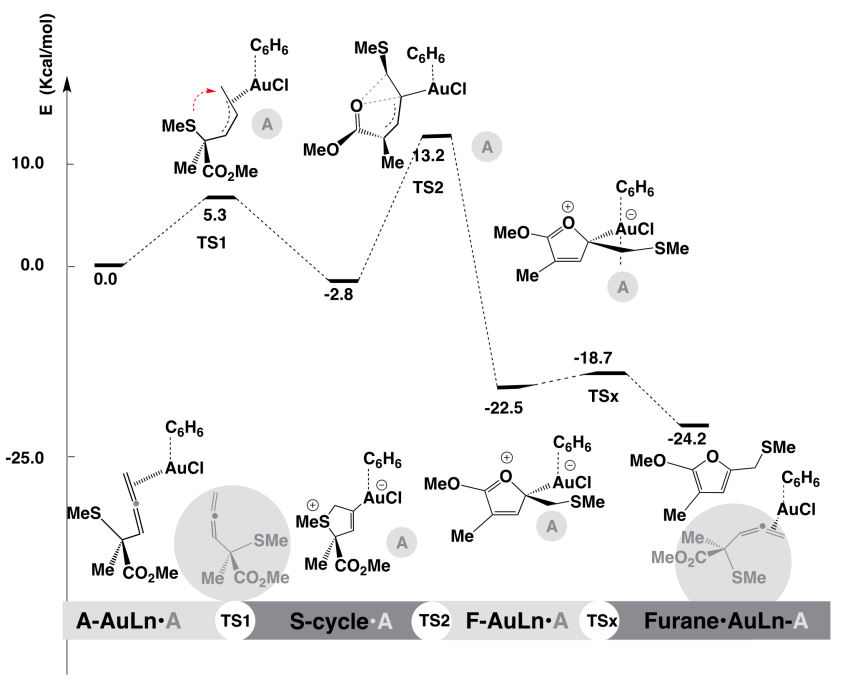

Fig. 4. Energy profile (in kcal.mol ${ }^{-1}$ ) for the $\mathrm{AuCl} \cdot\left\{\mathrm{C}_{6} \mathrm{H}_{6}\right\}$ catalyst, at the M06-D3/lan12dz(d) level. A represents the additional allene molecule.

The results we obtained show a facile reaction that should occur, and this is consistent with several cases where gold catalysts are efficiently used with similar reagents.[49,55-60] However it is in contrast with the experimental results: at $80^{\circ} \mathrm{C}$ during $17 \mathrm{~h}$, only traces of furane are obtained, and starting materials are recovered. At this temperature the highest barrier $(16 \mathrm{kcal} / \mathrm{mol})$ should be overpassed. The best hypothesis is that allene somehow reacts also stoichiometrically with the catalyst complex and modifies it (poisoning). Sulfur has indeed a strong affinity for gold clusters. Hence, we envisage that a poisoning of the catalyst could produce gold clusters that are stabilized by thiolates.[61] This hypothesis is consistent with the fact that traces of the furane product are found: the reaction is possible but the catalyst is consumed by poisoning. In the end most of the starting materials are indeed recovered.

\section{2. $\mathrm{PtCl}_{2} \bullet\left\{\mathrm{C}_{6} \mathrm{H}_{6}\right\}$}

The energy profile for the Platinium catalyst is shown on Fig 5. It differs from that of the Gold catalyst only in the catalyst's regeneration steps. The reaction also starts with an easy $\mathbf{S}$-cycle formation, and the most energetic step is the actual O-C bond formation that links S-cycle to F-PtLn via TS2 (barrier height is $14.2 \mathrm{kcal}_{\mathrm{mol}}{ }^{-1}$ ). This step is also the most exothermic $\left(-19.9 \mathrm{kcal}^{\mathrm{mol}}{ }^{-1}\right)$. For the catalyst regeneration, two steps (hence two Transition States - TSx' and $\mathbf{T S} \mathbf{x}^{\prime \prime}$ ) are necessary. They release the furane and prepare the catalyst for the next cycle.

In order to complete the catalytic cycle, the system goes through the very stable complex F-PtLn-A $(-36.6 \mathrm{kcal} / \mathrm{mol})$ where both the furane and the next allene are firmly attached to the $\mathrm{Pt}$, and the solvent, represented by the benzene, moves away, at more than $4.5 \AA$ from the metal. The release of the furane from this intermediate involves a barrier of $17.2 \mathrm{kcalmol}^{-1}$. This is the bottle-neck for this catalyst. 


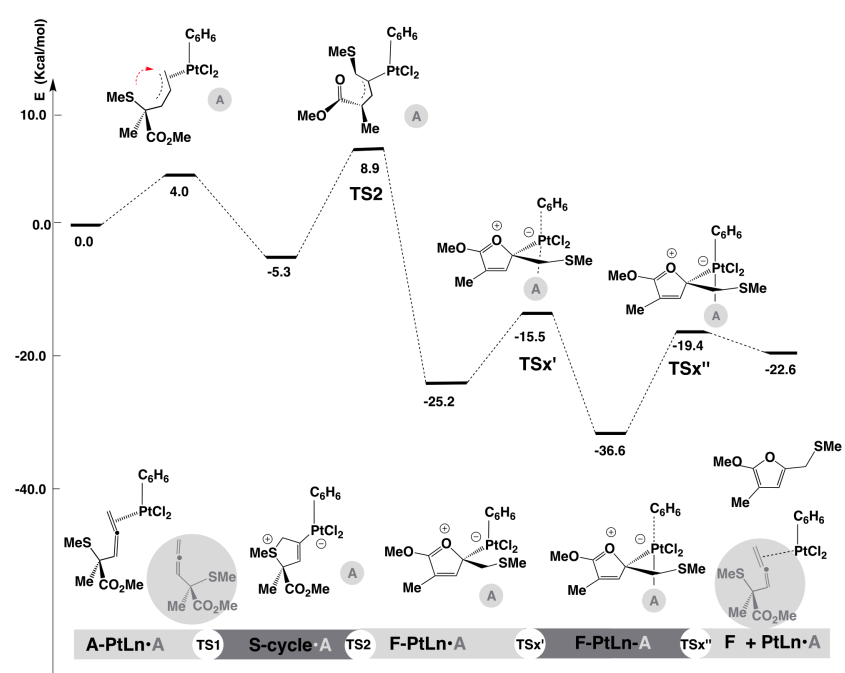

Fig. 5. Energy profile (in kcal.mol ${ }^{-1}$ ) for the $\mathrm{PtCl}_{2} \bullet\left\{\mathrm{C}_{6} \mathrm{H}_{6}\right\}$ catalyst, at the M06-D3/lanl2dz(d) level. $\mathrm{A}$ represents the additional allene molecule.

\section{3. $\mathrm{RuCl}_{2} \bullet\left\{\mathrm{C}_{6} \mathrm{H}_{6}\right\}$}

The energy profile for this catalyst is shown on Fig 6 . It is very similar to that of the Gold catalyst, with an easy furane formation (exothermic and with small barriers - about $10 \mathrm{kcal} / \mathrm{mol}$ ). Additionally, as it is the case for Gold, the catalyst's regeneration is also a one-step process, where the exchange between the furane (to be released) and the incoming allene (for the next catalytic cycle) is made around the metal in a unique transition state (TSx). The release of the furane from the latest intermediate involves a barrier of about a $13.5 \mathrm{kcalmol}^{-1}$. This is the highest barrier of the catalytic cycle.

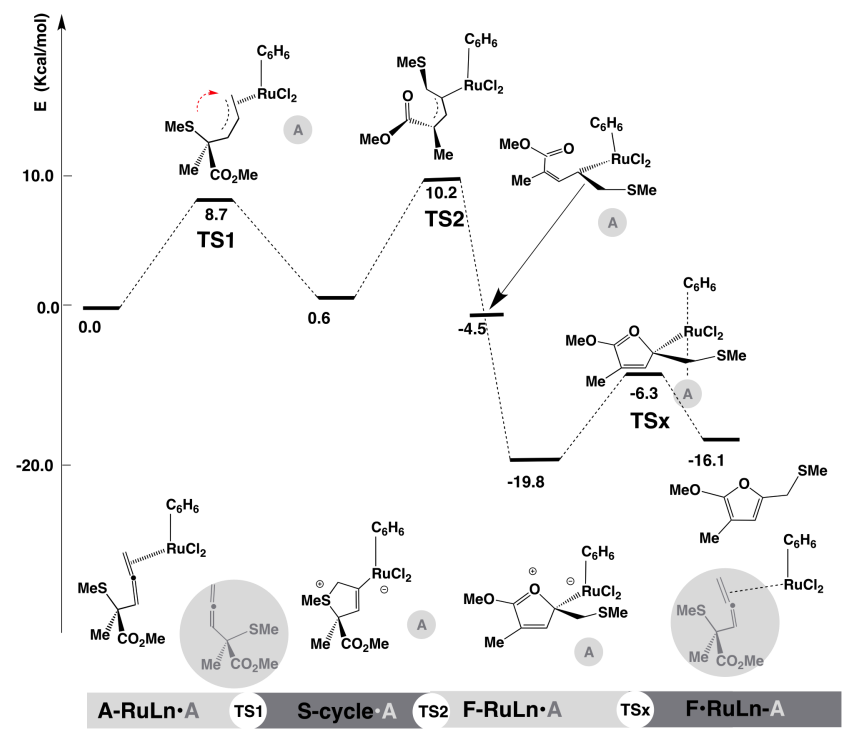

Fig. 6. Energy profile (in kcal.mol ${ }^{-1}$ ) for the $\mathrm{RuCl}_{2} \bullet\left\{\mathrm{C}_{6} \mathrm{H}_{6}\right\}$ catalyst, at the M06-D3/lanl2dz(d) level. ${ }^{A}$ represents the additional allene molecule.

\subsection{Discussion}

Fig 7 summarizes our results for the three catalysts studied here. The first step (S-Cycle formation, through TS1) cannot be a limiting step because the barriere is always small. Gold and Platinium have higher barriers than Ruthenium and it is consistent that their yields are smaller in the experimental results. Our study shows also that the limiting steps are different for Gold and Platinium. In the Gold catalyst the furane formation has a high barrier, but the catalyst regeneration is easy, while the reverse is observed for platinium.

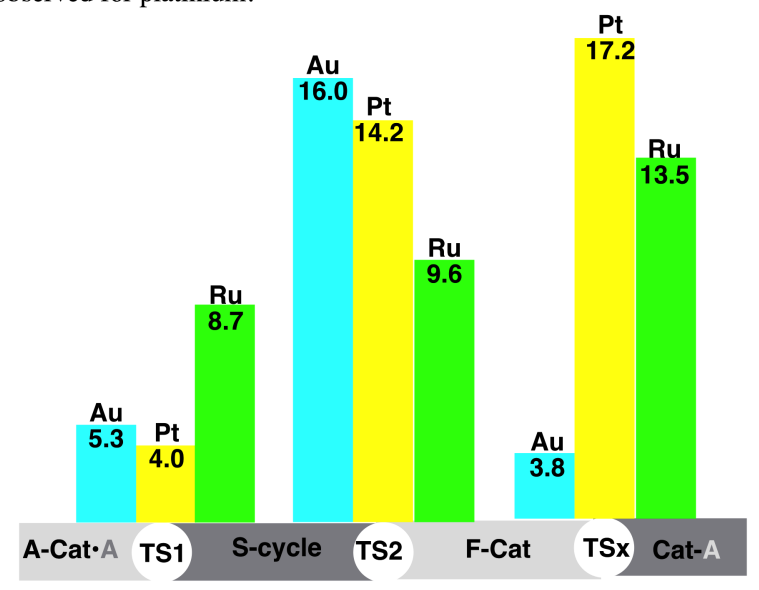

Fig. 7. Barriers of the different steps for the three catalysts (in kcal.mol ${ }^{-1}$ ).

It shall be noted that the mechanism initially proposed fits with the computational results, except for the carbenoid intermediate, which could not be found as a minimum. The computations also provide NBO charges[62,63] on the atoms and those can be used to better clarify the way we write mechanisms. For the key intermediates of the Ru catalytic cycle, the sum of charges on fragments are provided below (Fig 8) and details are in Table 2. It is shown that from RuLn-A to RuLn-Cycle-S, the charge on sulfur atom gets more positive (from +0.14 to +0.65 ). This charge returns to normal in the product RuLn-F. The modification of the charge is consistent with the written mechanism.

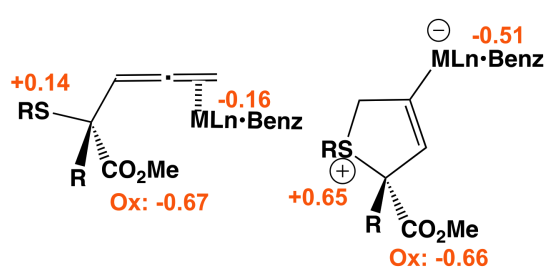

RuLn-A RuLn-Cycle-S

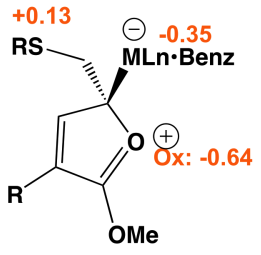

RuLn-F
Fig. 8. NBO charges (orange) on $\mathrm{S}$, on the active Oxygen atom (Ox) and on the $\mathrm{RuCl}_{2}$ •benzene catalyst (as the sum of the atomic charges per fragment).

On the catalyst fragment RuLn (which includes benzene) the variation of the charge is also consistent with the charge written in the mechanism: upon the Metal - Carbon bond formation, from RuLn-A to RuLn-Cycle$\mathbf{S}$, the charge goes from -0.16 to -0.51 , then -0.35 in RuLn-F. Such a negative charge on the catalyst does not mean that the metal bears a negative charge: the charge variation is transmitted to the ligands. Hence, 
the charge on the metal is fairly constant, and of course positive $(+0.20$, +0.22 , then +0.25 ) while the two chlorines are negatively charged in the three intermediates $(-0.90,-1.08$, then -1.06$)$. The total charge on the benzene is also modified along the reaction path $(+0.53,+0.35$, then $+0.46)$.

Table 2: Details of the NBO charges on the Ru catalyst.

\begin{tabular}{ccccl}
\hline Intermediate & Total & $\mathrm{Ru}$ & Benzene & Chlorines \\
\hline RuLn-A & -0.16 & +0.20 & +0.53 & -0.90 \\
RuLn-Cycle-S & -0.51 & +0.22 & +0.35 & -1.08 \\
RuLn-F -F & -0.35 & +0.25 & +0.46 & -1.06 \\
\hline
\end{tabular}

\section{Conclusions}

The computations finally confirmed the initially proposed mechanism, with two major clarifications: one is that the gold catalyst in the experiment must be somehow inhibited, for instance through gold clusters. This conclusion is driven by the fact that our computations of the mechanism and the barriers we obtained for the initial gold-based catalyst are very similar to that of the other catalysts, while experimentally no reaction occurs.

Second, we showed that the Pt-based catalyst is regenerated through a stable intermediate where both the furane and the allene are in interaction with the catalyst. The rate-determining step for the $\mathrm{Ru}$ and for the $\mathrm{Pt}$ stands in this catalyst regeneration. However, the key intermediate structure is a stable intermediate in the Pt case, while it is a transition state for the Ru. Hence, we shall anticipate that for bulkier allenes the catalyst regeneration would be easier for Pt because the stable intermediate would be destabilized by the steric hindrance, while for the Ru catalyst the transition state will get higher and the catalyst regeneration would get more difficult.

\section{Acknowledgements}

Dr Yoann Coquerel is gratefully acknowledged for its continuous support and careful reading oft he manuscript. Prs Daniel Liotard and Alain Dargelos are acknowledged for the CHAIN program and the coupling between Gaussian 09 and AMPAC 10. The coupling of the two codes helped us to find the different reactions paths.

\section{References}

[1] essor N. Krause, essor A.S.K. Hashmi, eds., Modern Allene Chemistry, Wiley-VCH Verlag GmbH, 2004.

doi:10.1002/9783527619573.fmatter.

[2] S. Ma, Palladium-Catalyzed Two- or Three-Component Cyclization of Functionalized Allenes, SpringerLink. (n.d.) 183210. doi:10.1007/b104131.

[3] L. Brandsma, N. Nedolya A., Synthesis. (2004) 735.

[4] S. Ma, Some Typical Advances in the Synthetic Applications of Allenes, Chem. Rev. 105 (2005) 2829-2872. doi:10.1021/cr020024j.

[5] S. Ma, Recent Advances in the chemistry of allenes, Aldrichimica Acta. 40 (2004) 91-102.

http://www.sigmaaldrich.com/ifb/acta/v40/acta-vol402007.html\#111/z (accessed September 12, 2017).

[6] H. Kim, L.J. Williams, Recent developments in allenebased synthetic methods, Curr. Opin. Drug Discov. Devel. 11 (2008) 870-894.
[7] M. Brasholz, H.-U. Reissig, R. Zimmer, Sugars, Alkaloids, and Heteroaromatics: Exploring Heterocyclic Chemistry with Alkoxyallenes, Acc. Chem. Res. 42 (2009) 45-56. doi:10.1021/ar800011h.

[8] S. Ma, Electrophilic Addition and Cyclization Reactions of Allenes, Acc. Chem. Res. 42 (2009) 1679-1688.

doi:10.1021/ar900153r.

[9] C. Aubert, L. Fensterbank, P. Garcia, M. Malacria, A. Simonneau, Transition Metal Catalyzed Cycloisomerizations of 1,n-Allenynes and -Allenenes, Chem. Rev. 111 (2011) 1954-1993. doi:10.1021/cr100376w.

[10] S. Yu, S. Ma, Allenes in Catalytic Asymmetric Synthesis and Natural Product Syntheses, Angew. Chem. Int. Ed. 51 (2012) 3074-3112. doi:10.1002/anie.201101460.

[11] J. Ye, S. Ma, Palladium-Catalyzed Cyclization Reactions of Allenes in the Presence of Unsaturated Carbon-Carbon Bonds, Acc. Chem. Res. 47 (2014) 989-1000. doi:10.1021/ar4002069.

[12] J.A. Marshall, X.J. Wang, Synthesis of furans by silver(I)promoted cyclization of allenyl ketones and aldehydes, J. Org. Chem. 56 (1991) 960-969. doi:10.1021/jo00003a013.

[13] J.A. Marshall, G.S. Bartley, Observations Regarding the $\mathrm{Ag}(\mathrm{I})$-Catalyzed Conversion of Allenones to Furans, J. Org. Chem. 59 (1994) 7169-7171. doi:10.1021/jo00102a056.

[14] A.S.K. Hashmi, Transition Metal Catalyzed Dimerization of Allenyl Ketones, Angew. Chem. Int. Ed. Engl. 34 (1995) 15811583. doi:10.1002/anie.199515811.

[15] A.S.K. Hashmi, T.L. Ruppert, T. Knöfel, J.W. Bats, C-CBond Formation by the Palladium-Catalyzed

Cycloisomerization/Dimerization of Terminal Allenyl Ketones: Selectivity and Mechanistic Aspects, J. Org. Chem. 62 (1997) 7295-7304. doi:10.1021/jo970837l.

[16] A.S.K. Hashmi, L. Schwarz, J.-H. Choi, T.M. Frost, A New Gold-Catalyzed C-C Bond Formation, Angew. Chem. Int. Ed. 39 (2000) 2285-2288. doi:10.1002/1521-

3773(20000703)39:13<2285::AID-ANIE2285>3.0.CO;2-F.

[17] S. Ma, J. Zhang, L. Lu, Pd0-Catalyzed Coupling Cyclization Reaction of Aryl or 1Alkenyl Halides with 1,2-Allenyl Ketones: Scope and Mechanism. An Efficient Assembly of 2,3,4-, 2,3,5-Tri- and 2,3,4,5-Tetrasubstituted Furans, Chem. - Eur. J. 9 (2003) 2447-2456. doi:10.1002/chem.200204664.

[18] A.V. Gulevich, A.S. Dudnik, N. Chernyak, V. Gevorgyan, Transition Metal-Mediated Synthesis of Monocyclic Aromatic Heterocycles, Chem. Rev. 113 (2013) 3084-3213. doi:10.1021/cr300333u.

[19] W.J. Moran, A. Rodríguez, Metal-catalyzed Furan Synthesis. A Review, Org. Prep. Proced. Int. 44 (2012) 103-130. doi:10.1080/00304948.2012.657558.

[20] S.P. Morcillo, D. Leboeuf, C. Bour, V. Gandon, CalciumCatalyzed Synthesis of Polysubstituted 2-Alkenylfurans from $\beta$ Keto Esters Tethered to Propargyl Alcohols, Chem. - Eur. J. 22 (2016) 16974-16978. doi:10.1002/chem.201603929.

[21] A.S. Dudnik, A.W. Sromek, M. Rubina, J.T. Kim, A.V. Kel'i, V. Gevorgyan, Metal-Catalyzed 1,2-Shift of Diverse Migrating Groups in Allenyl Systems as a New Paradigm toward Densely Functionalized Heterocycles, J. Am. Chem. Soc. 130 (2008) 1440-1452. doi:10.1021/ja0773507.

[22] C. Bruneau, P.H. Dixneuf, Metal Vinylidenes and Allenylidenes in Catalysis: Applications in Anti-Markovnikov Additions to Terminal Alkynes and Alkene Metathesis, Angew. Chem. Int. Ed. 45 (2006) 2176-2203. doi:10.1002/anie.200501391. 
[23] J.T. Kim, A.V. Kel'in, V. Gevorgyan, 1,2-Migration of the thio group in allenyl sulfides: efficient synthesis of 3-thiosubstituted furans and pyrroles, Angew. Chem. Int. Ed Engl. 42 (2003) 98-101.

[24] X.-L. Hou, Z. Yang, H.N.C. Wong, Chapter 5.3 Fivemembered ring systems: Furans and benzofurans, in: G.W.G. and J.A. Joule (Ed.), Prog. Heterocycl. Chem., Elsevier, 2003: pp. 167205.

http://www.sciencedirect.com/science/article/pii/S0959638003 800107 (accessed January 6, 2017).

[25] B.H. Lipshutz, Five-membered heteroaromatic rings as intermediates in organic synthesis, Chem. Rev. 86 (1986) 795819. doi:10.1021/cr00075a005.

[26] A. Arcadi, Gold-catalyzed synthesis of heterocycles, in: Gold Catal., IMPERIAL COLLEGE PRESS, 2011: pp. 175-224.

http://www.worldscientific.com/doi/abs/10.1142/97818481685 34_0005 (accessed January 6, 2017).

[27] A. Aponick, C.-Y. Li, J. Malinge, E.F. Marques, An Extremely Facile Synthesis of Furans, Pyrroles, and Thiophenes by the Dehydrative Cyclization of Propargyl Alcohols, Org. Lett. 11 (2009) 4624-4627. doi:10.1021/ol901901m.

[28] Z.-W. Chen, M.-T. Luo, Y.-L. Wen, M. Ye, Z.-G. Zhou, L.$X$. Liu, A Highly Efficient Synthesis of 2,5-Disubstituted Furans from Enyne Acetates Catalyzed by Lewis Acid and Palladium, Synlett. 25 (2014) 2341-2344. doi:10.1055/s-0034-1379213.

[29] S. Hosseyni, Y. Su, X. Shi, Gold Catalyzed Synthesis of Substituted Furan by Intermolecular Cascade Reaction of Propargyl Alcohol and Alkyne, Org. Lett. 17 (2015) 6010-6013. doi:10.1021/acs.orglett.5b02980.

[30] Y. Liu, F. Song, Z. Song, M. Liu, B. Yan, Gold-Catalyzed Cyclization of (Z)-2-En-4-yn-1-ols: Highly Efficient Synthesis of Fully Substituted Dihydrofurans and Furans, Org. Lett. 7 (2005) 5409-5412. doi:10.1021/ol052160r.

[31] R.K. Shiroodi, C.I.R. Vera, A.S. Dudnik, V. Gevorgyan, Synthesis of furans and pyrroles via migratory and double migratory cycloisomerization reactions of homopropargylic aldehydes and imines, Tetrahedron Lett. 56 (2015) 3251-3254. doi:10.1016/j.tetlet.2015.01.006.

[32] G. Zhou, F. Liu, J. Zhang, Enantioselective GoldCatalyzed Functionalization of Unreactive sp3 C-H Bonds through a Redox-Neutral Domino Reaction, Chem. - Eur. J. 17 (2011) 3101-3104. doi:10.1002/chem.201100019.

[33] S.J. Pridmore, P.A. Slatford, J.E. Taylor, M.K. Whittlesey, J.M.J. Williams, Synthesis of furans, pyrroles and pyridazines by a ruthenium-catalysed isomerisation of alkynediols and in situ cyclisation, Tetrahedron. 65 (2009) 89818986. doi:10.1016/j.tet.2009.06.108.

[34] V. Cadierno, J. Gimeno, N. Nebra, A Novel Propargylation/Cycloisomerization Tandem Process Catalyzed by a Ruthenium(II)/Trifluoroacetic Acid System: One-Pot Entry to Fully Substituted Furans from Readily Available Secondary Propargylic Alcohols and 1,3-Dicarbonyl Compounds, Adv. Synth. Catal. 349 (2007) 382-394. doi:10.1002/adsc.200600366.

[35] L. Peng, X. Zhang, M. Ma, J. Wang, Transition-MetalCatalyzed Rearrangement of Allenyl Sulfides: A Route to Furan Derivatives, Angew. Chem. Int. Ed. 46 (2007) 1905-1908. doi:10.1002/anie.200604299.

[36] M. Frisch, G. Trucks, H. Schlegel, G. Scuseria, M. Robb, J. Cheeseman, G. Scalmani, V. Barone, B. Mennucci, G.

Petersson, H. Nakatsuji, M. Caricato, X. Li, H. Hratchian, A. Izmaylov, J. Bloino, G. Zheng, J. Sonnenberg, M. Hada, M. Ehara,
K. Toyota, R. Fukuda, J. Hasegawa, M. Ishida, T. Nakajima, Y. Honda, O. Kitao, H. Nakai, T. Vreven, J. Montgomery, J. Peralta, F. Ogliaro, M. Bearpark, J. Heyd, E. Brothers, K. Kudin, V. Staroverov, R. Kobayashi, J. Normand, K. Raghavachari, A. Rendell, J. Burant, S. Iyengar, J. Tomasi, M. Cossi, N. Rega, J. Millam, M. Klene, J. Knox, J. Cross, V. Bakken, C. Adamo, J. Jaramillo, R. Gomperts, R. Stratmann, O. Yazyev, A. Austin, R. Cammi, C. Pomelli, J. Ochterski, R. Martin, K. Morokuma, V. Zakrzewski, G. Voth, P. Salvador, J. Dannenberg, S. Dapprich, A. Daniels, Farkas, J. Foresman, J. Ortiz, J. Cioslowski, D. Fox, Gaussian 09, Revision D.01, Gaussian 09 Revis. D01 Gaussian Inc Wallingford CT. (2013).

[37] G. Scalmani, M.J. Frisch, Continuous surface charge polarizable continuum models of solvation. I. General formalism, J. Chem. Phys. 132 (2010) 114110. doi:10.1063/1.3359469.

[38] Y. Zhao, D.G. Truhlar, The M06 suite of density functionals for main group thermochemistry, thermochemical kinetics, noncovalent interactions, excited states, and transition elements: two new functionals and systematic testing of four M06-class functionals and 12 other functionals, Theor. Chem Acc. 120 (2008) 215-241. doi:10.1007/s00214-007-0310-x.

[39] N. Marom, A. Tkatchenko, M. Rossi, V.V. Gobre, O. Hod, M. Scheffler, L. Kronik, Dispersion Interactions with DensityFunctional Theory: Benchmarking Semiempirical and Interatomic Pairwise Corrected Density Functionals, J. Chem. Theory Comput. 7 (2011) 3944-3951. doi:10.1021/ct2005616.

[40] Y. Minenkov, Å. Singstad, G. Occhipinti, V.R. Jensen, The accuracy of DFT-optimized geometries of functional transition metal compounds: a validation study of catalysts for olefin metathesis and other reactions in the homogeneous phase, Dalton Trans. 41 (2012) 5526-5541. doi:10.1039/C2DT12232D.

[41] S. Grimme, J. Antony, S. Ehrlich, H. Krieg, A consistent and accurate ab initio parametrization of density functional dispersion correction (DFT-D) for the 94 elements H-Pu, J. Chem. Phys. 132 (2010) 154104. doi:10.1063/1.3382344.

[42] S. Grimme, Density functional theory with London dispersion corrections, Wiley Interdiscip. Rev. Comput. Mol. Sci. 1 (2011) 211-228. doi:10.1002/wcms.30.

[43] J. Řezáč, P. Hobza, Advanced Corrections of Hydrogen Bonding and Dispersion for Semiempirical Quantum Mechanical Methods, J. Chem. Theory Comput. 8 (2012) 141-151. doi:10.1021/ct200751e.

[44] S. Rösel, H. Quanz, C. Logemann, J. Becker, E. Mossou, L. Cañadillas-Delgado, E. Caldeweyher, S. Grimme, P.R. Schreiner, London Dispersion Enables the Shortest Intermolecular Hydrocarbon H...H Contact, J. Am. Chem. Soc. 139 (2017) 74287431. doi:10.1021/jacs.7b01879.

[45] D.J. Gorin, F.D. Toste, Relativistic effects in homogeneous gold catalysis, Nature. 446 (2007) 395-403. doi:10.1038/nature05592.

[46] D. Liotard, J.-P. Penot, Critical Paths and Passes: Application to Quantum Chemistry, in: Numer. Methods Study Crit. Phenom., Springer, Berlin, Heidelberg, 1981: pp. 213-221. doi:10.1007/978-3-642-81703-8_27.

[47] D.A. Liotard, Algorithmic tools in the study of semiempirical potential surfaces, Int. J. Quantum Chem. 44 (1992) 723-741. doi:10.1002/qua.560440505.

[48] M. J. S. Dewar, A. J. Holder, R. D. Dennington, D. A. Liotard, D. G.Truhlar, T. A. Keith, J. M. Millam, C. D. Harris. Holder, Ampac 10, Semichem, Inc., (n.d.). 
[49] K. Ando, Mechanistic Study on the Gold-Catalyzed C-S Bond Formation of $\alpha$-Thioallenes To Form 2,5-

Dihydrothiophenes, J. Org. Chem. 75 (2010) 8516-8521. doi:10.1021/jo101883j.

[50] A.S. Dudnik, Y. Xia, Y. Li, V. Gevorgyan, ComputationGuided Development of Au-Catalyzed Cycloisomerizations Proceeding via 1,2-Si or 1,2-H Migrations: Regiodivergent Synthesis of Silylfurans, J. Am. Chem. Soc. 132 (2010) 7645-7655. doi:10.1021/ja910290c.

[51] L. Fensterbank, M. Malacria, Molecular Complexity from Polyunsaturated Substrates: The Gold Catalysis Approach, Acc. Chem. Res. 47 (2014) 953-965. doi:10.1021/ar4002334.

[52] V. Gandon, G. Lemière, A. Hours, L. Fensterbank, M. Malacria, The role of bent acyclic allene gold complexes in axisto-center chirality transfers, Angew. Chem. Int. Ed Engl. 47 (2008) 7534-7538. doi:10.1002/anie.200802332.

[53] R.S. Paton, F. Maseras, Gold(I)-Catalyzed Intermolecular Hydroalkoxylation of Allenes: A DFT study, Org. Lett. 11 (2009) 2237-2240. doi:10.1021/ol9004646.

[54] W. Yang, A.S.K. Hashmi, Mechanistic insights into the gold chemistry of allenes, Chem. Soc. Rev. 43 (2014) 2941-2955. doi:10.1039/C3CS60441A.

[55] J.E. Bailie, G.J. Hutchings, Promotion by sulfur of gold catalysts for crotyl alcohol formation from crotonaldehyde hydrogenation, Chem. Commun. (1999) 2151-2152. doi:10.1039/A906538E.

[56] W. Debrouwer, R.A.J. Seigneur, T.S.A. Heugebaert, C.V. Stevens, Gold superacid-catalyzed preparation of benzo[c]thiophenes, Chem. Commun. 51 (2014) 729-732. doi:10.1039/C4CC07989B.
[57] Menggenbateer, M. Narsireddy, G. Ferrara, N. Nishina, T. Jin, Y. Yamamoto, Gold-catalyzed regiospecific intermolecular hydrothiolation of allenes, Tetrahedron Lett. 51 (2010) 46274629. doi:10.1016/j.tetlet.2010.06.125.

[58] N. Morita, N. Krause, The First Gold-Catalyzed C-S Bond Formation: Cycloisomerization of $\alpha$-Thioallenes to 2,5-

Dihydrothiophenes, Angew. Chem. Int. Ed. 45 (2006) 1897-1899. doi:10.1002/anie.200503846.

[59] I. Nakamura, T. Sato, Y. Yamamoto, Gold-Catalyzed Intramolecular Carbothiolation of Alkynes: Synthesis of 2,3Disubstituted Benzothiophenes from ( $\alpha$-Alkoxy Alkyl) (orthoAlkynyl Phenyl) Sulfides, Angew. Chem. Int. Ed. 45 (2006) 44734475. doi:10.1002/anie.200601178.

[60] L. Peng, X. Zhang, S. Zhang, J. Wang, Au-Catalyzed Reaction of Propargylic Sulfides and Dithioacetals, J. Org. Chem. 72 (2007) 1192-1197. doi:10.1021/jo0618674.

[61] J.R. Reimers, M.J. Ford, A. Halder, J. Ulstrup, N.S. Hush, Gold surfaces and nanoparticles are protected by $\mathrm{Au}(0)$-thiyl species and are destroyed when $\mathrm{Au}(\mathrm{I})$-thiolates form, Proc. Natl. Acad. Sci. U. S. A. 113 (2016) E1424-E1433. doi:10.1073/pnas.1600472113.

[62] E.D. Glendening, C.R. Landis, F. Weinhold, NBO 6.0: Natural bond orbital analysis program, J. Comput. Chem. 34 (2013) 1429-1437.

[63] E.D. Glendening, C.R. Landis, F. Weinhold, Natural bond orbital methods, Wiley Interdiscip. Rev. Comput. Mol. Sci. 2 (2012) 1-42. 


\section{Metal-Catalysed Rearrangement of Allenylsulfides to}

\section{Furan: A Theoretical Mechanistic Approach}

Michel Rajzmann, ${ }^{*[a]}$ Stephane Humbel, ${ }^{[[a]}$ Jianbo Wang ${ }^{[b]}$

[a] Dr M. Rajzmann, Pr S. Humbel : Aix Marseille Univ, CNRS, Centrale Marseille, iSm2, Marseille, France

[b] Pr J. Wang: Key Laboratory of Bioorganic Chemistry and Molecular Engineering of Ministry of Education, College of Chemistry, Peking University, Beijing 100871, China

\section{Endo orientation of the MLn-A complex}

The metal can bind on either side of the allene bond in a low barrier process (about $+3 \mathrm{kcal} / \mathrm{mol}$ ). Only the exo (or Trans) orientation can directly lead to the product.

The endo orientation is slightly higher in energy than the exo when Au or Pt are used as catalysts. Only for $\mathrm{Ru}$, is the first endo (or $\mathrm{Cis}$ ) intermediate lower in energy than the exo (Trans). But only the exo (Trans) can lead to the formation of the Furane, and this is more exothermic. Bellow are details about the endo coordination and various subsequent intermediates.
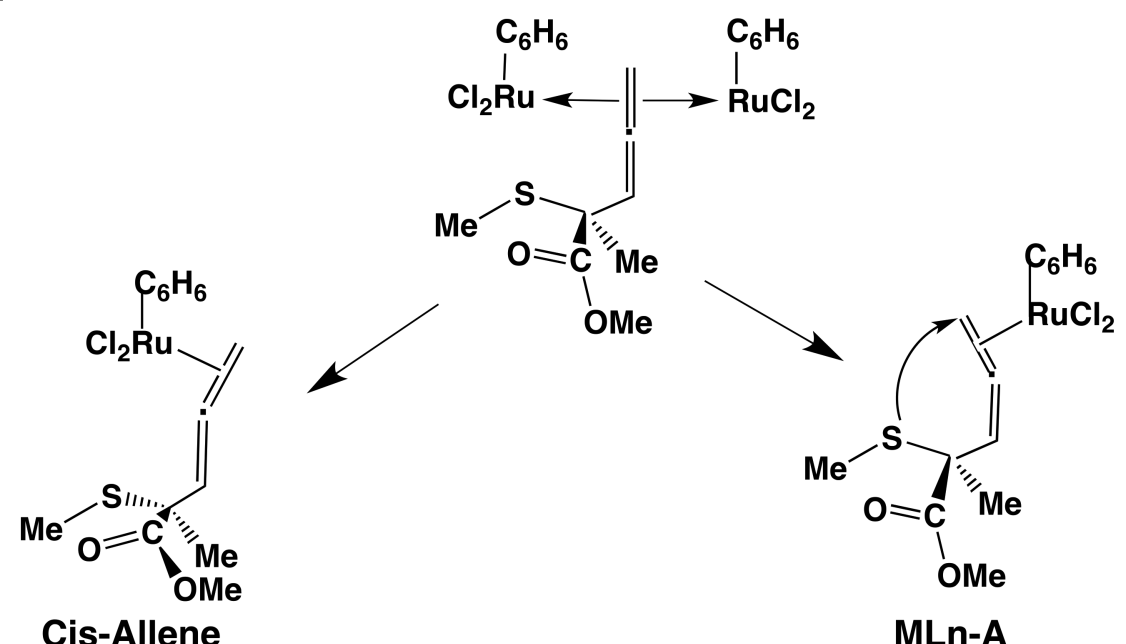

Cis-Allene

$$
\text { MLn-A }
$$

$$
E=-2.86
$$

$E=0.00$

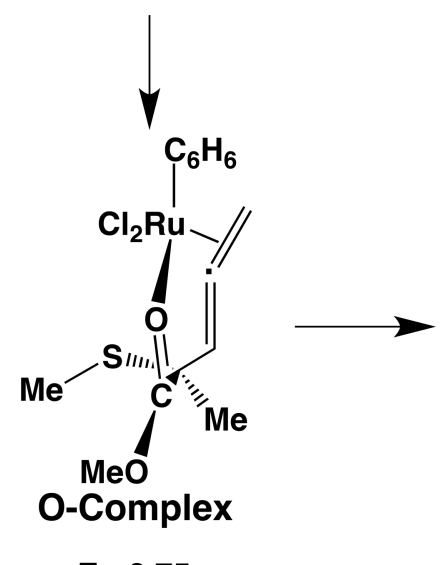

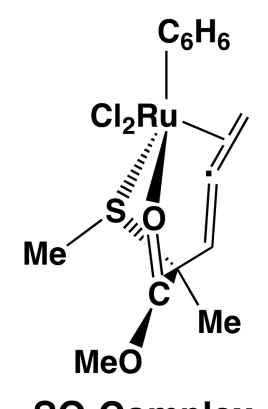

SO-Complex<smiles>CCCCCCCC[C@@H](CCCC)c1cc(C)c(OC)o1</smiles>

$E=-0.75$

$E=-8.90$

$E=-19.81$

Scheme S1: Structures \& energies for $\mathbf{R u C l}_{2}\left(\mathrm{C}_{6} \mathrm{H}_{6}\right)$-A. Reference of the energies is that of $\mathrm{RuCl}_{2}\left(\mathrm{C}_{6} \mathrm{H}_{6}\right)-\mathrm{A}(\mathrm{MLn}-\mathrm{A}) 0.00 \mathrm{kcal} . \mathrm{mol}^{-1}$. 
Figure S1 shows the main intermediates and Table S1 collects NBO charges (NBO version 6). ${ }^{1}$ The Ru-benzene distance increases from $2.20 \AA$ (in MIn-F) up to $2.71 \AA$ in the SOcomplex (Figure 1). The hapticity is also modified $\eta 6$ in $\mathbf{M l n}-\mathbf{F}$, against $\eta 2$ in the SOcomplex. The charges (Table 1) shows very well the modification of the role of the benzene moieties: from strongly donating - MLn-F shows very positive charges on the benzene up moderate donnor when the benzene is not close to the metal (as in the SO and O complexes).

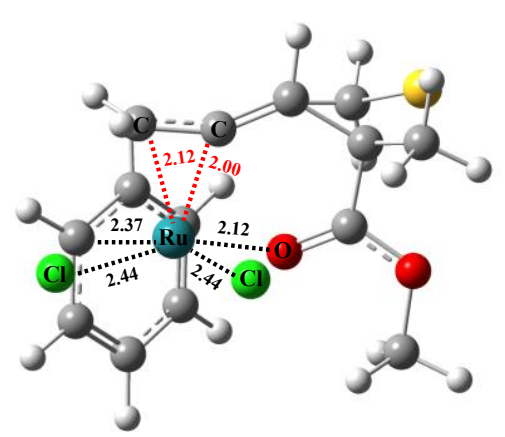

(a)

(b)

(c)
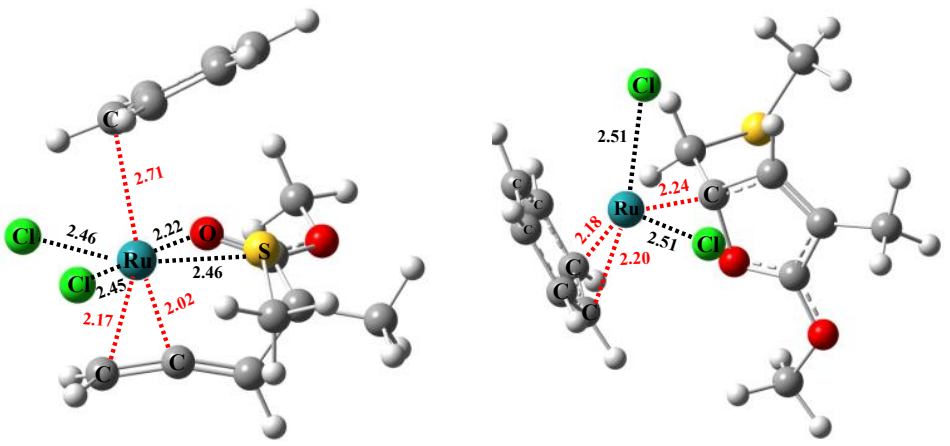

Figure S1: Main intermediates (a) O-Complex, (b) SO-Complex, (c) MLn-F.

Table S1: NBO6 Total charge for Ru, O (carbonyl), S, and groups of atoms : the $2 \mathrm{Cl}$ atoms, and benzene molecule.

\begin{tabular}{|c|c|c|c|c|c|}
\hline $\begin{array}{c}\text { Total } \\
\text { Charge }\end{array}$ & MLn-A & S-Cycle & MLn-F & O-Complex & SO-Complex \\
\hline Ru & 0.205 & 0.222 & 0.246 & 0.525 & 0.361 \\
\hline O & -0.673 & -0.657 & -0.639 & -0.663 & -0.637 \\
\hline S & 0.139 & 0.649 & 0.133 & 0.151 & 0.361 \\
\hline Cl2 & -0.896 & -1.085 & -1.057 & -0.908 & -1.001 \\
\hline Benzene & 0.534 & 0.351 & 0.463 & 0.147 & 0.137 \\
\hline
\end{tabular}

To make the Trans complex from the Cis can be achieved with a new Allene molecule. The barriere is $12.8 \mathrm{kcal}^{\mathrm{mol}}{ }^{-1}$ (Figure S2). This is much easier than rotating around the double bond ( 3 step process with the highest at $34.6 \mathrm{kcal}_{\mathrm{mol}}{ }^{-1}$.

The complete path from the SO-complex to MLn-F is given in Figure S2 bellow. The equilibrium constant between the SO complex and the RuLn-F product corresponds at $80^{\circ} \mathrm{C}$, gives a ratio SO complex/RuLn-F of about $10^{-7}$, and is smaller at $25^{\circ} \mathrm{C}\left(\mathrm{k}_{\mathrm{d}} / \mathrm{k}_{\mathrm{f}} \sim 10^{-8}\right)$. Hence, under thermodynamic conditions, the Cis-Allene, readily transforms to the furane product. 


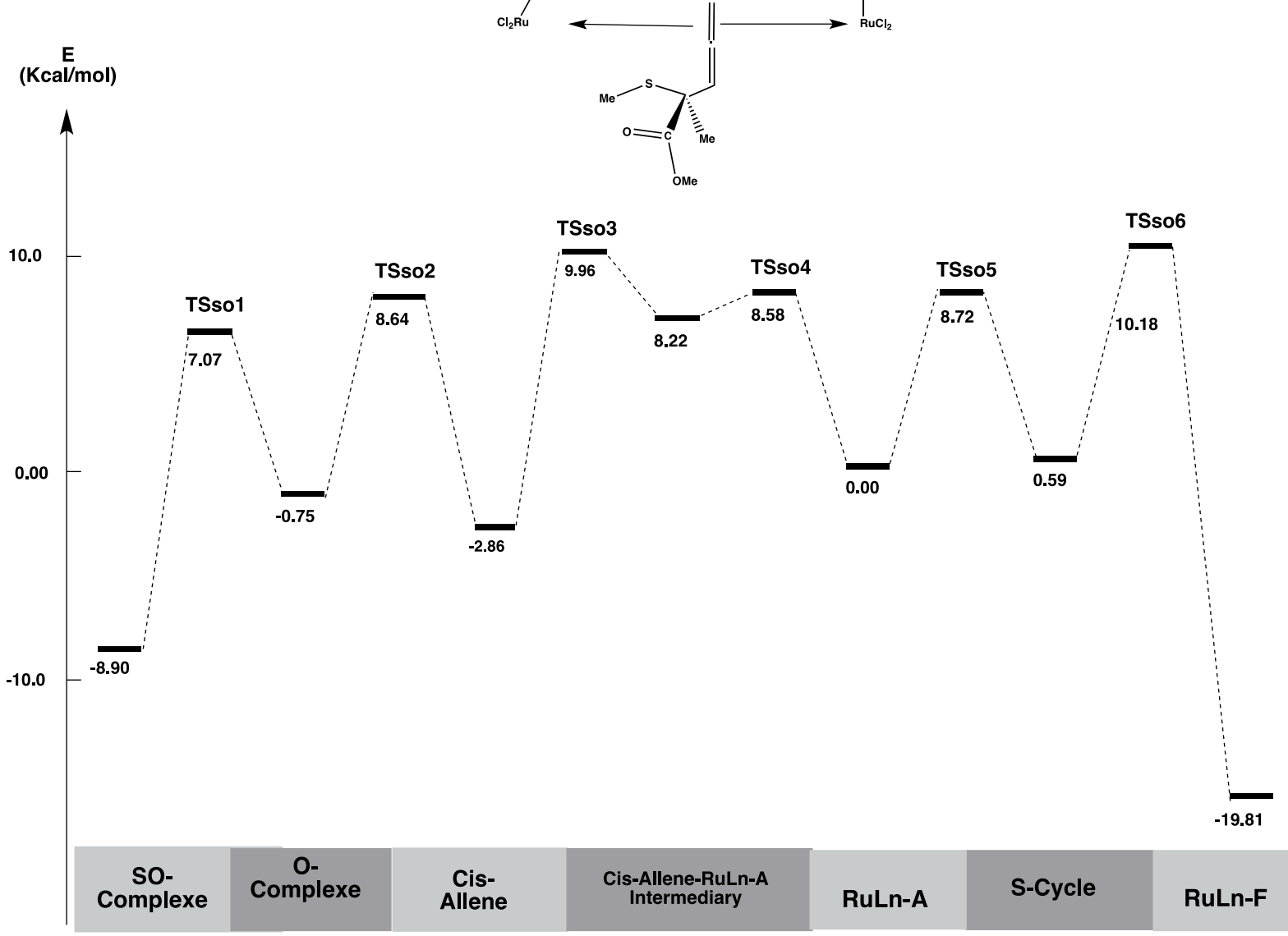

Figure S2: Energy profile for O-Complex \& SO-Complex formation.

Table S2: Energies for O-Complex, So-Complex etc... paths (Energy in ua, $\Delta E$ in kcal.mol ${ }^{-1}$ ).

\begin{tabular}{|c|c|c|}
\hline & Energy & $\Delta \mathrm{E}$ \\
\hline SO-Complexe & -1300.52943 & -8.90 \\
\hline TSso1 & -1300.50397 & 7.07 \\
\hline O-Complexe & -1300.51643 & -0.75 \\
\hline TSso2 & -1300.50147 & 8.64 \\
\hline Cis-Allene & -1300.51980 & -2.86 \\
\hline TSso3 & -1300.49936 & 9.96 \\
\hline Cis-Allene-MLn-A-Intermediary & -1300.50213 & 8.22 \\
\hline TSso4 & -1300.50156 & 8.58 \\
\hline RuLn-A & -1300.51524 & 0.00 \\
\hline TSso5 & -1300.50134 & 8.72 \\
\hline S-Cycle & -1300.51430 & 0.59 \\
\hline TSso6 & -1300.49902 & 10.18 \\
\hline RuLn-F & -1300.54681 & -19.81 \\
\hline
\end{tabular}

(1) Glendening, E. D.; Landis, C. R.; Weinhold, F. Wiley Interdiscip. Rev. Comput. Mol. Sci. 2012, 2 (1), 1-42. 


\section{Structures}

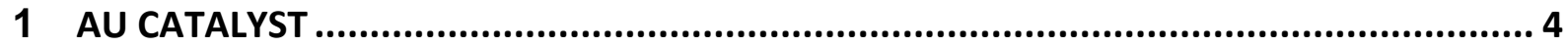

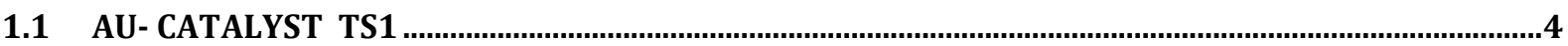

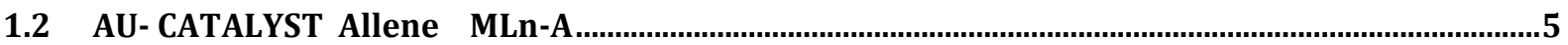

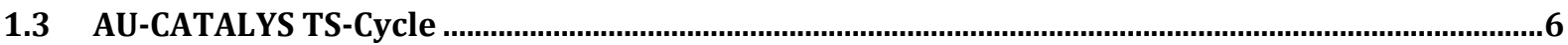

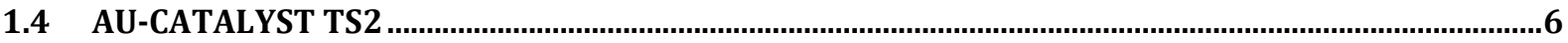

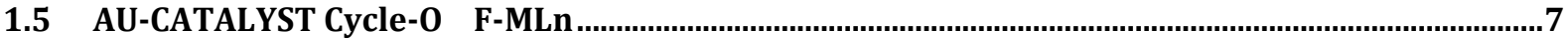

1.6 AU-REGENERATION TSX

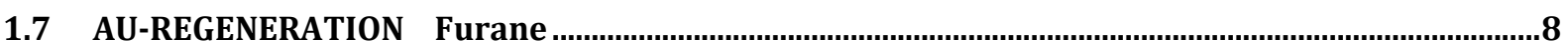

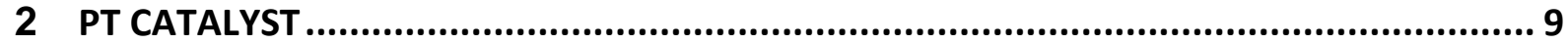

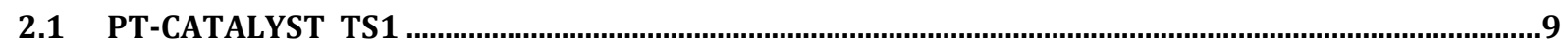

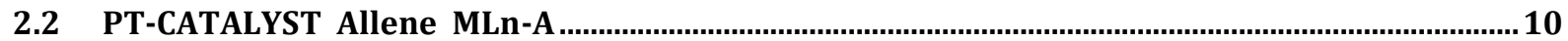

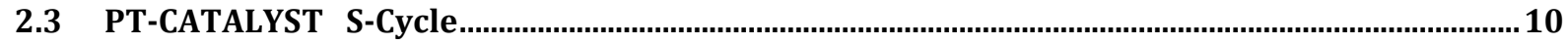

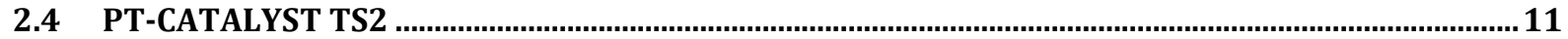

2.5 PT-CATALYST Cycle-0 F-MLn ……...............................................................................12

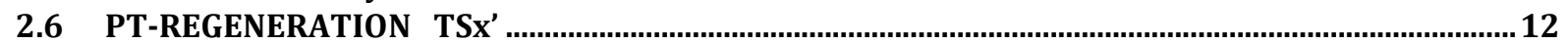

2.7 PT-REGENERATION Complexe F-MLn-A …..........................................................................13

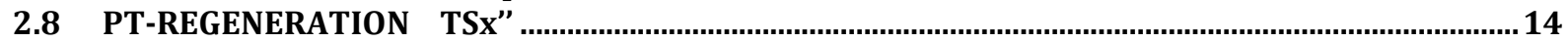

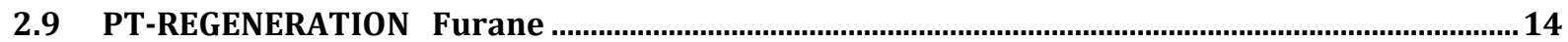

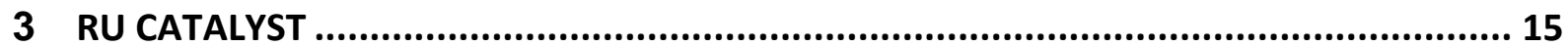

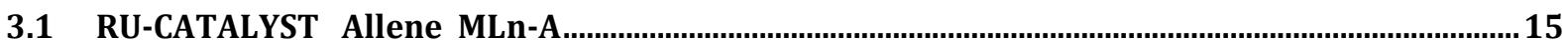

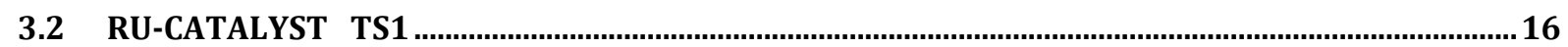

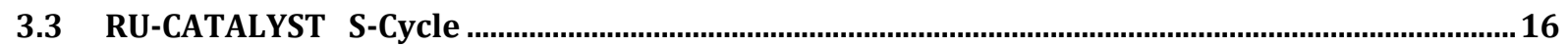

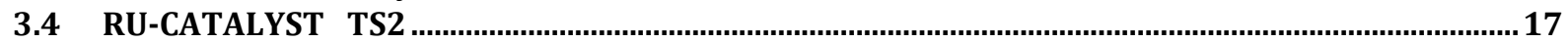

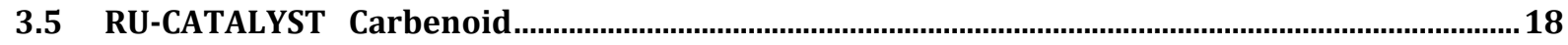

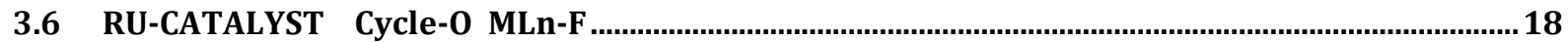

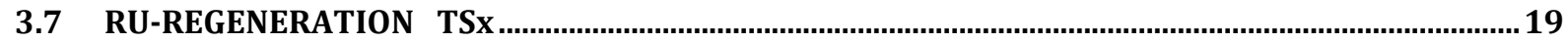

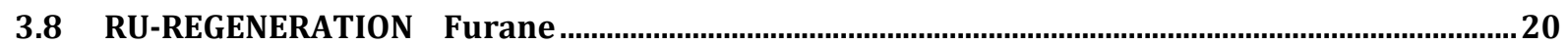

4 COORDINATION OF THE SULFUR ON THE RU CATALYST ...................................... 20

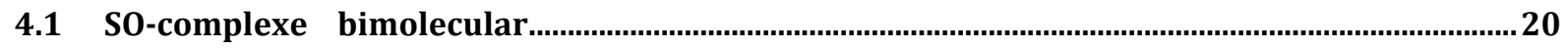

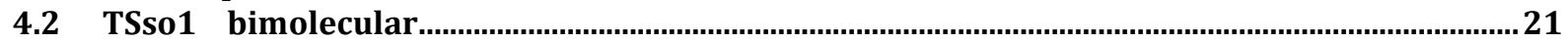

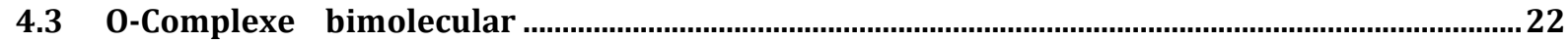

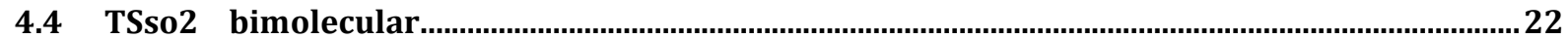

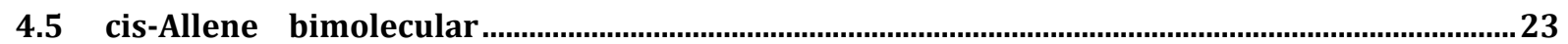

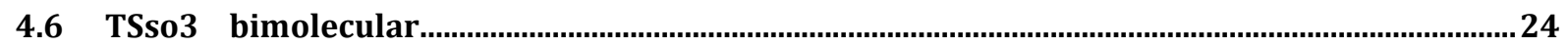

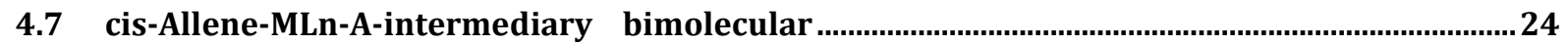

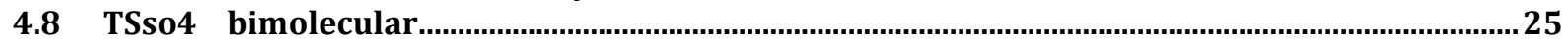

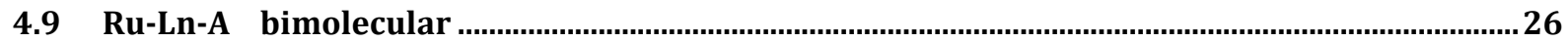

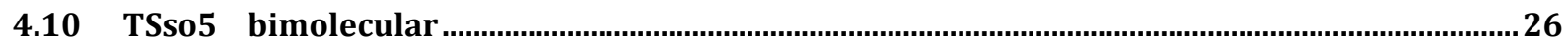

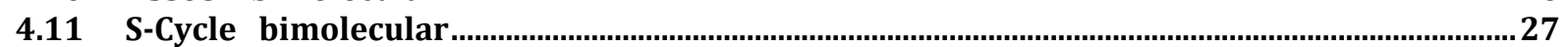

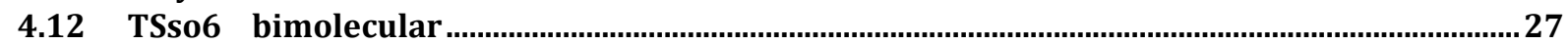

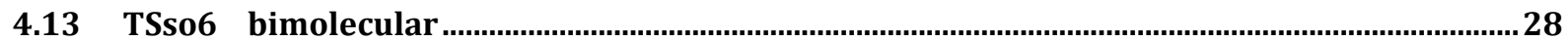

In the following, energies and xyz coordinates (in $\AA$ ) are provided for all structures. Additionally for transition states the negative frequency is provided (LOWEST FREQUENCY).

\section{Au CATALYST}

1.1 AU- CATALYST TS1

ENERGY=-1327.11544 LOWEST

FREQUENCY=-209.63 


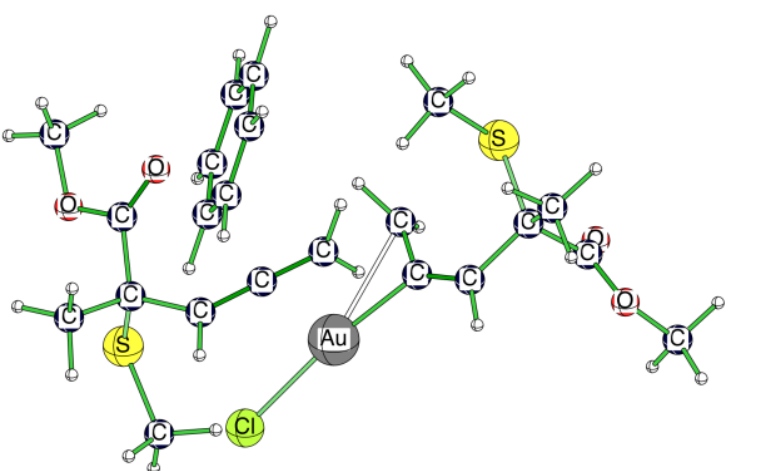

C 3.80002000

C 2.70722500

1.00712800

1.07177600

C 1.97456100

$-0.06194300$

1.01941500

C 5.07740500

$-0.20580000$

$-0.07969100$

S 3.54270300

0.26909800

0.66156100

C 1.94330600

2.29279600

$-0.29096700$

O 5.53412000

0.52672800

$-1.26915200$

O 5.56845400

0.25323900

C 6.68955000

$-0.42449700$

C 2.13967000

$-1.26152000$

$-0.45948600$

1.68894200

1.38060000

H 2.64517500

3.25848600

0.45138700

H 1.14694600

$-0.72305600$

1.88545300

H 2.52875600

25471600

$-1.45601300$

C 3.88453100

0.20496900

$-2.13252800$

H 2.50656100

1.63789600

2.45505200

H 1.72606700

3.90581500

1.25101000

H 1.37002300

(

H 7.54141300

2.57163600

$-0.35936500$

0.82224600

H 6.92736600

$-0.65320700$

1.06003900

2.30184200

H $6.42903900-1.96502500$

H 2.93409500

2.11466900

0.58352600

2.72351500

H 4.08201400

0.85666300

3.19746200

H 4.68702100

2.38199300

$\mathrm{Cl}-0.96628600$

C -0.77880200

$-3.36290800$

2.51361100

C -0.78949800

3.23533000

$-1.26916400$

C -1.18467800

1.22959500

$-3.35135200$

C -1.56097800

C -1.53178100

C -1.14981400

0.55028700

$-3.42170200$

H -0.49069500

H -0.49940700

H -1.20259000

H -1.87536300

H -1.80753100

H -1.16745200

Au 0.55817800

C -4.07695300

C -2.98316500

C -1.78387300

C -4.10550100

S -3.81898500

C -0.58381700

O -3.58699100

O -4.85376600

C -4.98350300

C -3.34750500

H -3.21416800

H 0.23985000
$1.21183500-1.02668900$

$2.55436000-0.95419300$

$4.28549400-2.07213600$

$3.10146800-4.25722700$

$0.71572500-4.38168700$

$-0.49398700-2.29866400$

$0.66239300-0.12335000$

$3.06928400 \quad 0.00773600$

$-1.57098900-0.70498200$

$0.01494200 \quad 1.72528800$

$-0.08359800 \quad 2.75381800$

$0.44985700 \quad 2.66763100$

$1.37966200 \quad 1.03415700$

$\begin{array}{ll}-1.29857000 & 0.34666500\end{array}$

$0.97498200 \quad 2.59610500$

$2.38946800 \quad 1.45741100$

$1.33512700-0.07129600$

$2.56656200-0.78276000$

$-2.75847600 \quad 1.39279400$

$-0.693117003 .63263300$

$0.43564400 \quad 2.11970800$
H -0.38556600

C -5.44098600

1.97131000

2.99392700

H -2.38310800

$-0.19917600$

H -4.12755200

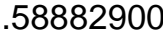

H -3.24863100

$-2.98088400$

777500

H -5.64134900

$-3.58985000$

8110100

H -5.41862000

2.35560500

2.12886600

H -4.00294000

3.33828300

0.69029000

H -5.48222400

2.90369300

$-1.62789200$

H -5.60802500

$-1.18808200$

13850700

H -6.24479400

0.55826600

$-0.13696400$

85916700

3.16412000

1.2 AU-CATALYST Allene MLn-A ENERGY=-1327.12239

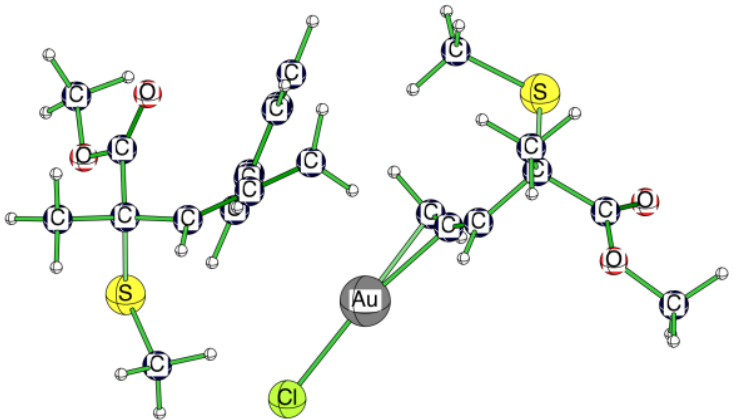

C 3.85196300

1.01284500

1.06728400

C 2.73189400

0.03661000

0.74017500

C 2.16151700

$-0.05107700$

$-0.44605300$

C 5.15629100

0.24581800

0.81954300

S 3.90791000

2.48807700

$-0.10908400$

C 1.82180300

0.19827300

$-1.74140200$

O 5.91449800

0.42746500

$-0.10391900$

O 5.31541000

$-0.69660400$

1.75361900

C 6.46385800

$-1.53533700$

1.58941900

C 2.29763200

3.29964600

0.34536200

H 2.44238300

$-0.64666100$

1.54319100

H 1.11948900

1.00179900

H 2.39633900

$-0.24660200$

$-1.98717100$

C 3.73821200

1.45553300

$-2.55446700$

H 2.35403100

H 2.12033800

3.78395400

2.52390400

H 1.47878900

4.05578200

1.32417200

H 7.37878700

2.56992400

$-0.42408300$

H 6.45554100

$-0.93410300$

0.32071000

H 6.39987200

$-2.22420400$

1.59273100

H 2.79825200

$-2.08717100$

2.43598100

H 3.75145500

1.99416400

0.64569700

H 4.57078100

0.58371600

2.69430800

Cl -0.92187700

2.11543300

3.18850200

C -0.84407600

$-3.32609300$

2.79224000

C -1.09061200

2.85769200

$-0.65122200$

C -1.63724900

2.24786000

$-2.18577000$

C -1.92561200

0.96016900

$-3.42185600$

C -1.66120400

C -1.13190200

$0.27632000-2.28935700$

$0.88150200-1.05631700$

H -0.43759700

2.17317100

$-0.99997500$

H -0.86518600

$3.86810200-2.14862100$

$2.78151000-4.34435600$

H -1.83686000

$0.49209000-4.43737500$

H -2.35683300

$-0.72507100-2.30886100$

H -1.87719200

$0.32674300-0.13945400$ 


$\begin{array}{lrrr}\text { H } & -0.97254700 & 2.64559100 & -0.02905200 \\ \text { Au } 0.56750700 & -1.45895100 & -0.91266000 \\ \text { C }-4.02298100 & 0.14053100 & 1.85699500 \\ \text { C }-2.81943300 & 0.08898000 & 2.75871600 \\ \text { C }-1.64401400 & 0.63367500 & 2.53278400 \\ \text { C }-4.07494900 & 1.42951000 & 1.03604200 \\ \text { S } & -4.00940700 & -1.34043600 & 0.63601000 \\ \text { C } & -0.46874800 & 1.18164000 & 2.33559600 \\ \text { O } & -3.58942000 & 2.48383000 & 1.38307300 \\ \text { O } & -4.80970500 & 1.27495500 & -0.06744500 \\ \text { C }-4.95087400 & 2.44022500 & -0.88117000 \\ \text { C }-3.40472200 & -2.68934100 & 1.76055900 \\ \text { H } & -2.94686200 & -0.49572700 & 3.67478200 \\ \text { H } & 0.31985600 & 0.64777500 & 1.79752200 \\ \text { H }-0.25251300 & 2.19103700 & 2.68885200 \\ \text { C }-5.30408600 & 0.09923700 & 2.69549400 \\ \text { H }-2.39344800 & -2.46991500 & 2.11470700 \\ \text { H }-4.09419300 & -2.85205200 & 2.59595700 \\ \text { H }-3.36165000 & -3.58425200 & 1.13345100 \\ \text { H } & -5.53176900 & 2.12973300 & -1.75171200 \\ \text { H }-5.47372900 & 3.23115600 & -0.33237600 \\ \text { H }-3.96519100 & 2.80708600 & -1.19159200 \\ \text { H }-5.33297700 & -0.81523600 & 3.30145300 \\ \text { H }-5.33610000 & 0.96187000 & 3.37646000 \\ \text { H } & -6.19100900 & 0.11389200 & 2.05305400\end{array}$

\subsection{AU-CATALYS TS-Cycle}

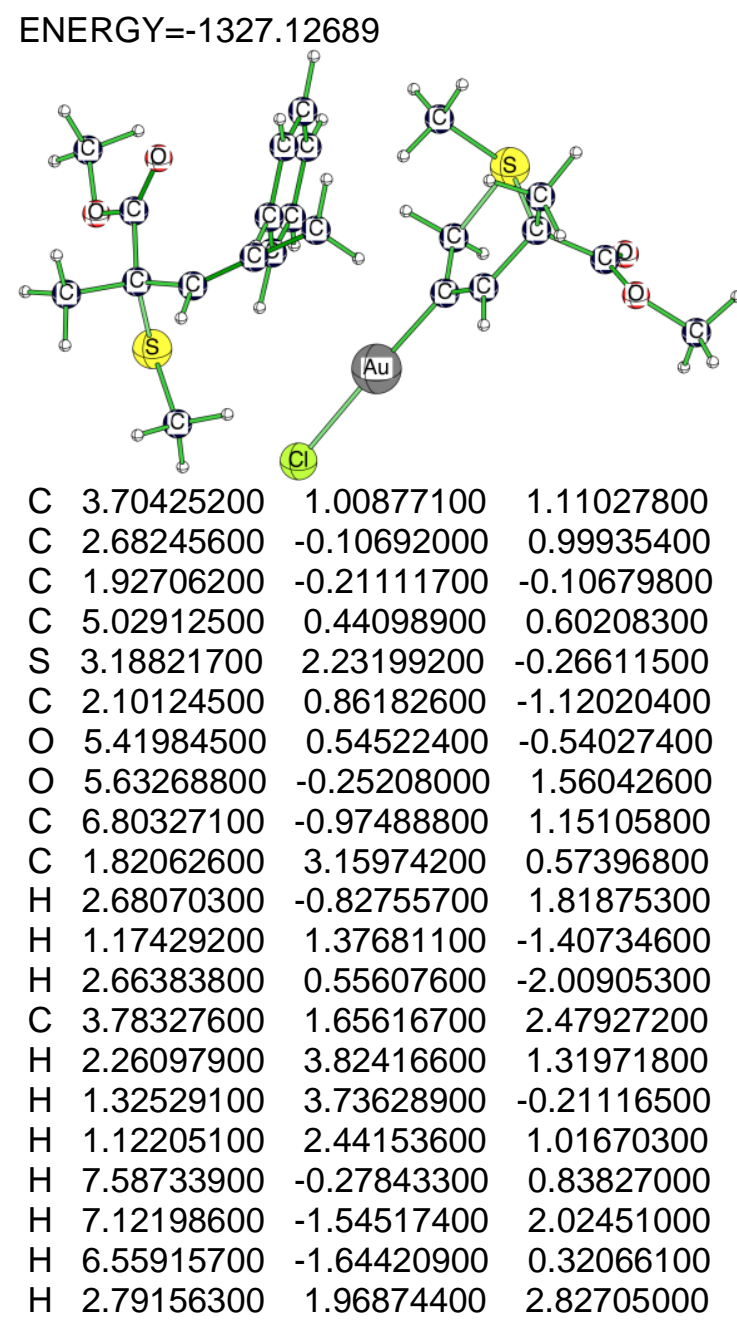
H 4.15552000
0.90435900
3.18427300
H 4.46911200
2.51132400
2.50231800
$\mathrm{Cl}-0.91100500$
$-3.46638700$
$-1.27555600$
C -0.82855700
3.30687500
$-2.15974900$
C -0.74813900
2.61602900
$-3.37386700$
C -1.04912900
C -1.42016100
C -1.47993500
C -1.19386900
1.24966600
$-3.42471700$
$0.57133100-2.25989800$
$1.25897000-1.04408700$
$2.62537800-0.99210600$
H -0.61682800
H -0.46301200
H -0.99722900
4.37604400
$-2.12658800$
$3.14553800-4.28222900$
$0.71477100-4.37181400$
H -1.66560700
H -1.74459600
H -1.27519900
Au 0.60268900
$-0.49160600$
$-2.28068900$
0.70721400
$-0.13905700$
3.15680000
$-0.04245600$
C -4.04785000
$-1.67741600$
$-0.60412100$
C -2.96258700
0.01216400
1.65319300
C -1.77232300
C -4.10313100
$-0.06922900$
2.69192800
0.48594800
2.62673700
1.38063800
0.97359900
S -3.74712800
$-1.29116700$
0.27363100
C -0.56976400
1.00900300
2.57566200
O -3.62151400
O -4.83433100
C -4.99452700
2.40098600
1.41595900
1.32703900
$-0.14241400$
2.56220700
$-0.84003800$
C -3.25910700
$-2.73984100$
1.32729200
H -3.18703200
H 0.24976800
$-0.69612000$
3.56018400
0.46074300
2.09947900
H -0.36714500
1.99436800
2.99974500
C -5.41334600
$-0.23250300$
2.30013000
H -2.29167100
H -4.03375000
$-2.55913300$
1.80519400
$-2.96111600$
2.06973900
H -3.15808600
$-3.57534400$
0.63062600
H -5.64180300
2.34296000
$-1.69118200$
H -5.45387000
H -4.02216100
3.31438800
$-0.18953500$
$2.93021900-1.18919000$
H -5.43877000
$-1.22338400$
2.77139600
H -5.60271900
H -6.21169300
0.51895100
3.08030300
$-0.18460500$

\subsection{AU-CATALYST TS2}

ENERGY $=-1327.10129 \quad$ LOWEST
FREQUENCY $=-201.60$

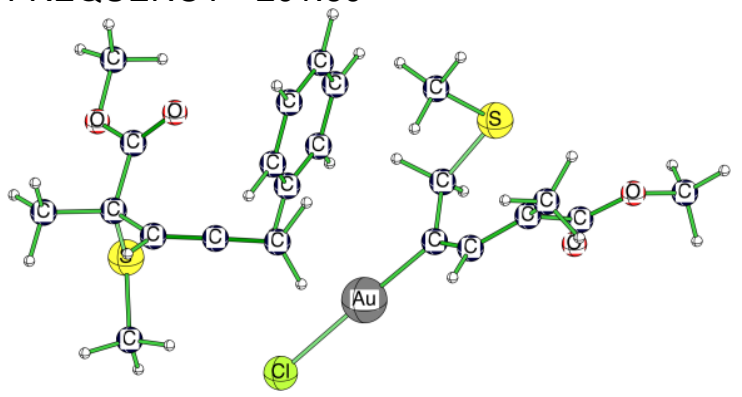

C $4.08239100 \quad 0.09545500 \quad 1.49438900$

$\begin{array}{llll}\text { C } 2.87104600 & -0.58752600 & 1.22987800\end{array}$

$\begin{array}{lll}\text { C } 2.11023300 & -0.44638200 & 0.09065400\end{array}$

C $5.18680600 \quad 0.01496700 \quad 0.46058500$

S $3.39293800 \quad 2.10821500 \quad 0.12325300$

$\begin{array}{llll}\text { C } & 2.28028500 & 0.81301800 & -0.70599600\end{array}$ 


\begin{tabular}{|c|c|c|}
\hline 5.06540400 & -0.50127400 & -0.62793700 \\
\hline 6.30969400 & 0.57152800 & 0.91716600 \\
\hline 7.39988900 & 0.58297000 & -0.01090300 \\
\hline 2.14251300 & 2.77693400 & 1.31669300 \\
\hline 2.48934900 & -1.20775900 & 2.04676100 \\
\hline 1.30756000 & 1.29991800 & -0.84849300 \\
\hline 2.71483000 & 0.61425900 & -1.69385100 \\
\hline 4.47460700 & 0.39596000 & 2.90068500 \\
\hline 2.66156900 & 3.44042300 & 2.01113900 \\
\hline 1.37679200 & 3.32093200 & 0.75621400 \\
\hline 1.68454200 & 1.93237800 & 1.84787600 \\
\hline 7.13058800 & 1.16290500 & -0.90056800 \\
\hline 8.23481300 & 1.05218700 & 0.51211600 \\
\hline 7.65657300 & -0.43782500 & -0.31136100 \\
\hline 3.59881300 & 0.42488200 & 3.55670600 \\
\hline 5.17219000 & -0.37018700 & 3.26774800 \\
\hline 5.01264400 & 1.34887300 & 2.95797600 \\
\hline-1.14625800 & -3.22155600 & -1.09838000 \\
\hline-0.30567100 & 3.32948400 & -1.58787100 \\
\hline 0.00575400 & 2.86723600 & -2.87117400 \\
\hline-0.34725100 & 1.56651200 & -3.25039200 \\
\hline-1.00781900 & 0.72824700 & -2.34682800 \\
\hline-1.30996300 & 1.18761000 & -1.06033600 \\
\hline-0.96274800 & 2.48802100 & -0.68073100 \\
\hline-0.04341000 & 4.34790500 & -1.29868200 \\
\hline 0.51673000 & 3.52170900 & -3.57628200 \\
\hline-0.10949400 & 1.20780100 & -4.25090500 \\
\hline-1.29327900 & -0.28499000 & -2.63308200 \\
\hline-1.81930400 & 0.52109500 & -0.36075300 \\
\hline-1.22660900 & 2.83812400 & 0.31833100 \\
\hline Au 0.62511900 & -1.68445500 & -0.47100000 \\
\hline-4.67940400 & 0.20354600 & 1.26062900 \\
\hline-3.83868300 & -0.33287600 & 2.38900600 \\
\hline-2.52382500 & -0.38224300 & 2.39457200 \\
\hline-4.25993000 & 1.62803100 & 0.88478600 \\
\hline-4.41166200 & -0.78318900 & -0.37220000 \\
\hline-1.21477500 & -0.45631300 & 2.37607100 \\
\hline-3.38046600 & 2.27528600 & 1.40956700 \\
\hline-5.01829700 & 2.08896200 & -0.11579400 \\
\hline-4.68084900 & 3.39206100 & -0.59272200 \\
\hline-4.33959400 & -2.52108400 & 0.27568100 \\
\hline-4.38269800 & -0.70762900 & 3.26044800 \\
\hline-0.70972400 & -1.34525400 & 1.98648900 \\
\hline-0.59741400 & 0.37510800 & 2.71999400 \\
\hline-6.15784100 & 0.17039100 & 1.64142400 \\
\hline-3.49813800 & -2.63955000 & 0.96332700 \\
\hline-5.28610800 & -2.80198600 & 0.74906600 \\
\hline-4.15506900 & -3.14479900 & -0.60341000 \\
\hline-5.38244000 & 3.60638500 & -1.40123000 \\
\hline-4.78118000 & 4.13298200 & 0.20806500 \\
\hline-3.65105600 & 3.40367900 & -0.96926500 \\
\hline-6.45918600 & -0.85808000 & 1.87779600 \\
\hline-6.33666200 & 0.78654300 & 2.53471200 \\
\hline-6.7853610 & 0.53784600 & 0.8251000 \\
\hline
\end{tabular}

\subsection{AU-CATALYST Cycle-O F-MLn}

ENERGY $=-1327.15825$

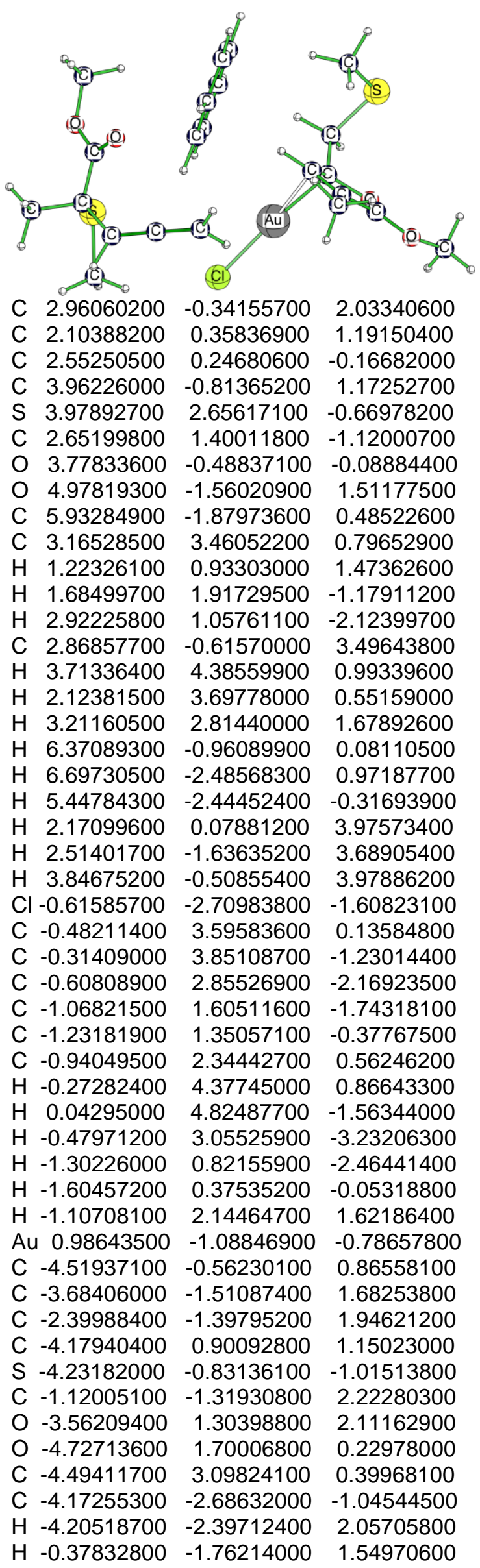




$\begin{array}{rrrr}\text { H } & -0.76329200 & -0.80531600 & 3.11649000 \\ \text { C } & -6.00206400 & -0.77246000 & 1.18067000 \\ \text { H } & -3.29203900 & -3.03848000 & -0.50182500 \\ \text { H } & -5.09646000 & -3.11404500 & -0.64075300 \\ \text { H } & -4.07538200 & -2.96045800 & -2.09946900 \\ \text { H } & -5.05079000 & 3.59425200 & -0.39781000 \\ \text { H } & -4.84762100 & 3.43253600 & 1.38119700 \\ \text { H } & -3.42276100 & 3.31628000 & 0.30579700 \\ \text { H } & -6.29106900 & -1.80647600 & 0.95239500 \\ \text { H } & -6.19220900 & -0.59567800 & 2.24922000 \\ \text { H } & -6.62912100 & -0.10010200 & 0.58735100\end{array}$

\subsection{AU-REGENERATION TSX}

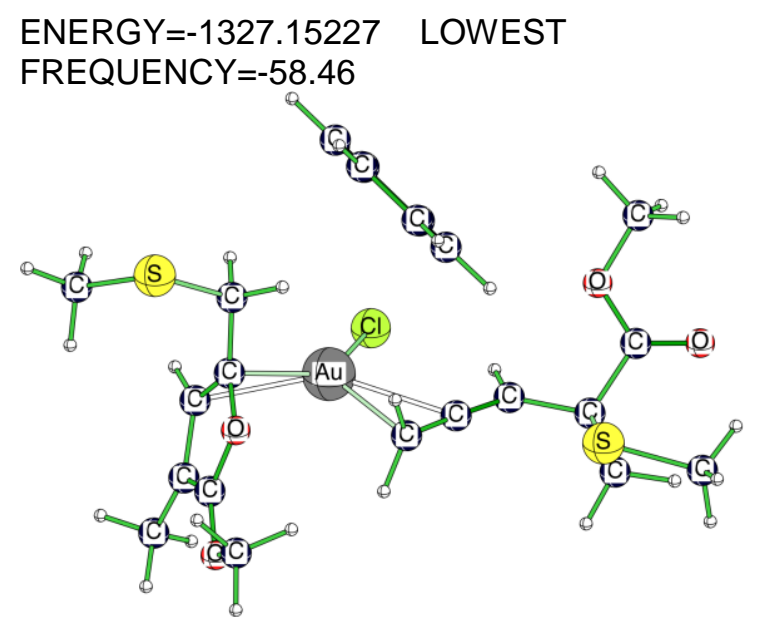

C $3.95451000-1.08964600 \quad 0.03150700$

C $2.55265800 \quad-0.83598700-0.46099100$

$\begin{array}{llll}\text { C } & 1.45739100 & -0.98722300 & 0.24603900\end{array}$

$\begin{array}{llll}\text { C } & 4.85479500 & 0.11630500 & -0.22035900\end{array}$

S $3.89548000 \quad-1.26914800 \quad 1.92827500$

C $0.41000100 \quad-1.14978300 \quad 1.06416100$

$\begin{array}{llll}\text { O } & 6.05930400 & 0.04368900 & -0.35153000\end{array}$

$\begin{array}{llll}\text { O } & 4.16644100 & 1.25895800 & -0.23418400\end{array}$

$\begin{array}{llll}\text { C } 4.94751400 & 2.44815700 & -0.36990900\end{array}$

C $5.65021700 \quad-1.77378800 \quad 2.29467100$

H $2.45612900-0.52657400-1.51050100$

H $-0.04177100-2.13381200 \quad 1.20539900$

H $0.09933100-0.34293000 \quad 1.73272400$

C $4.51472900-2.33475800-0.63941100$

H $5.83917600 \quad-2.79528500 \quad 1.95434400$

H $6.35267700-1.07617000 \quad 1.82886100$

H $5.74687700-1.72523000 \quad 3.38233100$

H $5.52266400 \quad 2.42507200 \quad-1.30126900$

H $4.23602400 \quad 3.27664200-0.38091200$

H $5.63617700 \quad 2.54831200 \quad 0.47599300$

H $3.92358900-3.21004100-0.34695100$

H $4.45478700-2.22491400-1.73048800$

H $5.56559500-2.49389600-0.37877000$

Cl $0.45450700 \quad-0.00172800 \quad-3.19281100$

H $-3.17837700-3.71838800 \quad 3.61978300$

C $-3.07580600 \quad-2.77021100 \quad 3.08936100$

$\begin{array}{llll}\text { O } & -3.23722600 & -3.07832900 & 1.70452100\end{array}$

H $-3.84577900-2.06556400 \quad 3.42541600$

H $-2.08410900-2.34106000 \quad 3.27909500$

C $-3.18891200 \quad-2.06693800 \quad 0.85161400$
C -3.44757200
$-2.07437000$
$-0.49759400$
O -2.77245000
$-0.86717400$
1.30181700
C -3.15863600
$-0.73771600$
$-0.91656900$
C -3.88032200
$-3.23978000$
$-1.32122500$
C -2.70129100
$-0.02731100$
0.20461900
H -3.36790700
$-0.29624800$
$-1.88695200$
H -3.08319200
$-3.56056900$
$-2.00418900$
H -4.13707100
$-4.09001500$
$-0.68043500$
H -4.75859000
$-2.99619300$
$-1.93058000$
C -2.68320100
1.42869000
0.48290300
S -4.23647900
2.06555000
1.33408500
H -2.56093900
1.97225900
$-0.46198600$
H -1.86166300
1.71669800
1.15139200
C -5.46277000
1.64379200
0.00094200
H -6.41667800
H -5.13638400
2.08956500
0.29334900
$2.07117500-0.95339900$
H -5.57901600
0.55859100
$-0.08863900$
Au -0.67959300
$-0.36732800$
C -0.04249000
3.49744200
$-0.98466800$
C -0.72292300
C -0.45910400
4.22472700
$-0.66736200$
3.98844600
0.31466900
C 0.49077700
3.02942900
1.66784700
C 1.17590200
C 0.90611400
2.30673600
2.03835100
2.53826200
1.05625300
H -0.25048000
3.66956800
$-0.29756800$
H -1.46574100
H -0.99302900
4.96844500
$-1.72316600$
4.55113100
0.02861600
H 0.70103700
2.84955400
2.43273200
1.56681500
3.09218800
H 1.92941800
H 1.43642900
1.97485600
1.33492300

\subsection{AU-REGENERATION Furane}

ENERGY $=-1327.16098$

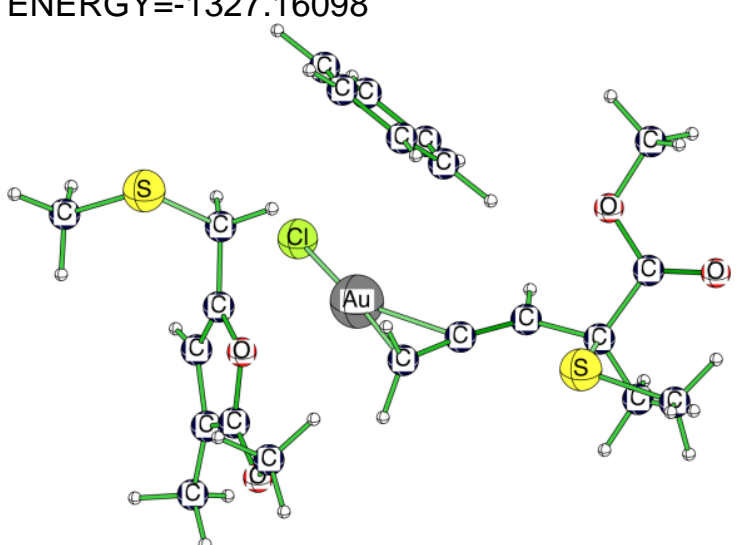

C 3.95955300

$-0.89350800$

$-0.21405100$

C $2.64012600 \quad-0.54192600-0.85675500$

$\begin{array}{llll}\text { C } & 1.45350200 & -0.85569400 & -0.37865900\end{array}$

$\begin{array}{llll}\text { C } & 4.84724800 & 0.34134900 & -0.08324700\end{array}$

S $3.62669600 \quad-1.44639500 \quad 1.57847600$

C $0.42309200 \quad-1.27552400 \quad 0.41088000$

$\begin{array}{llll}\text { O } & 6.05739500 & 0.29559800 & -0.01525500\end{array}$

$\begin{array}{llll}\text { O } & 4.13247800 & 1.46660300 & -0.01220000\end{array}$

C $4.88593100 \quad 2.66177600 \quad 0.21701800$

C $5.31664800 \quad-2.03359300 \quad 2.09363300$

H $2.71093700 \quad-0.00581900-1.80607200$

H $0.05840900 \quad-2.30436900 \quad 0.35977500$

H $0.11807700 \quad-0.67439300 \quad 1.27163100$ 


\begin{tabular}{|c|c|c|}
\hline 4.65315400 & -1.97770300 & -1.02601800 \\
\hline 5.57449000 & -2.96558400 & 1.58446700 \\
\hline 6.06584300 & -1.26201400 & 1.89356000 \\
\hline 5.24545500 & -2.21051100 & 3.16984900 \\
\hline 5.62943600 & 2.80354300 & -0.57381900 \\
\hline 4.16266000 & 3.47990700 & 0.21411200 \\
\hline 5.39474300 & 2.60697900 & 1.18517800 \\
\hline 4.05176200 & -2.89360000 & -1.01081700 \\
\hline 4.76058200 & -1.64899700 & -2.06864400 \\
\hline 5.65572900 & -2.18673800 & -0.64057900 \\
\hline-1.47913600 & 0.90918900 & -3.21906900 \\
\hline-1.72718900 & -4.00451300 & 3.68809300 \\
\hline-2.00039900 & -3.07005900 & 3.19340200 \\
\hline-2.19733000 & -3.39207800 & 1.82116600 \\
\hline-2.91854500 & -2.66643100 & 3.63892000 \\
\hline-1.19100000 & -2.33788700 & 3.31580300 \\
\hline-2.62321900 & -2.40311400 & 1.03042400 \\
\hline-3.12402600 & -2.46647800 & -0.24091000 \\
\hline-2.48831400 & -1.12997300 & 1.46754700 \\
\hline-3.30534000 & -1.09062700 & -0.61654200 \\
\hline-3.37177500 & -3.69677800 & -1.04770100 \\
\hline-2.90361500 & -0.31550800 & 0.43899000 \\
\hline-3.67951500 & -0.71047400 & -1.56230000 \\
\hline-2.74126000 & -3.71827000 & -1.94674300 \\
\hline-3.15059800 & -4.59462200 & -0.45993300 \\
\hline-4.41531200 & -3.75876900 & -1.38017900 \\
\hline-2.87145400 & 1.13590800 & 0.67168800 \\
\hline-4.24784500 & 1.77489500 & 1.79710400 \\
\hline-2.94497000 & 1.66377900 & -0.28765300 \\
\hline-1.94746500 & 1.45513200 & 1.17320500 \\
\hline-5.68172600 & 1.35474600 & 0.69005300 \\
\hline-6.58762700 & 1.71535700 & 1.18375700 \\
\hline-5.56421100 & 1.85417600 & -0.27755000 \\
\hline H -5.74457300 & 0.27096400 & 0.54860400 \\
\hline $\mathrm{Au}-0.41438600$ & -0.21824500 & -1.35052600 \\
\hline C -0.24822800 & 3.53296000 & -0.24359800 \\
\hline-0.99875200 & 3.99821800 & 0.84031400 \\
\hline-0.67728800 & 3.59447800 & 2.14024200 \\
\hline 0.39750800 & 2.72553300 & 2.35766900 \\
\hline 1.15354100 & 2.26410500 & 1.27552500 \\
\hline 0.82842600 & 2.66715800 & -0.02465200 \\
\hline-0.50475900 & 3.83398600 & -1.25833500 \\
\hline-1.84518900 & 4.66303900 & 0.67259900 \\
\hline-1.27011800 & 3.95065500 & 2.98191100 \\
\hline 0.64799000 & 2.41150000 & 3.37027800 \\
\hline 2.00077500 & 1.59499600 & 1.43912300 \\
\hline 1.42435200 & 2.31260000 & -0.86808600 \\
\hline
\end{tabular}

\section{Pt CATALYST}

\subsection{PT-CATALYST TS1}

ENERGY $=-1325.76793 \quad$ LOWEST FREQUENCY $=-260.70$

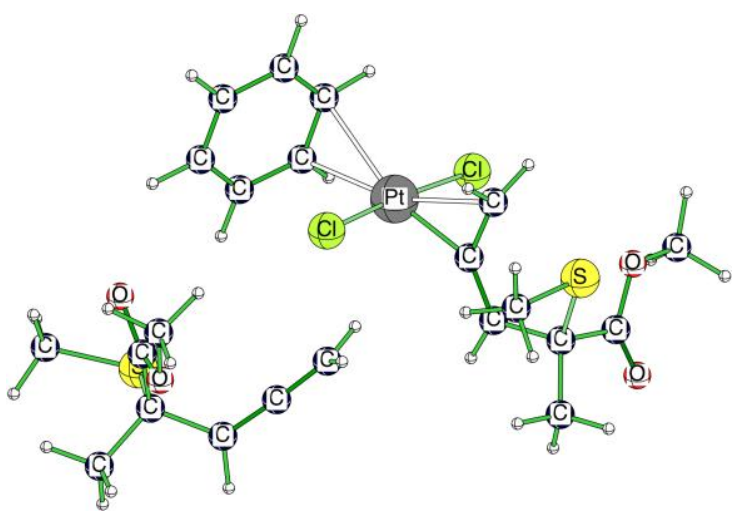

C $-4.76879800-2.04459300 \quad-0.31996500$

C $-3.52148000-2.81124500-0.69389100$

C $-2.31805700-2.28324100-0.63898400$

C $-4.47677700 \quad-0.98061400 \quad 0.74473000$

S $-5.30873800-1.23840300-1.94501500$

C $-1.14919000 \quad-1.70587200-0.49879700$

$\begin{array}{llll}\text { O } & -4.74267300 & 0.19959800 & 0.66509600\end{array}$

O $-3.92204100 \quad-1.54238900 \quad 1.82073000$

C $-3.52293900 \quad-0.63521000 \quad 2.85316600$

C $-6.97472300-0.53376600-1.50399000$

H $-3.67270600-3.82713600-1.06713500$

H $-0.69422300-1.12762000-1.30813400$

H $-0.60780300-1.76588500 \quad 0.44796800$

$\begin{array}{llll}\text { C }-5.83727300 & -2.99676300 & 0.21599600\end{array}$

H $-6.87552600 \quad 0.12424400 \quad-0.63744600$

H $-7.29050600 \quad 0.04961700 \quad-2.37315600$

H $-7.70365200-1.32819500-1.31936600$

H $-4.36709900-0.01233400 \quad 3.16871000$

H $-3.17683700 \quad-1.25733900 \quad 3.68096600$

H $-2.70323200 \quad 0.00332700 \quad 2.49571500$

H $-6.09673100-3.73383100-0.55367500$

H $-5.45563300-3.52362400 \quad 1.09916200$

H $-6.74895400-2.45967700 \quad 0.50562900$

Cl $1.88361200 \quad 1.48068800 \quad-2.30991600$

$\mathrm{Cl}-0.04328000 \quad 0.79608000 \quad 2.10264100$

C $-2.30572100 \quad 1.87504800-0.40353800$

C $-2.67910400 \quad 2.57065300 \quad 0.73700600$

C $-1.91875900 \quad 3.66724700 \quad 1.18104800$

C $-0.78114500 \quad 4.06453200 \quad 0.48945500$

C $-0.38843100 \quad 3.36914500-0.66977500$

C $-1.15005600 \quad 2.26322800 \quad-1.11864900$

H $-2.91447800 \quad 1.04113400 \quad-0.75386300$

H $-3.57209200 \quad 2.25996800 \quad 1.27764300$

H $-2.22403500 \quad 4.20846900 \quad 2.07531700$

H $-0.19958100 \quad 4.92052200 \quad 0.82654500$

H $\quad 0.43512400 \quad 3.73679400 \quad-1.27976900$

H $-0.94229400 \quad 1.82641500-2.09618400$

Pt $0.82088800 \quad 1.19818600 \quad-0.13715200$

$\begin{array}{llll}\text { C } 3.78682200 & -2.05701800 & 0.33471000\end{array}$

C $2.42775600-1.45627300 \quad 0.00865900$

$\begin{array}{lll}\text { C } 2.21479200 & -0.17145600 & 0.25851900\end{array}$

$\begin{array}{llll}\text { C } 4.56750400 & -2.01909500 & -0.98419500\end{array}$

S $4.70010300 \quad-0.93013800 \quad 1.56782700$

C $3.03721100 \quad 0.81242200 \quad 0.85682400$

$\begin{array}{llll}\text { O } & 4.64598500 & -2.96511600 & -1.73433900\end{array}$

$\begin{array}{llll}\text { O } & 5.05773000 & -0.80605100 & -1.23307300\end{array}$

C $5.64140200-0.62632900-2.52801700$

$\begin{array}{llll}\text { C } & 3.74148100 & -1.29969100 & 3.11873900\end{array}$ 


$\begin{array}{rrrr}\mathrm{H} & 1.71818700 & -2.10270500 & -0.51021300 \\ \mathrm{H} & 2.84566300 & 1.15337200 & 1.87492100 \\ \mathrm{H} & 3.68817300 & 1.42349400 & 0.22998700 \\ \mathrm{C} & 3.66503400 & -3.48053900 & 0.84783600 \\ \mathrm{H} & 2.66749600 & -1.22320800 & 2.91378400 \\ \mathrm{H} & 4.00333800 & -2.29069600 & 3.49532800 \\ \mathrm{H} & 4.03757900 & -0.53725300 & 3.84335000 \\ \mathrm{H} & 4.88242300 & -0.77643300 & -3.30267400 \\ \mathrm{H} & 6.00721500 & 0.40141100 & -2.55129300 \\ \mathrm{H} & 6.46380600 & -1.33281300 & -2.67895000 \\ \mathrm{H} & 3.01903000 & -3.52277000 & 1.73167600 \\ \mathrm{H} & 3.20676100 & -4.09615600 & 0.06517300 \\ \mathrm{H} & 4.64450100 & -3.90981000 & 1.08791700\end{array}$

\subsection{PT-CATALYST Allene MLn-A}

ENERGY $=-1325.77428$

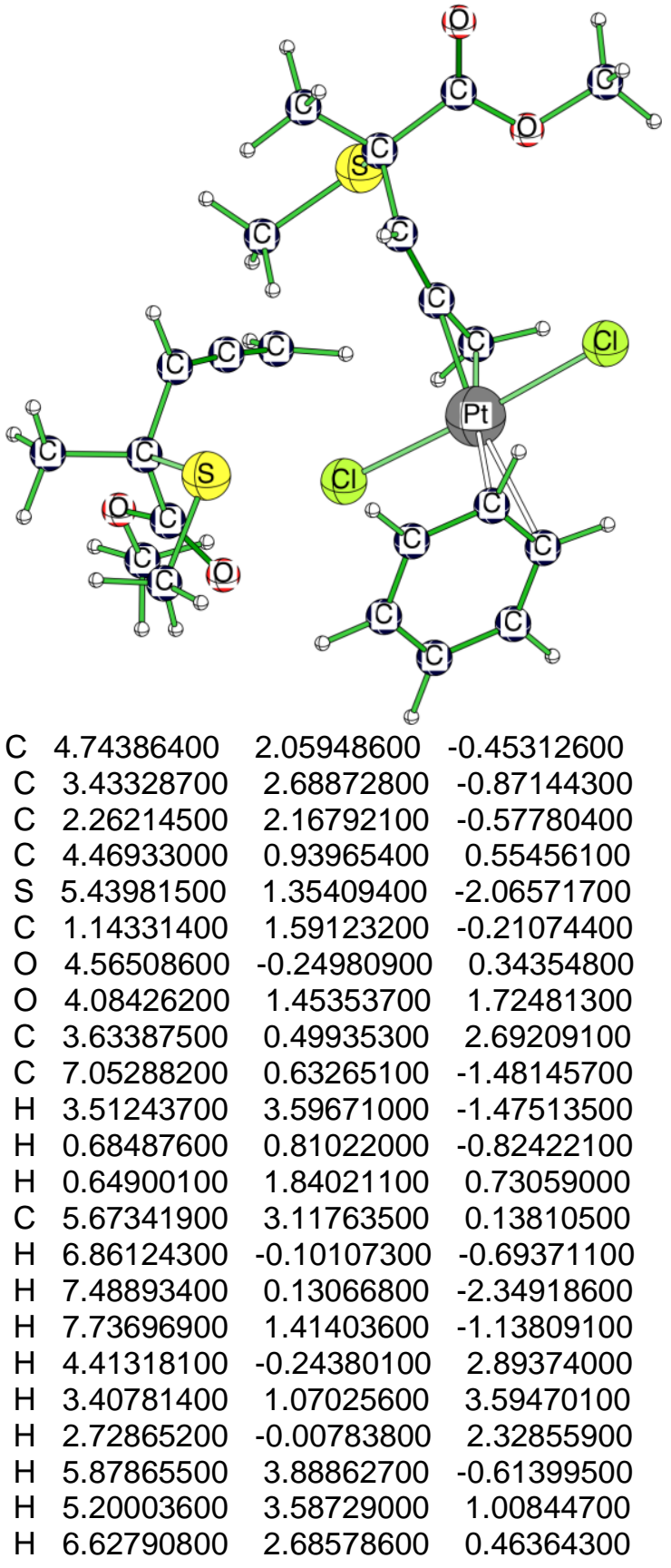

Cl $-2.33084500 \quad-1.95510200 \quad-2.01646200$

Cl $0.07979800 \quad-0.94416700 \quad 2.06710000$

C $2.06851700-1.96911700-0.59169400$

C $2.60098200 \quad-2.60237100 \quad 0.52417000$

C $1.95081100 \quad-3.71228100 \quad 1.08817200$

C $0.76873400 \quad-4.19761900 \quad 0.53897000$

C $0.22501100-3.57832000-0.60050800$

C $0.87303400-2.45056400-1.16610200$

H $2.58904500 \quad-1.11563400-1.02846900$

H $3.52768900 \quad-2.22138400 \quad 0.94941900$

H $2.37667900 \quad-4.19623900 \quad 1.96558300$

H $\quad 0.27125500 \quad-5.06275800 \quad 0.97239000$

H $-0.63277000-4.01598100-1.10945900$

H $0.52588200 \quad-2.05913500-2.12308500$

Pt $-1.07386400 \quad-1.49830500 \quad 0.00719800$

C $-3.28546000 \quad 2.41619000 \quad 0.01562600$

C $-2.24564300 \quad 1.34444600 \quad-0.23217000$

C $-2.30773300 \quad 0.13525000 \quad 0.28651500$

C $-4.40381800 \quad 2.25755700 \quad-1.02324500$

S $-4.13952400 \quad 2.20998600 \quad 1.70429200$

C $-2.88957800 \quad-0.89759400 \quad 1.00729100$

O $-4.71623100 \quad 3.11370500 \quad-1.82012000$

O $-4.96459800 \quad 1.05043400 \quad-0.95374600$

C $-5.97398500 \quad 0.78362600 \quad-1.92931500$

C $-2.67509900 \quad 2.47734600 \quad 2.82005100$

H $-1.43250500 \quad 1.61183800 \quad-0.91094700$

H $-2.72607500 \quad-0.98372100 \quad 2.08270400$

H $-3.72381800 \quad-1.44538000 \quad 0.56499200$

C $-2.64538400 \quad 3.78903300 \quad-0.10987800$

H $-1.84223500 \quad 1.83627800 \quad 2.50470000$

H $-2.37419600 \quad 3.52811500 \quad 2.83473200$

H $-3.00177000 \quad 2.17572900 \quad 3.81863000$

H $-5.55291200 \quad 0.85286500 \quad-2.93784600$

H $-6.31983200-0.23278100-1.73272000$

H $-6.79920000 \quad 1.49708300 \quad-1.83239500$

H $-1.81225700 \quad 3.88935100 \quad 0.59730300$

H $-2.24520300 \quad 3.92230100-1.12316300$

H $-3.37612000 \quad 4.58205400 \quad 0.07842800$

\subsection{PT-CATALYST S-Cycle}

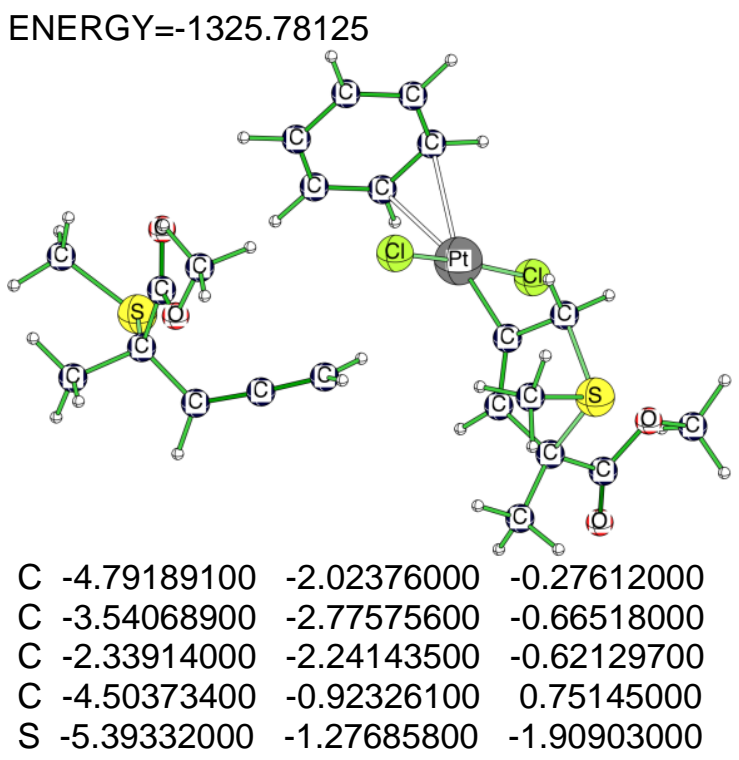




\begin{tabular}{|c|c|c|}
\hline & -1.65631800 & -0.49177300 \\
\hline O -4.83758300 & 0.23858400 & 0.66020100 \\
\hline O -3.87296000 & -1.42973700 & \\
\hline-3.46516400 & -0.47468300 & 2.79850900 \\
\hline C -7.06698000 & -0.59925000 & -1.45486500 \\
\hline H -3.69060800 & -3.78994700 & -1.04422400 \\
\hline H -0.73444300 & -1.06094800 & -1.29732200 \\
\hline H -0.62067000 & -1.71130500 & 0.44915300 \\
\hline C -5.82366100 & -2.98449400 & 0.31493800 \\
\hline H -6.96457800 & 0.09924900 & -0.62126800 \\
\hline H -7.41957800 & -0.06415900 & -2.34091300 \\
\hline H -7.76934000 & -1.40357600 & -1.21771700 \\
\hline H -4.32124300 & 0.11800300 & 3.13938900 \\
\hline H -3.04785000 & -1.05546000 & 3.62362600 \\
\hline H -2.69524100 & 0.18932500 & 2.38196600 \\
\hline H -6.08566200 & -3.75013600 & -0.42561500 \\
\hline H -5.40537900 & -3.47697500 & 1.20155600 \\
\hline H -6.73986800 & -2.46077000 & 0.61454300 \\
\hline Cl 1.89610200 & 1.40713100 & -2.34694300 \\
\hline Cl 0.06708300 & 0.81528600 & 2.14183600 \\
\hline C -2.38286400 & 1.88404300 & -0.46002400 \\
\hline C -2.74530300 & 2.60389400 & 0.66934600 \\
\hline C -1.94808300 & 3.67440100 & 1.11568500 \\
\hline C -0.79258100 & 4.02126000 & 0.43057100 \\
\hline C -0.41497300 & 3.30220700 & -0.72324900 \\
\hline C -1.21379400 & 2.22983400 & -1.17136500 \\
\hline H -3.01929400 & 1.07042200 & -0.80928700 \\
\hline H -3.65449400 & 2.32932000 & 1.20277600 \\
\hline$H-2.24020000$ & 4.23255800 & 2.00417600 \\
\hline H -0.18061200 & 4.85656800 & 0.76707500 \\
\hline H $\quad 0.41935500$ & 3.64488800 & -1.33343700 \\
\hline H -0.99888100 & 1.75859000 & -2.13029100 \\
\hline Pt 0.84529100 & 1.17278300 & -0.15224000 \\
\hline C 3.73277000 & -2.06385300 & 0.29247400 \\
\hline C 2.42424700 & -1.37901500 & -0.01953000 \\
\hline C 2.28941600 & -0.08150000 & 0.28743400 \\
\hline C 4.57259900 & -2.01751100 & -0.98729500 \\
\hline S 4.55450300 & -0.86637900 & 1.56626400 \\
\hline C 3.41071600 & 0.57621800 & 1.01177100 \\
\hline O 4.61606500 & -2.93895000 & -1.76759300 \\
\hline O 5.13868900 & -0.82630800 & -1.16332900 \\
\hline C 5.74505300 & -0.60933600 & -2.44634800 \\
\hline C 3.63664800 & -1.31261900 & 3.11914900 \\
\hline H 1.69306400 & -1.95887600 & -0.58390600 \\
\hline H 3.10838600 & 1.09602700 & 1.92808600 \\
\hline H 4.01830400 & 1.22267900 & 0.36892400 \\
\hline C 3.60822900 & -3.47093500 & 0.83452900 \\
\hline 2.56658000 & -1.34837400 & 2.89118800 \\
\hline H 4.01762800 & -2.26883000 & 3.48103700 \\
\hline H 3.85468300 & -0.51840500 & 3.83634300 \\
\hline H 4.99490700 & -0.73259300 & -3.23324200 \\
\hline H 6.11573000 & 0.41633800 & -2.43183900 \\
\hline H 6.56507100 & -1.31714900 & -2.60219800 \\
\hline H 2.90960700 & -3.51031000 & 1.67681500 \\
\hline H 3.20298200 & -4.09637800 & 0.03122200 \\
\hline H 4.57636000 & -3.89365400 & 1.12911700 \\
\hline
\end{tabular}

\section{$2.4 \quad$ PT-CATALYST TS2}

ENERGY $=-1325.76007 \quad$ LOWEST

FREQUENCY $=-241.16$

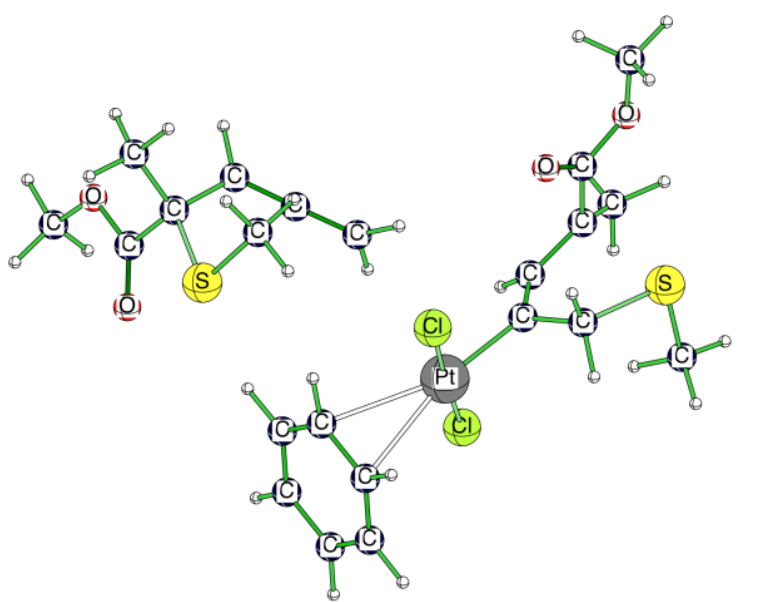

C -4.41136700

$1.93175000 \quad 0.31621100$

C $-3.25421400 \quad 2.56018900 \quad-0.44489200$

C $-2.08318700 \quad 2.00106200 \quad-0.65877300$

C $-5.27629300 \quad 1.25528400 \quad-0.74830700$

S -3.87183200 $0.54031000 \quad 1.47390200$

C $-0.90083000 \quad 1.46111700 \quad-0.82840400$

$\begin{array}{llll}\text { O } & -5.31267000 & 0.06911600 & -0.98590000\end{array}$

$\begin{array}{llll}\mathrm{O} & -5.96184600 & 2.17232000 & -1.44532500\end{array}$

C $-6.72814800 \quad 1.65387200-2.53420700$

C $-2.80800000 \quad 1.50545100 \quad 2.65591600$

H $-3.46663100 \quad 3.54575100-0.87170600$

H $-0.09353500 \quad 1.62675100 \quad-0.10985600$

H $-0.69914900 \quad 0.79902600 \quad-1.67313900$

C $-5.18129800 \quad 3.01050600 \quad 1.07098500$

H $-3.41621000 \quad 2.10106100 \quad 3.34271300$

H -2.22858100 $0.76204500 \quad 3.21205000$

H $-2.10835400 \quad 2.13879800 \quad 2.10063500$

H $-7.46848000 \quad 0.93256000-2.17257500$

H $-7.22343000 \quad 2.51277000-2.99165300$

H $-6.07369100 \quad 1.15880200 \quad-3.25961800$

H $-4.52554700 \quad 3.49574000 \quad 1.80379800$

H $-5.54130600 \quad 3.77968200 \quad 0.37685800$

H $-6.04139300 \quad 2.58278800 \quad 1.59874200$

Cl $0.55227200 \quad-0.33182300 \quad 2.53471400$

Cl $1.11508500-2.23919500-1.92769500$

C $-2.33632500 \quad-2.31022900-0.45182000$

C $-2.25240800 \quad-3.48275900-1.18896500$

C $-1.41367500-4.52973300 \quad-0.76269500$

C $-0.66301500 \quad-4.40105600 \quad 0.39791500$

C $-0.74275200 \quad-3.21669300 \quad 1.15929100$

C $-1.58384200 \quad-2.17133200 \quad 0.73238200$

H $-3.00134900-1.49923000-0.75007600$

H $-2.83809800-3.59541100 \quad-2.09996600$

H $-1.35335700-5.44526100 \quad-1.34928500$

H $-0.02129700-5.21503500 \quad 0.73083300$

H $-0.24754400 \quad-3.15206900 \quad 2.12758800$

H $-1.75113500-1.30058200 \quad 1.36822000$

Pt $0.78630100-1.30763200 \quad 0.29164000$

C $3.42300400 \quad 1.69366100-1.21341000$

C $2.42192300 \quad 0.68909000 \quad-1.12785700$

$\begin{array}{llll}\text { C } & 2.28266300 & -0.12908800 & -0.03409700\end{array}$

C $3.42010900 \quad 2.77395900 \quad-0.14578800$

$\begin{array}{llll}\text { S } & 5.01154100 & 0.49084600 & 0.18358700\end{array}$ 

C 3.43440400
$-0.22562700$
0.93675900
O 2.72595200
2.74273700
0.84554400
O 4.26661000
3.75682400
$-0.45214800$
C 4.33814300
4.81825800
0.50715000
C 5.33423500
$-0.89012900$
$-1.01194500$
H 1.85584800
0.46045200
$-2.03412400$
H 3.64744000
H 3.22447100
$-1.26982600$
1.18914000
0.33081300
1.85838100
C 3.99400600
2.04509200
$-2.54706500$
H 4.41500600
$-1.07004100$
$-1.58670100$
H 6.15902800
$-0.58881500$
$-1.66046100$
H 5.59866400
$-1.78446700$
$-0.44213000$
H 4.69946400
4.43577400
1.46761600
H 5.03998000
H 3.35127100
5.54626400
0.09709500
H 4.01677200
5.27015200
0.64896500
H 3.36059800
$1.17175700-3.20717900$
H 4.99551500
2.81346000
$-3.01364100$
$2.47652100-2.45527500$

\subsection{PT-CATALYST Cycle-O F-MLn}

ENERGY $=-1325.81441$

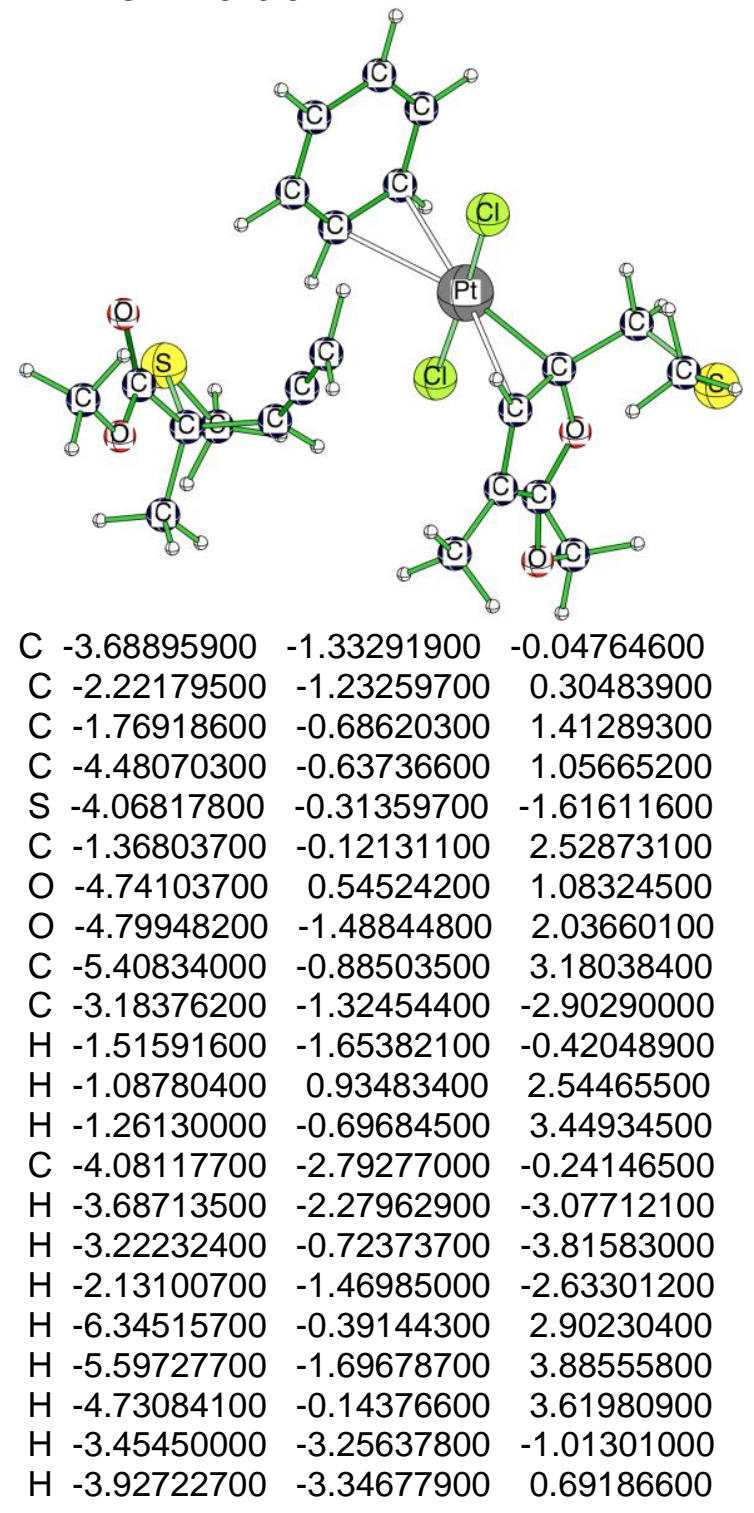

H $-5.13252500-2.88507000-0.54009500$

Cl $0.44493600-0.52496800 \quad-2.41212700$

Cl $1.31838800 \quad 2.19703200 \quad 1.56806500$

C $-1.93184600 \quad 2.56225400 \quad-0.02479700$

C $-1.56648900 \quad 3.81244100 \quad 0.44397500$

C $-0.48831600 \quad 4.51092500 \quad-0.14231700$

C $0.21372600 \quad 3.95946700 \quad-1.19835800$

C $-0.14385300 \quad 2.68035200 \quad-1.69273700$

C $-1.22267800 \quad 1.98308700-1.10137600$

H $-2.78476000 \quad 2.02710100 \quad 0.39607100$

H $-2.11730200 \quad 4.26732600 \quad 1.26590900$

H $-0.21353300 \quad 5.49386300 \quad 0.23679300$

H $1.03231500 \quad 4.50477600 \quad-1.66454800$

H $0.27946100 \quad 2.32231600-2.63117000$

H $-1.61308100 \quad 1.07714200-1.56701100$

Pt $0.94393500 \quad 0.89472100 \quad-0.46563000$

C $1.53515100 \quad-2.35933000 \quad 0.88463000$

C $1.77314600 \quad-1.05019300 \quad 1.23301900$

$\begin{array}{lll}\text { C } 2.50238900 & -0.36914500 & 0.17052600\end{array}$

$\begin{array}{llll}\text { C } & 2.16524100 & -2.49330000 & -0.37704100\end{array}$

S $5.12080400 \quad-0.64944000 \quad 1.22126100$

$\begin{array}{llll}\text { C } 3.79072600 & 0.38254000 & 0.38718500\end{array}$

$\begin{array}{llll}\text { O } & 2.77278100 & -1.41667700 & -0.78700000\end{array}$

$\begin{array}{llll}\text { O } & 2.14617600 & -3.55902100 & -1.11460200\end{array}$

C $2.73025300-3.45849300-2.43079300$

$\begin{array}{llll}\text { C } 4.57849500 & -0.42962200 & 2.98964500\end{array}$

H $1.45905300 \quad-0.54277000 \quad 2.14091200$

H $3.62264800 \quad 1.27761800 \quad 0.99159900$

H $\quad 4.20363700 \quad 0.67926700 \quad-0.58271900$

C $0.76563900 \quad-3.43489000 \quad 1.57139900$

H $3.72451500 \quad-1.07266500 \quad 3.22436400$

H $5.42711700-0.70596200 \quad 3.62051200$

H $\quad 4.32039700 \quad 0.62028400 \quad 3.16439200$

H $3.80454400-3.26873600-2.34692600$

H $2.54626600 \quad-4.42237500 \quad-2.90552800$

H $2.23931900-2.64475000-2.97758500$

H $0.36920000 \quad-3.07311600 \quad 2.52484200$

H $-0.07742600-3.77685800 \quad 0.95750100$

H $1.39996600-4.30679000 \quad 1.77189100$

\subsection{PT-REGENERATION TS $x^{\prime}$}

\section{ENERGY $=-1325.79899 \quad$ LOWEST}

FREQUENCY $=-40.52$

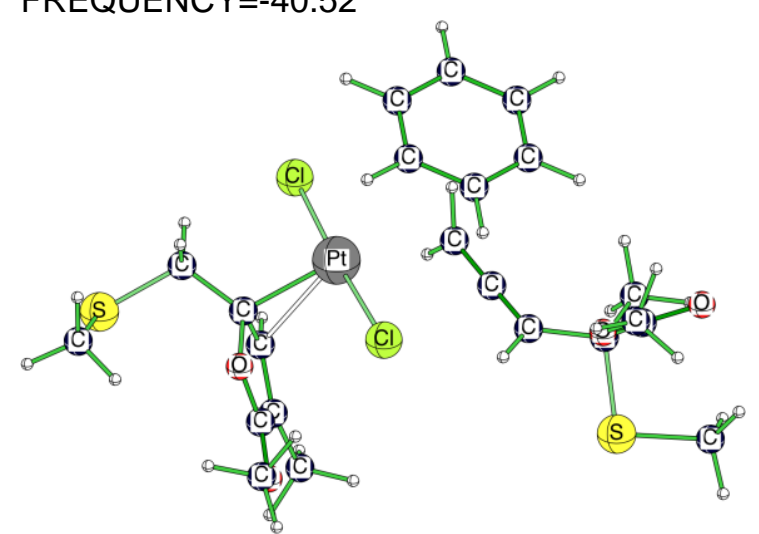

C $4.14953000-0.77362100 \quad-0.68474700$

C $2.66732600-0.59997400-0.51787300$

$\begin{array}{llll}\text { C } & 1.94831500 & 0.23804500 & -1.22759600\end{array}$ 

C $4.88936100-0.48360600$
S $4.38799100-2.65603600$
0.61787700
C 1.220233001 .11142700

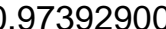
O 6.05209600
$-0.13475900$
$-1.89220900$
0.67168000
1.69584400
O 4.12380500
$-0.67453900$
C $4.76210200-0.45422500$
C $6.21873800-2.75559900$
H 2.17048200
$-1.15677100$
2.95214300
$-1.30910800$
0.28181000
H 1.17041800
2.15242700
H 0.69143800
0.84708000
$-1.55995300$
C 4.73464400
0.03747600
$-2.80748800$
H 6.45448200
$-2.35091500$
$-1.82388700$
$-2.29679400$
H 6.77792800
$-2.22530300$
$-0.53224700$
H 6.46935700
$-3.81934300$
H 5.16180000
0.56487800
H 3.98996600
$-0.60130200$
.
3.00745800
3.71022900
H 5.58041700
$-1.16763400$
3.09879800
H 4.54066900
1.10669100
$-1.65589500$
H 4.26763500
$-0.25589300$
$-2.77105900$
H 5.81829300
$-0.09772700$
$-1.89298900$
$\mathrm{Cl}-0.15880500$
$-0.82523300$
Cl -1.89813900
2.34081700
H -2.25278000
$-4.46895000$
1.53868700
$-1.75658000$
2.59047800
2.23853700
O -2.40876100
$-3.48126000$
$-3.52584600$
0.80350900
H -3.59282200
$-3.27788200$
H -1.88978900
$-2.70331000$
2.50429800
2.63799300
0.14713700
C -2.31994100
$-2.42306300$
$-1.23534700$
O -2.97916300
$-2.25350100$
0.75936100
C -2.57222700
C -1.79843700
$-0.91916800$
$-1.45581900$
C -2.90546800
$-3.31435000$
$-2.14084400$
H -2.54779900
$-0.25807500$
$-0.19326600$
H -0.76186800
$-0.38824600$
$-2.40308100$
H -2.39358000
$-3.56658000$
$-1.88276200$
H -1.81748500
$-4.23132900$
$-2.06274700$
C -4.10523900
$-2.97659500$
$-3.18083600$
S -5.71753200
$-0.27510000$
$-0.01864000$
H -4.05504100
1.45754400
$-0.35237700$
H -4.14024800
1.03698200
$-0.72997300$
0.99856500
C -6.13041600
$-0.84804200$
1.36996400
H -7.12786100
H -5.41038500
H -6.14780800
$-1.29194200$
1.31976300
1.71836900
0.00836000
2.05070600
Pt -1.08731000
0.73054000
$-0.10726600$
C 0.09886700
3.84122100
0.72537800
C 0.32141600
2.71233400
1.52711100
C 1.60571600
2.15392600
1.60788100
C 2.66125200
C 2.43660100
C 1.15451900
.
0.89456800
3.85066300
4.40612100
0.09525800
H -0.90002800
4.26794300
0.00670200
H -0.48667800
H 1.77236800
H 3.66147200
(
0.65529400
1.26871500
2.21950200
$2.29621600 \quad 0.96559100$
H 3.26060400
4.29311300
$-0.46365700$
H 0.97692400
5.27528900

2.7

PT-REgENERATION Complexe FMLn-A

ENERGY $=-1325.83222$

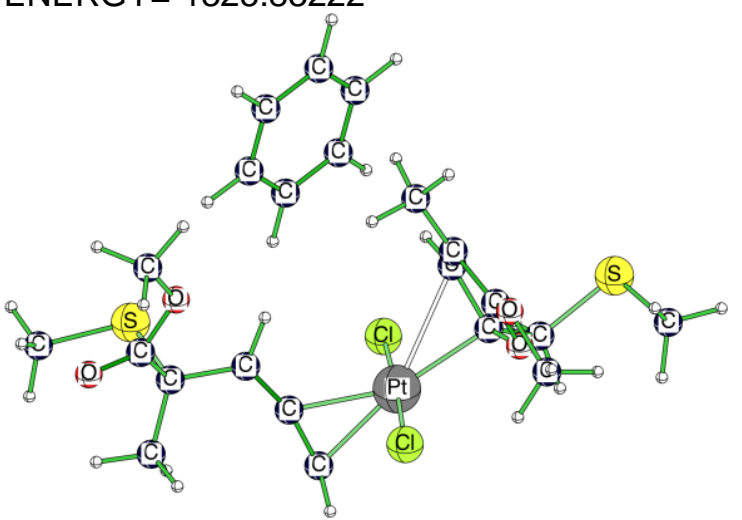

Cl 0.92588500

$-2.31967000$

1.58147600

$\mathrm{Cl} 0.50057400$

$-0.21216300$

$-2.79080900$

C -1.41902500

$2.58909600-1.25203700$

C -0.38386800

C -0.05647700

$3.37623300-1.76728400$

C -0.76704600

$4.59103000-1.15672900$

C -1.80300000

5.02112900

$-0.03053300$

C -2.12967700

$\begin{array}{ll}4.23486100 & 0.48333700\end{array}$

H -1.66708900

$3.01944400-0.12719200$

H 0.16324000

$1.64342000-1.73848700$

H 0.74839400

$\mathrm{H}-0.51617700$

3.03625300

$-2.64687900$

H -2.94689800

5.20407900

$-1.56034300$

H -2.36034000

5.97001700

0.44235100

$2.41010400 \quad 0.26147400$

C -3.67394100

4.57167100

1.35724600

C -2.19758500

$-1.05886300$

0.09678600

C -1.34869000

$-0.79547800$

$-0.00727300$

C -4.25676400

$-1.64817800$

$-0.54609700$

S -4.45205600

$-0.52525900$

1.40018000

C -0.76591600

0.06274300

$-1.25981900$

O -5.26998300

$-2.73733400$

$-1.13812000$

O -3.56083400

$-0.95601100$

1.91041900

C -4.11614200

0.50603200

1.89079300

C -6.25640900

1.12813600

3.04892300

H -1.82205800

$-0.39314600$

$-1.14013200$

H -0.54643200

0.15676600

0.37488300

H -0.76328700

$-3.62727200$

$-0.54680500$

C -4.04932800

$-2.82395700$

$-2.22539500$

H -6.43375400

$-2.51144000$

$-0.11906400$

H -6.59041800

$-1.38185400$

$-1.57091200$

H -6.78935300

$-0.35947000$

$-0.09860500$

H -4.20120700

0.36336500

$-1.72214700$

H -3.42661100

0.40740200

3.86866500

H -5.10805200

1.93248500

3.31635500

H -3.75102700

1.53621000

2.82559000

H -3.53498700

$-2.83226400$

$-1.12372600$

H -5.12564900

$-3.14074300$

0.61958500

Pt 0.76528400

$-2.66380200$

0.00124900

H 3.79063900

$-1.23937900$

$-0.59960600$

C 3.70210600

$-0.86199500$

4.60338800

O 2.97528600

$-0.90552700$

3.51776800

H 4.69345500

0.27972300

3.14187000

H 3.13472500

C 2.69628500

$-0.90461000$

3.05207400

3.20252700

0.43635900

1.88351700 

C 1.86968200
1.44829800
1.34420000
O 3.17099400
$-0.39331500$
0.99027400
C 1.84479400
1.16832900
$-0.00440000$
C 1.13338300
2.48014100
2.12499000
C 2.56363500
$-0.05917600$
$-0.26916000$
H 1.33064800
1.73483700
$-0.77788100$
H $\quad 0.27645000$
2.02896600
2.64428100
H 1.77523000
2.94857300
2.87977100
H 0.74449400
3.25801600
1.45686800
C 3.56616400
$-0.20210000$
$-1.37470200$
S 5.02361500
0.97511100
$-1.22960000$
H 3.08754200
0.03744300
$-2.32955300$
H 3.95730800
$-1.22555600$
$-1.42574900$
C 6.07839000
0.06424400
0.00761400
H 7.11750100
0.33635900
$-0.19398200$
H 5.81529100
0.33997600
1.03270700
H 5.95440600

\subsection{PT-REGENERATION TS $x$ "}

ENERGY $=-1325.80524 \quad$ LOWEST FREQUENCY $=-32.83$

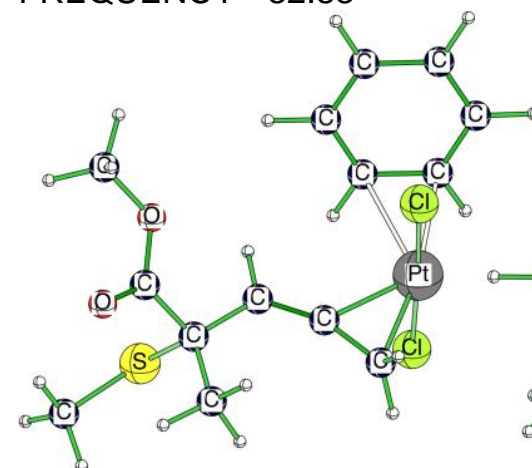

Cl 0.34792400

1.38824900

1.50300600

Cl 0.20894100

$-1.32683800$

$-2.51031100$

C -0.71754100

$1.94297600-2.10300000$

C 0.69304700

1.96794900

$-2.18128900$

C 1.40066800

3.03597300

$-1.59026400$

C 0.71770000

4.04337600

$-0.92455800$

C -0.68499900

C -1.39994900

$4.00475900-0.83869900$

H -1.26813700

2.96401800

$-1.42120800$

$1.17316200-2.64308000$

H 1.20672600

1.26249400

$-2.83488200$

H 2.48598300

$3.07326500-1.67774700$

H 1.26644700

H -2.48789200

4.86518

$-0.46713700$

$2.94635000-1.36671300$

H -1.21432000

4.79831600

$-0.31383500$

C -3.87552700

$-1.08935300$

0.53894600

C -2.67626400

$-0.38714700$

$-0.02958400$

C -1.43210800

$-0.78313700$

0.16006500

C -4.79025500

$-0.12670700$

1.28982700

S $-4.87945000-1.59357900-1.01950900$

C $-0.37613200 \quad-1.57378800 \quad 0.64533900$

$\begin{array}{llll}\text { O } & -5.57602400 & -0.48050800 & 2.14472100\end{array}$

O -4.66166200

1.13580500

0.87451300

C -5.54753000

2.07325100

1.48787700

C -6.30322700

$-2.52921000$

$-0.26208900$

$\mathrm{H}-2.86180700$

0.49933800

$-0.63749000$

1.68146000
H $-0.15266100-2.51711300$

C $-3.53906700-2.29505100$

H $-5.97111600-3.50083300$

H $-6.76028600-1.94150800$

H $-7.02401700-2.67367800$

H $-5.42129200 \quad 2.06554100$

H $-5.28044200 \quad 3.05018500$

H -6.587885001 .83040200$

H $-3.01237900-3.04814600$

H $-2.89939400-1.99328900$

H $-4.44502800-2.74012000$

Pt $0.32300000 \quad 0.05528700$

H 2.91860500

C 2.79875000

O 3.56856700

H 3.16557100

H 1.73970300

C 3.47788300

C 3.75117000

O 3.06962800

C 3.45082800

C 4.15557000

C 3.02422000

H 3.59700100

H 3.27527600

H 4.75945200

H 4.74626900

C 2.96068500

S 4.65700800

H 2.49285800

H 2.39153500

C 5.34670900

H 6.28500800

0.49528200

0.02123700

0.79977000

$-1.01182200$

0.03016400

0.47065100

1.23662400

$-0.77154800$

0.38010000

2.67259700

$-0.83297200$

0.58585500

3.33192000

2.90124100

2.93098900

$-2.19316400$

$-2.98043600$

$-2.18895200$

$-2.86175100$

$-2.84463000$

$-3.40519500$

0.14254200

1.39237600

0.11271000

0.53982900

$-1.07119000$

2.57530600

1.08017600

1.24544900

0.79492000

2.23306700

1.81346100

$-0.51335200$

4.82738100

3.85088400

2.93763200

3.89446000

3.55571900

1.65476500

0.54861500

1.33185500

$-0.55310800$

0.56218300

$-0.04819900$

$-1.61021000$

0.58818400

1.44761800

$-0.32505400$

$-0.61745900$

$-0.90833300$

$-1.60725400$

0.04121900

0.81453900

0.82269100

H $5.54053300-1.79797000 \quad 1.06967900$

H $4.64908900-3.28529700 \quad 1.53427600$

\subsection{PT-REGENERATION Furane}

\section{ENERGY=-1325.81022}

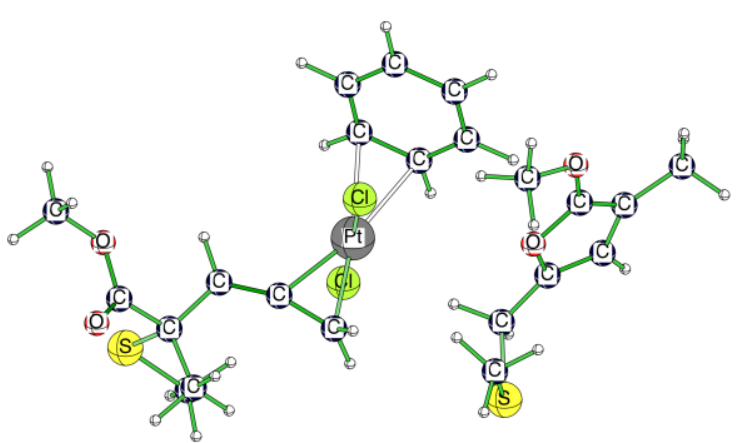

Cl $-0.24359100 \quad-1.31981400 \quad 1.59481900$

Cl $0.66229200 \quad 0.86732500 \quad-2.63672300$

C $-0.41496800 \quad-2.30954200-1.90713500$

C $-1.53482000-1.44014800-1.89541900$

C $-2.65993300-1.75612400-1.10427600$

C $-2.66351300 \quad-2.91313300-0.33846500$

C $-1.54698600-3.76795600 \quad-0.34260200$

C $-0.43013100 \quad-3.47197600-1.11475600$

H $0.40081700-2.12578000-2.60595400$ 


\begin{tabular}{|c|c|c|c|}
\hline & & -0.61978200 & -2.61102400 \\
\hline & -3.53192100 & -1.10140600 & -1.12668100 \\
\hline & -3.53855000 & -3.16325900 & 0.26106700 \\
\hline & 0.42855100 & -4.13980600 & -1.12053100 \\
\hline & -1.55874000 & -4.67101800 & 0.26481900 \\
\hline C & 4.39957500 & 0.63397800 & 0.61343700 \\
\hline C & 3.15378000 & -0.05320300 & 0.12763800 \\
\hline C & 1.95910800 & 0.49849600 & 0.21336000 \\
\hline C & 5.27179800 & -0.33109200 & 1.41759200 \\
\hline S & 5.46750600 & 1.00910600 & -0.93944500 \\
\hline & 0.95927800 & 1.41572400 & 0.51125500 \\
\hline & 5.82014100 & -0.04396800 & 2.45716200 \\
\hline & 5.36028400 & -1.52451500 & 0.82539500 \\
\hline & 6.17467600 & -2.48385900 & 1.50478100 \\
\hline & 4.39834200 & 2.29171500 & -1.75917400 \\
\hline & 3.27356300 & -1.02268000 & -0.35662100 \\
\hline $\mathrm{H}$ & 0.53781000 & 1.43196700 & 1.51874100 \\
\hline $\mathrm{H}$ & 0.81565900 & 2.30219400 & -0.11260300 \\
\hline & 4.14146700 & 1.89109300 & 1.41992800 \\
\hline $\mathrm{H}$ & 3.37248200 & 1.91815300 & -1.86737900 \\
\hline & 4.42158200 & 3.23521400 & -1.20753300 \\
\hline & 4.82921200 & 2.43905000 & -2.75292400 \\
\hline & 5.76157300 & -2.69794300 & 2.49610200 \\
\hline $\mathrm{H}$ & 6.16371200 & -3.38165100 & 0.88424700 \\
\hline & 7.19650500 & -2.10671800 & 1.61580500 \\
\hline & 3.53259600 & 2.60462400 & 0.85322000 \\
\hline & 3.60391600 & 1.63876500 & 2.34323600 \\
\hline & 5.08758700 & 2.36677700 & 1.69416300 \\
\hline & 0.17186700 & -0.27507200 & -0.54656600 \\
\hline & -4.16946700 & -1.91489900 & 3.52710600 \\
\hline & -3.89324500 & -1.10922300 & 2.84336200 \\
\hline & -4.93308400 & -1.04967500 & 1.86828200 \\
\hline & -3.82238100 & -0.16225600 & 3.39613000 \\
\hline & -2.92147200 & -1.32474100 & 2.37681800 \\
\hline C & -4.79765600 & -0.12877200 & 0.91212200 \\
\hline & -5.68044800 & 0.25124500 & -0.06401100 \\
\hline & -3.62803200 & 0.53525600 & 0.82388700 \\
\hline & -4.96692600 & 1.24600000 & -0.81806100 \\
\hline & -7.06334600 & -0.27411400 & -0.26398600 \\
\hline & -3.73088900 & 1.38917300 & -0.25302300 \\
\hline & -5.33124900 & 1.79189100 & -1.68208000 \\
\hline & -7.18896200 & -0.72454300 & -1.25699900 \\
\hline & -7.28966700 & -1.04309200 & 0.48314800 \\
\hline & -7.81623300 & 0.51841000 & -0.16696900 \\
\hline & -2.53645100 & 2.19470700 & -0.54557500 \\
\hline & -2.26616500 & 3.71184300 & 0.55401300 \\
\hline & -2.57904600 & 2.59700700 & -1.56212200 \\
\hline & -1.62778700 & 1.57928200 & -0.45348000 \\
\hline & -2.26823100 & 2.87469500 & 2.21788100 \\
\hline & -1.86991500 & 3.59665000 & 2.93523600 \\
\hline & -3.28383000 & 2.58587300 & 2.50204900 \\
\hline & -1.62917600 & 1.98360400 & 2.19539600 \\
\hline
\end{tabular}

\section{Ru CATALYST}

\subsection{RU-CATALYST Allene MLn-A}

\section{ENERGY $=-1300.51524$}

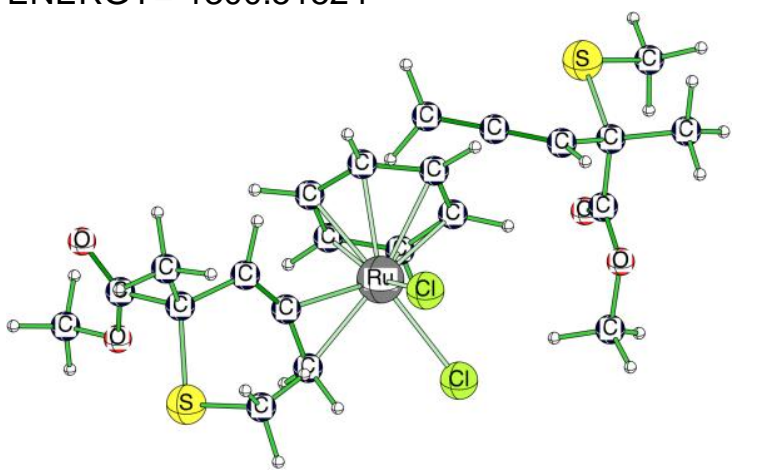

C 4.71954300

1.26117300

$-0.24960800$

C $3.81874800 \quad 2.47859600-0.33105100$

C $2.72175400 \quad 2.57141800 \quad-1.05055700$

C $4.10269700 \quad 0.27125500 \quad 0.75128500$

S $4.78270900 \quad 0.51538700-1.98333400$

C $1.61484100 \quad 2.70636800-1.74378400$

O $3.75080800 \quad-0.86378900 \quad 0.50489700$

$\begin{array}{llll}\text { O } & 4.02105700 & 0.83388900 & 1.95286600\end{array}$

C $3.35411800 \quad 0.05845100 \quad 2.96257300$

C $5.96924500-0.89737900-1.74385600$

H $4.09681200 \quad 3.31628700 \quad 0.31399600$

H $1.61605500 \quad 3.11684400 \quad-2.75327800$

H $0.66737800 \quad 2.40987300-1.28522800$

C $6.11144600 \quad 1.67432900 \quad 0.22315900$

H $5.60663200-1.55705800-0.95181900$

H $5.98712200 \quad-1.43780400-2.69409300$

H $6.97516100-0.52923400-1.52242300$

H $3.84160900-0.91546700 \quad 3.07846900$

H $3.43765300 \quad 0.64103700 \quad 3.88225900$

H $2.30027800 \quad-0.08619600 \quad 2.69014200$

H $6.56652700 \quad 2.34709600-0.51297100$

H $6.04061800 \quad 2.19819200 \quad 1.18428100$

H $6.76871100 \quad 0.80778800 \quad 0.36401300$

Cl $0.31845800 \quad-2.04064800 \quad 2.44177700$

Cl $0.35264800 \quad 1.03305100 \quad 1.09256800$

C $0.13988400 \quad-0.73907100 \quad-1.92732100$

C $1.37195800 \quad-1.18978600-1.35985400$

C $1.39666500-2.39050000-0.64389800$

C $0.20520000-3.18391000-0.51640200$

C $-0.97607300-2.81861000-1.19623000$

C $-1.00732000-1.57747500-1.89183000$

H $0.09078900 \quad 0.23830900 \quad-2.40360900$

H $2.26265600 \quad-0.55874500-1.38445900$

H $2.28643000 \quad-2.65241700-0.07868400$

H $0.21144600 \quad-4.05792800 \quad 0.12881500$

H $-1.87660100-3.42001500-1.10269900$

H $-1.94307700 \quad-1.22597800 \quad-2.32068200$

Ru -0.35768300 $-1.15711100 \quad 0.20722900$

C $-4.10197000 \quad 1.46424300 \quad-0.33343000$

C $-2.72440300 \quad 0.84665300 \quad-0.51978800$

C $-2.24436500 \quad-0.13651300 \quad 0.22370500$

C $-5.05626800 \quad 0.60436800-1.16292800$

S $-4.69370500 \quad 1.42243700 \quad 1.46918500$ 


$\begin{array}{rrrr}\text { C } & -2.27040000 & -1.00053600 & 1.29386600 \\ \text { O } & -5.37712000 & 0.86593200 & -2.30278700 \\ \text { O } & -5.43266400 & -0.50437700 & -0.52155800 \\ \text { C } & -6.26878300 & -1.38772900 & -1.26983900 \\ \text { C } & -3.39016600 & 2.50602000 & 2.23716200 \\ \text { H } & -2.13019100 & 1.27878900 & -1.33143100 \\ \text { H } & -2.03553600 & -0.62173200 & 2.28997700 \\ \text { H } & -2.79908000 & -1.95356400 & 1.24474100 \\ \text { C } & -4.09325800 & 2.88812900 & -0.87172500 \\ \text { H } & -2.39409600 & 2.18438900 & 1.90892900 \\ \text { H } & -3.55697800 & 3.55974300 & 1.99808100 \\ \text { H } & -3.47783000 & 2.35814000 & 3.31681800 \\ \text { H } & -5.75027100 & -1.74082200 & -2.16864000 \\ \text { H } & -6.49311900 & -2.22428500 & -0.60547200 \\ \text { H } & -7.19181900 & -0.88059000 & -1.56874900 \\ \text { H } & -3.34119700 & 3.48878000 & -0.34697500 \\ \text { H } & -3.84101100 & 2.88434000 & -1.93941800 \\ \text { H } & -5.07511700 & 3.35886500 & -0.75063800\end{array}$

\subsection{RU-CATALYST TS1}

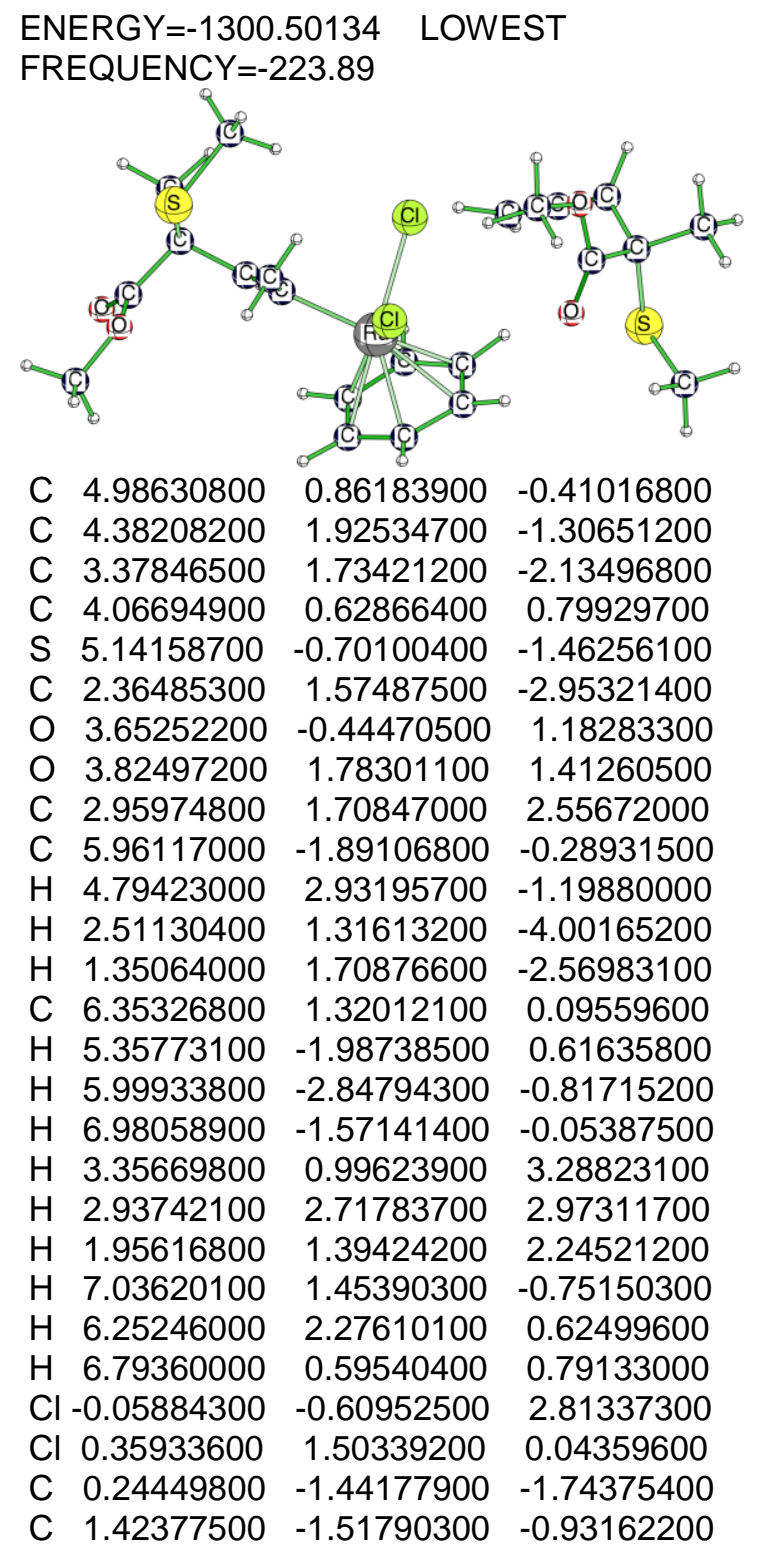
C $1.43629100-2.30169800$
0.23451200
C $0.24092900-2.97852200$
0.63741000
C $-0.91532600-2.96893700$
$-0.18228500$
C $-0.90680400-2.18523800-1.37711300$
H $0.21932400-0.77406500-2.60228300$
H $2.28328500-0.88737100-1.16004800$
H $2.28939600 \quad-2.23361600 \quad 0.90395800$
H $0.19980100-3.45299300 \quad 1.61440700$
H $-1.82674000 \quad-3.46101000 \quad 0.14588200$
H $-1.82223300 \quad-2.08391500-1.95583200$
Ru $-0.33682500 \quad-0.88222200 \quad 0.29211400$
C $-4.33386000 \quad 1.02569200 \quad-0.82201300$
C $-2.98559900 \quad 0.33128800 \quad-0.98752000$
$\begin{array}{lll}\text { C }-2.25925000 & -0.00129400 & 0.07677700\end{array}$
C $-5.38808400 \quad-0.07453800 \quad-0.94215400$
S $-4.46153500 \quad 1.75972400 \quad 0.92389400$
C $-2.50387300 \quad 0.26324500 \quad 1.42542900$
$\begin{array}{llll}\text { O } & -5.93039300 & -0.36663900 & -1.98507100\end{array}$
$\begin{array}{llll}O & -5.57078700 & -0.72801800 & 0.20816500\end{array}$
C $-6.46835300 \quad-1.83971300 \quad 0.14841100$
C $-3.31580000 \quad 3.21549400 \quad 0.74757500$
H $-2.68489900 \quad 0.13472200 \quad-2.01984800$
H $-1.95900700 \quad 1.06318200 \quad 1.92669700$
H $-2.96650400-0.48199900 \quad 2.07303100$
C $-4.54090000 \quad 2.08050400 \quad-1.89669500$
H $-2.38948700 \quad 2.89822700 \quad 0.25306000$
H $-3.80836700 \quad 4.01789500 \quad 0.19419100$
H $-3.09543300 \quad 3.54678100 \quad 1.76563800$
H $-6.09885900-2.58794700 \quad-0.56102900$
H $-6.50219600 \quad-2.25297500 \quad 1.15799900$
H $-7.46512600-1.51166300 \quad-0.16357700$
H $-3.72510200 \quad 2.81140100-1.88014800$
H $-4.53693500 \quad 1.59209100 \quad-2.87798900$
H $-5.499660002 .59839600 \quad-1.77798400$

\subsection{RU-CATALYST S-Cycle}

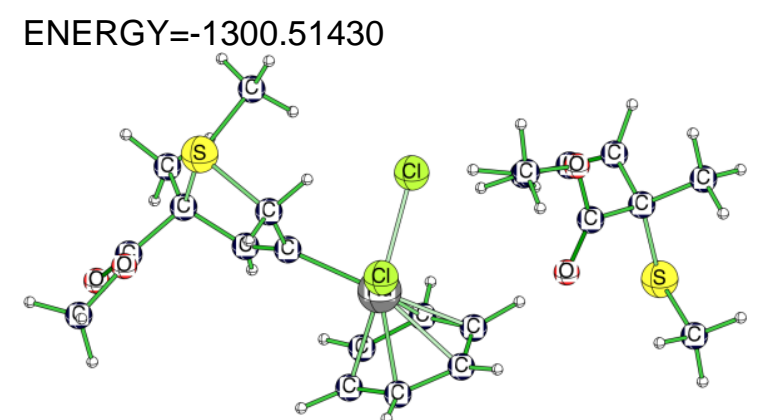
C 5.04423700
$0.87872000-0.12493700$
C 4.47906100
$1.92006300-1.07277400$
C 3.55345000
$1.69316300-1.97980900$
C 3.99165700
$0.57204900 \quad 0.95218700$
S 5.41377900
$-0.65162600$
$-1.16817600$
C 2.61101900
$1.50177300-2.87259400$
O 3.49732900
$-0.51074200$
1.18109100
O 3.70631200
1.68063400
1.63243700
C 2.66480500
$1.54528600 \quad 2.61238800$
C 6.12765500
$-1.81554200$
0.09695000
H 4.84683100
2.94028100
$-0.93575400$
H 2.84440300 


$\begin{array}{rrrr}\text { H } & 1.56723500 & 1.59810000 & -2.55838300 \\ \text { C } & 6.31426500 & 1.41016900 & 0.53565400 \\ \text { H } & 5.40949900 & -1.96496200 & 0.90729300 \\ \text { H } & 6.29596000 & -2.76005000 & -0.42763700 \\ \text { H } & 7.08336500 & -1.44376500 & 0.47858300 \\ \text { H } & 2.94969500 & 0.80816100 & 3.37100700 \\ \text { H } & 2.55255700 & 2.53515300 & 3.06044300 \\ \text { H } & 1.73380500 & 1.22703700 & 2.12818600 \\ \text { H } & 7.07607900 & 1.60219800 & -0.22924900 \\ \text { H } & 6.09913300 & 2.34686700 & 1.06490400 \\ \text { H } & 6.72112200 & 0.69925200 & 1.26521800 \\ \text { Cl }-0.32936600 & -0.62371000 & 2.57644500 \\ \text { Cl } & 0.30349100 & 1.53612300 & -0.20115100 \\ \text { C } & 0.52823600 & -1.41543400 & -1.87763700 \\ \text { C } & 1.57619700 & -1.65316200 & -0.92403400 \\ \text { C } & 1.32640000 & -2.45082800 & 0.19494800 \\ \text { C } & 0.01872400 & -3.01672900 & 0.38454100 \\ \text { C }-0.98663900 & -2.88212500 & -0.60770900 \\ \text { C } & -0.72420300 & -2.06860800 & -1.75499900 \\ \text { H } & 0.69224700 & -0.70868300 & -2.68844200 \\ \text { H } & 2.52133300 & -1.11571900 & -1.00249900 \\ \text { H } & 2.06736700 & -2.50787900 & 0.98714100 \\ \text { H }-0.21426400 & -3.52233400 & 1.31836100 \\ \text { H }-1.97609100 & -3.30356200 & -0.44670700 \\ \text { H }-1.50546900 & -1.88716700 & -2.48907300 \\ \text { Ru }-0.35889300 & -0.89763700 & 0.05393900 \\ \text { C } & -4.39770600 & 0.83120200 & -0.85766800 \\ \text { C } & -3.12073500 & 0.02699900 & -0.98454500 \\ \text { C }-2.25125800 & -0.04209100 & 0.04169100 \\ \text { C }-5.52709000 & -0.14081700 & -0.52158600 \\ \text { S }-4.06588600 & 1.91724800 & 0.68610300 \\ \text { C } & -2.63983600 & 0.73854600 & 1.25195000 \\ \text { O } & -6.19036900 & -0.68501700 & -1.37473900 \\ \text { O }-5.62011100 & -0.38174600 & 0.78672500 \\ \text { C }-6.55679500 & -1.39814500 & 1.16558400 \\ \text { C }-2.99370800 & 3.25949500 & -0.02407400 \\ \text { H } & -2.98750900 & -0.45689100 & -1.95304900 \\ \text { H }-1.85317900 & 1.40096400 & 1.62533400 \\ \text { H }-3.05855800 & 0.14156600 & 2.06649600 \\ \text { C }-4.73039400 & 1.66021300 & -2.08188400 \\ \text { H }-2.14855500 & 2.79421300 & -0.54635700 \\ \text { H }-3.60921900 & 3.88348500 & -0.67438200 \\ \text { H }-2.64046700 & 3.83574500 & 0.83417100 \\ \text { H } & -6.27886300 & -2.35314400 & 0.70857600 \\ \text { H }-6.50294700 & -1.46430400 & 2.25312200 \\ \text { H }-7.56645400 & -1.12215900 & 0.84566800 \\ \text { H }-3.86144400 & 2.24312600 & -2.40528300 \\ \text { H }-4.99537600 & 0.96842700 & -2.88923700 \\ \text { H }-5.58667300 & 2.32549700 & -1.91671900\end{array}$

\subsection{RU-CATALYST TS2}

ENERGY $=-1300.49902 \quad$ LOWEST FREQUENCY $=-278.43$

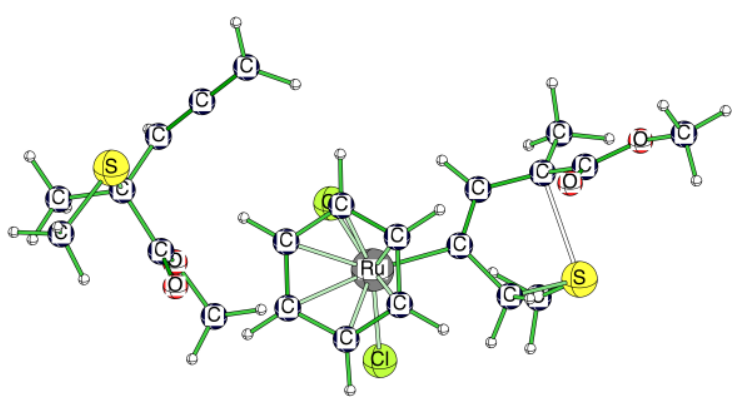

C -4.95166100

C -4.18845500

C -3.26509600

C -4.13417500

$0.91157300-0.68461800$ $2.04390000-1.34315600$

S -5.18306100 $2.77636500-0.76063400$

C -2.32660000

O -3.82912200 $-0.38361700$ $-0.81535300$

O -3.83663700 $1.37844000 \quad 1.13097900$

C -3.00975400 $3.50290200-0.20056000$

C -6.24653700

H -4.39413700

H -2.55027100

H -1.29597300

C -6.29756500

H -5.76112800

H -6.32802500

$-1.12632500 \quad 0.09462200$

$-0.60902900-2.09116900$

$-1.75571000-2.34339900$

$-0.01743100 \quad 1.75109100$

$2.19473400-2.40606500$

$4.44778000 \quad 0.29375900$

$3.14182600-0.22849000$

$0.72069500-1.38315800$

$-0.97459200 \quad 1.54532900$

H -7.24609500

0.12967000

2.83154400

H -3.47833900

$0.02319900 \quad 1.30866400$

H -2.92400200

$-2.65894700-1.93746300$

H -2.01866600

$-1.82346100$

$-3.42977400$

H -6.90817700

$-1.61273300$

$-1.89366200$

H -6.13728200

$1.62347700-1.26387700$

H -6.85065600

$0.54097400-2.45384800$

Cl 0.49730300

$-0.13589800-0.97883200$

Cl -0.54900700

$-2.69265500-1.03756100$

C -1.48940500

$0.62861800-1.26192100$

C -1.45294500

$-0.18104900$

1.85722500

C -0.21810500

$-1.58115100$

1.85787500

C 0.91509400

$-2.23904200$

2.15445800

C 0.85997100

C -0.32380700

$-1.51161300$

2.60759100

H -2.38585800

$-0.08201300$

2.61382200

0.58426700

2.20338800

H -2.30348100

0.32486900

1.49443000

H -0.13736300

$-2.13399500$

1.47073200

H 1.83700300

$-3.31497600$

2.01890500

H 1.75735800

$-2.03301600$

2.85332800

H -0.34442400

0.49447400

2.83370100

Ru 0.34096400

1.66836500

2.12761200

C 3.93436400

$-0.77781300$

0.59934000

C 2.56970600

1.27334000

$-0.77951000$

C 2.18310600

0.92359500

$-0.56461300$

C 4.82865100

$-0.26622800$

$-0.00071900$

S 4.71435700

1.40173100

0.44047200

C 3.24360900

$-0.97202700$

$-1.01463500$

O 4.51544400

$-1.33121800$

0.13279200

O 6.00271200

C 6.92183900

1.05522000

1.56001600

1.95193200

0.12723000

$2.09240700 \quad 1.21448800$

C 3.84400300

$-1.35900000$

$-2.60652800$

H 1.80661900

1.53771800

$-1.05421800$

H 2.84670600

$-2.30761000$

$-0.16962500$

H $3.66056800 \quad-1.38323100 \quad 1.14629300$ 

C 4.28638100
$2.10681100-1.97171000$
H 3.67427300
$-2.43749800-2.64887700$
H 2.87749800
$-0.83661200-2.60418400$
H 4.48433400
$-1.02895000$
$-3.42683500$
H 7.18720100
1.10851800
1.61599200
H 7.80334800
2.58503600
0.80059500
H 6.48074800
2.69841700
2.01229200
H 3.63917300
$1.85921900-2.81974800$
H 4.13303000
$3.16710300-1.72436200$
H 5.33695000
$1.99447200-2.25564900$

3.5 RU-CATALYST Carbenoid

This structure was obtained as a shoulder ENERGY $=-1300.52238$

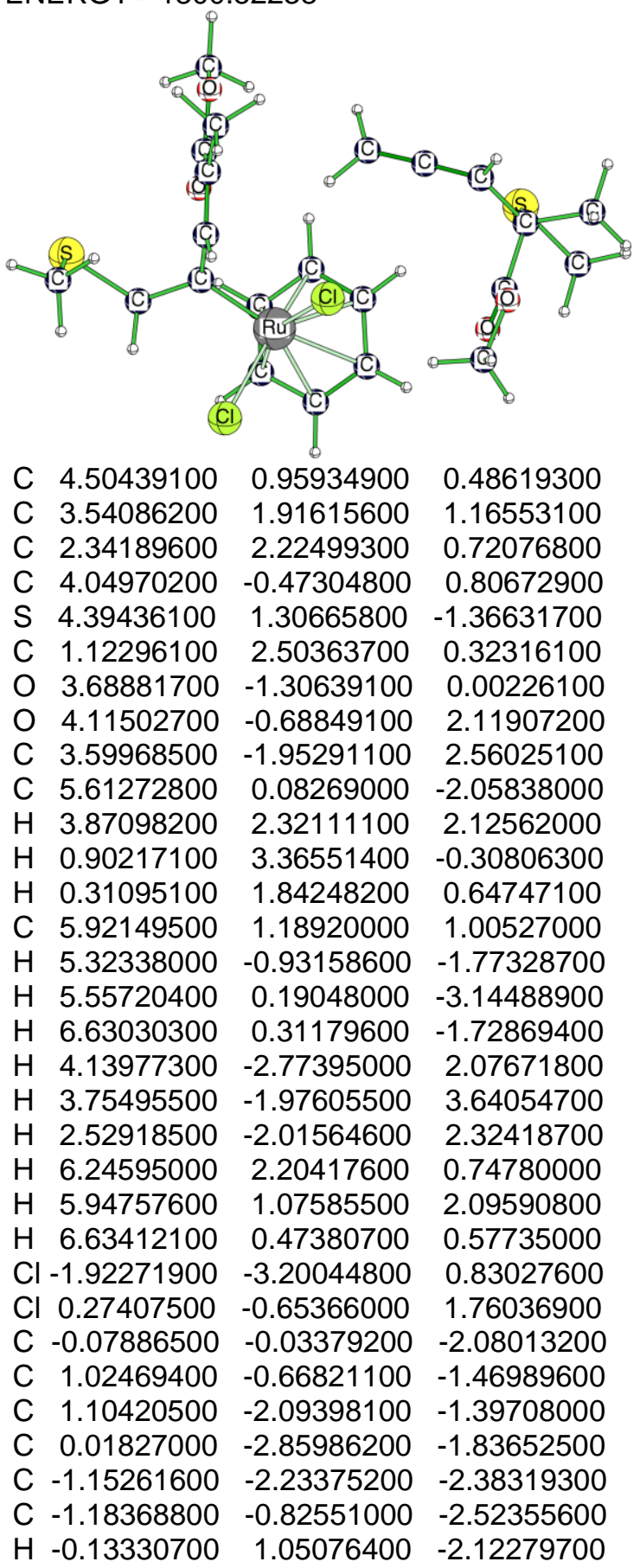

H $\quad 1.82610600 \quad-0.07050500 \quad-1.03787100$

H $1.95076700 \quad-2.54452000-0.88712300$

H $0.00210700 \quad-3.93110000-1.65198900$

H $-2.01499700-2.83576000-2.65889200$

H $-2.07338900 \quad-0.33235700 \quad-2.90840800$

Ru -0.86411000 $-1.29570800 \quad-0.39048800$

C $-2.29075900 \quad 2.04335600 \quad 1.09536100$

C $-2.32982100 \quad 0.70361400 \quad 1.24050900$

C $-2.43530300 \quad-0.25298200 \quad 0.15520100$

C $-2.35873500 \quad 2.48339300 \quad-0.30602800$

S $-5.30254900 \quad 0.27526400 \quad 0.12597100$

C $-3.77596000-0.71217400-0.31153000$

O $-2.47845800 \quad 1.69898600 \quad-1.24311900$

O $-2.25388400 \quad 3.80185200-0.46201100$

C $-2.27138700 \quad 4.26389100 \quad-1.81404500$

C $-5.48724500 \quad-0.26233000 \quad 1.89873200$

H $-2.21826300 \quad 0.24264400 \quad 2.22672800$

H $-3.89041900-1.73017900 \quad 0.10177600$

H $-3.78258700 \quad-0.82397300-1.40144100$

C $-2.11972800 \quad 3.02126200 \quad 2.21176700$

H $-4.71860800 \quad 0.20139500 \quad 2.52527300$

H $-6.47478200 \quad 0.06788200 \quad 2.23013000$

H $-5.42365000-1.35293900 \quad 1.96653300$

H $-3.19646000 \quad 3.95731000-2.31283700$

H $-2.20566000 \quad 5.35194700 \quad-1.76301200$

H $-1.41495300 \quad 3.85725300 \quad-2.36573900$

H $-2.08680800 \quad 2.50163600 \quad 3.17486900$

H $-1.18571700 \quad 3.58777900 \quad 2.09684700$

H $-2.93984400 \quad 3.74941100 \quad 2.22907100$

\subsection{RU-CATALYST Cycle-O MLn-F}

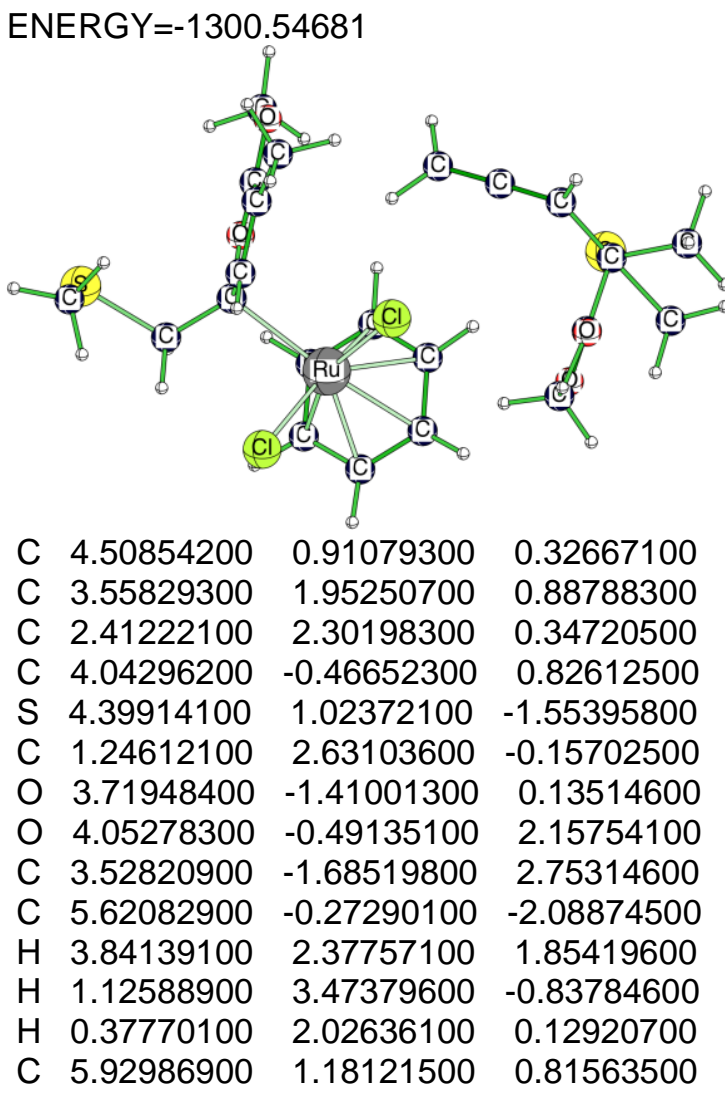


H $5.33205700-1.24486600 \quad-1.68266300$

H $5.56509600-0.29799500-3.18029000$

H $6.63803200 \quad-0.00357800 \quad-1.78969400$

H $4.12861900 \quad-2.55380800 \quad 2.46133400$

H $3.58350200-1.52789700 \quad 3.83199700$

H $2.48555200 \quad-1.82230800 \quad 2.44095200$

H $6.26930100 \quad 2.15601200 \quad 0.44649500$

H $5.95391900 \quad 1.18970400 \quad 1.91240900$

H $6.63077900 \quad 0.41121100 \quad 0.47088500$

Cl $-2.06349500 \quad-2.68716500 \quad 1.32420900$

Cl $0.37630200 \quad-0.23358000 \quad 1.67331200$

C $0.02583500 \quad-0.34125900-2.14459100$

C $1.05183400-0.96779900-1.39776700$

C $0.95548400-2.34184500-1.02227500$

C $-0.21880100 \quad-3.05959300-1.34136700$

C $-1.27934200 \quad-2.44231900-2.07920000$

C $-1.15126300 \quad-1.08233900-2.46766400$

H $0.09482700 \quad 0.71960300 \quad-2.37507200$

H $1.89901500-0.37992800-1.04763400$

H $1.72013800-2.76734100-0.37780000$

H $-0.36877000-4.05530600-0.93121600$

H $-2.20705100-2.98124200-2.25813200$

H $-1.98146800-0.58080300-2.96207800$

Ru $-0.84959900 \quad-1.22224300-0.30664800$

C $-2.16623800 \quad 1.96618400 \quad 1.26558400$

C $-2.46973900 \quad 0.61666700 \quad 1.25657300$

C $-2.63585100 \quad 0.14746700-0.08607200$

C $-2.20563100 \quad 2.32644400 \quad-0.08947400$

S $-5.47381700 \quad 0.37480200 \quad-0.29303200$

C $-3.86955500 \quad-0.58633500-0.53244100$

$\begin{array}{llll}\text { O } & -2.46668300 & 1.33208000 & -0.90440600\end{array}$

$\begin{array}{llll}\text { O } & -1.95888500 & 3.51944500 & -0.56521300\end{array}$

C $-2.06610200 \quad 3.70493200-1.98236800$

C $-5.68750600 \quad 0.13163500 \quad 1.53982000$

H $-2.54207900 \quad-0.04025100 \quad 2.11849600$

H $-3.95171300 \quad-1.53330500 \quad 0.01175100$

H $-3.83951700 \quad-0.79523300-1.60751100$

C $-1.76595600 \quad 2.85617600 \quad 2.39161600$

H $-5.04544300 \quad 0.81753100 \quad 2.10121100$

H $-6.73527600 \quad 0.33743900 \quad 1.77229100$

H $-5.45152000 \quad-0.90595900 \quad 1.79966000$

H $-3.04924200 \quad 3.37961300-2.33747500$

H $-1.93341900 \quad 4.77392300-2.15254700$

H $-1.27870400 \quad 3.14217600 \quad-2.49812700$

H $-1.91391500 \quad 2.34630700 \quad 3.34849800$

H $-0.70198100 \quad 3.11946300 \quad 2.31778200$

H $-2.34512400 \quad 3.78721400 \quad 2.39523400$

\subsection{RU-REGENERATION TSx}

ENERGY $=-1300.52526 \quad$ LOWEST

FREQUENCY $=-60.75$

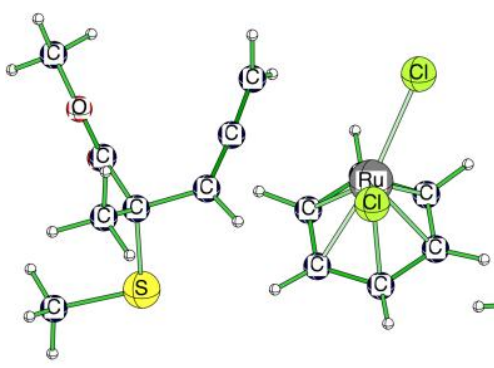

C 3.82689100

$-1.46323200$

C $3.52423600-0.07522800$

C $4.26861300 \quad 0.33378700$

(c)

C 4.73537200

$-1.78129400$

1.12694600

S 5.87423800

2.65695200

C 4.43886100

1.63636600

O 5.01838900

$-0.71630700$

0.90089100

$-0.16974600$

0.15618600

O 5.30160600

$-2.95248800$

$-0.14118100$

C 6.72330300

$-2.90997700$

C 5.13261200

2.98120300

$-0.83407400$

H 2.81826400

0.53697400

$-0.64121600$

H 4.67118000

1.51960200

$-0.15713400$

$-0.28047800$

H 3.52514200

2.23503100

1.53344000

1.45553000

C 3.25893700

$-2.37232800$

$-1.89866900$

H 5.03034700

2.04232400

$-0.71852300$

H 5.81175300

3.65340500

2.16417600

2.08719300

H 4.15219400

3.45648800

2.06387200

H 7.17821100

$-2.51951700$

1.41834400

H 7.05334900

$-3.93821000$

0.63999200

H 7.02488100

$-2.28414300$

$-0.44813800$

H 2.16418600

$-2.42793900$

$-1.12919700$

H 3.67167100

$-3.38262600$

2.08127500

H 3.47847400

$-2.00977000$

2.06361500

Cl 0.93639200

2.33283300

3.17601200

Cl -0.02213100

$-0.32730300$

0.03711200

C 0.15737100

$-2.00110300$

2.01732200

C 1.28052000

$-1.24798700$

$-0.85549200$

C 1.09024900

$-0.13557900$

$-1.31853800$

C -0.21837500

0.25712300

$-2.19812600$

C -1.33155000

$-0.44742000$

$-2.57188900$

C -1.14858100

$-1.60184300$

$-2.03091100$

H 0.30594300

$-2.77809000$

$-1.21008900$

H 2.27336600

$-1.46537700$

$-0.10928400$

H 1.94438500

0.48606100

$-0.92692100$

H -0.37289700

1.17588500

$-2.45874600$

H -2.34186500

$-0.06883600$

$-3.13148200$

H -2.01677400

$-2.07807600$

$-2.18684700$

$\mathrm{Ru}-0.04142400$

0.13079500

C -4.85900000

$-0$.

$-0.75581900$

C -3.41546700

$-0.42248600$

$-0.36683600$

C -2.90441400

0.01064000

0.78953700

C -5.54345800

1.08839600

0.96659900

S -4.74757600

C -2.47792300

0.62611700

0.45525400

$-2.07992900$

$-0.09215800$

2.23565800

$-0.12370900$

O -5.59872800

0.60912300

$-0.02633200$

O -6.02843800

C -6.55537600

1.62050400

$-1.30141900$

C -6.54847900

2.72992800

0.65871100

$-2.46845000$

$-0.07191500$

H -2.77564500

$-0.68649800$

$-0.37784500$

H -2.48113600

H -2.07427200
3.13168300

2.33370500
0.59412100

$-1.03441800$ 

C $-5.52592100-0.60237100$
H $-7.05007000-2.63338200$
2.14990500
H $-7.02721900-1.66167700$
0.57982100
H $-6.58066700-3.38884900$
$-0.94028300$
H -7.40777100
2.41788800
$-0.96613100$
H -6.87005300
3.46107700
$-0.68471400$
H -5.78292200
3.15378400
H -5.48650100
0.33455800
H -6.57801200
$-0.89691200$
0.67503500
$-0.72361300$
2.71650900
2.05083800
2.71508600

\subsection{RU-REGENERATION Furane}

ENERGY $=-1300.54093$
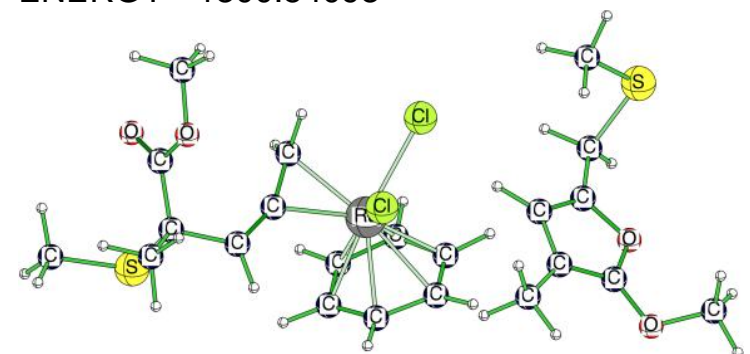

C $3.80613600-1.18934600$

1.44053200

C $3.36358700-0.02688000$

0.71679300

C 4.32184500

0.25947300

$-0.21347300$

C 5.00735800

$-1.51193600$

0.87295800

S 5.54656200

2.78116700

$-0.56673900$

C 4.46620200

1.35554000

$-1.18527200$

O 5.34313200

$-0.65603900$

$-0.12657600$

O 5.82462400

$-2.54584300$

1.10363800

C 7.20517700

$-2.20490400$

1.21928200

C 4.41670100

3.34575500

0.79956800

H 2.43724900

0.52336100

0.87203800

H 4.94858900

1.02605000

$-2.11253700$

H 3.47777700

1.77857700

$-1.40963300$

C 3.11437300

$-1.89842500$

2.55691200

H 4.34679200

2.57556300

H 4.84752100

4.25866500

1.57425700

H 3.42110400

3.55448300

1.21842000

0.39231600

H 7.35202200

$-1.44096900$

1.99490800

H $7.72973300-3.12157000$

1.50026200

H $7.60021900-1.83307100$

0.26614200

H $2.14349900-2.30593800$

2.23874500

H 3.72844700

$-2.72581100$

2.93024300

H 2.90348400

$-1.21674000$

3.38982400

Cl 0.74920300

2.02618400

$-0.93415900$

$\mathrm{Cl}-0.10154300$

0.05712100

1.68070400

C -0.23129000

$-2.36499700$

$-0.67279300$

C $1.08845700-1.86372600-0.92723100$

C $1.29933400 \quad-0.99251700 \quad-2.00025600$

C $0.20420900-0.58001000-2.83251900$

C $-1.06892900-1.16899100-2.65746100$

C $-1.27862600 \quad-2.07975300-1.58156700$

H $-0.42223000 \quad-2.96939200 \quad 0.21101600$

H $1.90070600-2.06324900-0.22988900$

H $2.27074400-0.51403100-2.10950800$

H $0.35600300 \quad 0.18556800 \quad-3.58812300$

H $-1.90888000-0.86250300-3.27681100$

H $-2.27978200 \quad-2.46092900-1.39324800$
Ru $-0.45332100 \quad-0.15215900-0.75380300$

C $-4.74206600 \quad-0.43068900 \quad 0.80798600$

C $-3.28561600 \quad-0.73946500 \quad 0.56443100$

C $-2.49699300 \quad 0.06969800 \quad-0.12095200$

C $-4.98451400 \quad 1.08259700 \quad 0.77702100$

S $-5.60476800-1.28157000 \quad-0.64730700$

C $-2.16781300 \quad 1.24126900 \quad-0.76285100$

$\begin{array}{llll}\text { O } & -5.61522000 & 1.69521200 & -0.05472700\end{array}$

$\begin{array}{llll}\text { O } & -4.38960500 & 1.65319400 & 1.82935900\end{array}$

C $-4.49946500 \quad 3.07585000 \quad 1.90183500$

$\begin{array}{lll}\text { C }-7.39934700 & -1.02354500 & -0.22593800\end{array}$

H $-2.92459000 \quad-1.69792800 \quad 0.94647100$

H $-1.87435200 \quad 2.10793100 \quad-0.16789600$

H $-2.456739001 .42988900-1.79765300$

C $-5.19401400 \quad-1.01667900 \quad 2.14401400$

H $-7.67743700 \quad-1.58725100 \quad 0.66916000$

H $-7.59106200 \quad 0.04531900-0.09824600$

H -7.96850900 $-1.39680400-1.08128900$

H $-5.54544500 \quad 3.37422800 \quad 2.03179600$

$\begin{array}{llll}H & -3.90435900 & 3.37888900 & 2.76497000\end{array}$

H $-4.10913300 \quad 3.53664600 \quad 0.98756300$

H $-4.58099300-0.60987400 \quad 2.95625300$

H $-6.24372200 \quad-0.77851000 \quad 2.35537700$

H $-5.08114500 \quad-2.10745100 \quad 2.12622700$

\section{Coordination of the Sulfur on the Ru CATALYST}

\subsection{So-complexe bimolecular}

ENERGY $=-1300.52943$
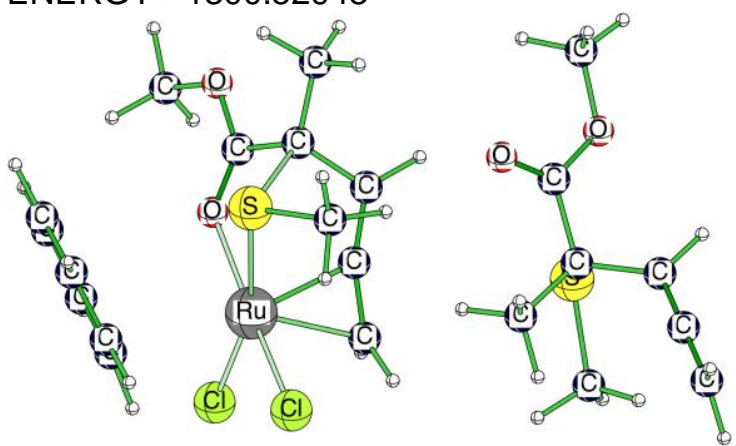

$\begin{array}{llll}\text { C } & -0.70027900 & 2.03379900 & 0.17952300\end{array}$

C $\quad 0.40150400 \quad 1.36896500-0.59438500$

C $\quad 0.16122600 \quad 0.10679100 \quad-0.94821300$

C $\quad-1.86653000 \quad 2.08766700-0.79429800$

S $\quad-1.36540700 \quad 0.69223300 \quad 1.44180300$

C $\quad 0.27821400 \quad-1.11123900-1.58712800$

$\begin{array}{llll}\text { O } & -2.35644000 & 1.07172700 & -1.29912000\end{array}$

$\begin{array}{llll}O & -2.27435200 & 3.29818000 & -1.10084300\end{array}$

C $\quad-3.29910400 \quad 3.38426900-2.10732900$

$\begin{array}{llll}\text { C } & 0.14514300 & 0.31321300 & 2.44786800\end{array}$

H $\quad 1.29333100 \quad 1.93246300 \quad-0.87678800$

H $\quad 0.83225100 \quad-1.93494800-1.13288000$

H $\quad 0.08880600 \quad-1.18634800-2.65861800$

C $\quad-0.33069600 \quad 3.33227000 \quad 0.85240600$

$\begin{array}{llll}\text { H } & 0.09003000 & -0.75976000 & 2.65850600\end{array}$ 


$\begin{array}{cccc}\mathrm{H} & 1.03945700 & 0.53753900 & 1.85666200 \\ \mathrm{H} & 0.11376000 & 0.90990900 & 3.36163700 \\ \mathrm{H} & -3.52528300 & 4.44633300 & -2.20767400 \\ \mathrm{H} & -2.92891500 & 2.97372100 & -3.05154000 \\ \mathrm{H} & -4.18551400 & 2.82558100 & -1.79213000 \\ \mathrm{H} & 0.51072000 & 3.16474500 & 1.53688300 \\ \mathrm{H} & -0.01532500 & 4.06480300 & 0.09825900 \\ \mathrm{H} & -1.17084800 & 3.75540200 & 1.41342500 \\ \mathrm{Cl} & -2.42842100 & -2.07657500 & -2.42131100 \\ \mathrm{Cl} & -0.70151400 & -2.74828700 & 0.77513500 \\ \mathrm{C} & -3.78934800 & -1.68549200 & 2.06732200 \\ \mathrm{C} & -4.31287700 & -0.62016200 & 2.79012800 \\ \mathrm{C} & -4.91479700 & 0.45686000 & 2.11702500 \\ \mathrm{C} & -4.99090100 & 0.46130800 & 0.72697800 \\ \mathrm{C} & -4.47843900 & -0.62138100 & -0.00688300 \\ \mathrm{C} & -3.87449700 & -1.69509300 & 0.66081700 \\ \mathrm{H} & -3.31869400 & -2.52323600 & 2.57838800 \\ \mathrm{H} & -4.25603000 & -0.61713900 & 3.87759400 \\ \mathrm{H} & -5.32406700 & 1.29042300 & 2.68668800 \\ \mathrm{H} & -5.46830200 & 1.29161400 & 0.20741700 \\ \mathrm{H} & -4.56668200 & -0.65080900 & -1.09201500 \\ \mathrm{H} & -3.55483300 & -2.57343300 & 0.09996500 \\ \mathrm{Ru} & -1.56862700 & -0.83230000 & -0.48234500 \\ \mathrm{C} & 4.17792500 & -0.12728600 & 0.15770500 \\ \mathrm{C} & 5.65265800 & -0.35212900 & 0.38093200 \\ \mathrm{C} & 6.14682500 & -1.40017200 & 1.00744800 \\ \mathrm{C} & 3.81751100 & 1.28024800 & 0.62748800 \\ \mathrm{~S} & 3.86502200 & -0.09408800 & -1.74046700 \\ \mathrm{C} & 6.63808000 & -2.44112400 & 1.63771900 \\ \mathrm{O} & 3.01307900 & 1.52993700 & 1.50116300 \\ \mathrm{O} & 4.51045200 & 2.21756200 & -0.02301600 \\ \mathrm{C} & 4.24227500 & 3.56464900 & 0.37136200 \\ \mathrm{C} & 4.12711400 & -1.89342800 & -2.12150800 \\ \mathrm{H} & 6.32798700 & 0.39645300 & -0.03862000 \\ \mathrm{H} & 6.81771200 & -2.41497700 & 2.71246400 \\ \mathrm{H} & 6.88369800 & -3.36059700 & 1.10624700 \\ \mathrm{C} & 3.28770300 & -1.14770000 & 0.83952900 \\ \mathrm{H} & 5.05269300 & -2.23804700 & -1.64669300 \\ \mathrm{H} & 3.27735000 & -2.50098900 & -1.79887700 \\ \mathrm{H} & 4.23334800 & -1.95786700 & -3.20733600 \\ \mathrm{H} & 4.88580100 & 4.19285600 & -0.24695600 \\ \mathrm{H} & 3.18827600 & 3.81215800 & 0.19767200 \\ \mathrm{H} & 4.47101100 & 3.70595800 & 1.43279300 \\ \mathrm{H} & 3.55870100 & -2.16792500 & 0.54723900 \\ \mathrm{H} & 3.39519200 & -1.07109400 & 1.92891800 \\ \mathrm{H} & 2.23483900 & -0.97753600 & 0.58364500\end{array}$

\subsection{TSso1 bimolecular}

ENERGY $=-1300.50397 \quad$ LOWEST FREQUENCY $=-24.86$
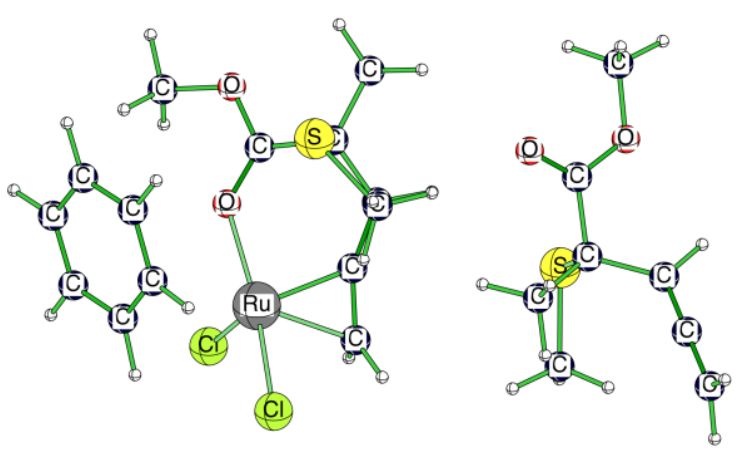

C $\quad-0.35399000 \quad 2.00063500$

$\begin{array}{llll}0.46827600 & 0.83184000 & -0.58173100\end{array}$

C $0.00281200 \quad-0.41045700 \quad-0.65777900$

C $\quad-1.74570000 \quad 1.86336800 \quad-0.73052800$

S $\quad-0.70798600 \quad 2.02974600 \quad 1.76736200$

C $0.06645800-1.76361000-0.95924900$

O $\quad-2.36433800 \quad 0.80508100 \quad-0.87379500$

O $\quad-2.27156800 \quad 3.00801300 \quad-1.10698200$

C $\quad-3.57334600 \quad 2.95716500 \quad-1.71317500$

C $\quad 0.48078900 \quad 0.76344100 \quad 2.43523000$

H $\quad 1.50679700 \quad 1.02995700 \quad-0.86726400$

H $\quad 0.56674200 \quad-2.46236700 \quad-0.28548500$

H $\quad-0.06485900 \quad-2.09640600 \quad-1.99087200$

C $\quad 0.33204800 \quad 3.31031200 \quad-0.50169500$

H $\quad 0.20907900 \quad-0.25058200 \quad 2.11966700$

H $\quad 1.50198600 \quad 1.00879200 \quad 2.12844100$

H $\quad 0.38386900 \quad 0.84245300 \quad 3.52221800$

H $\quad-3.80427200 \quad 3.98443600 \quad-1.99822600$

H $\quad-3.55412600 \quad 2.30229100 \quad-2.58960000$

H $\quad-4.30508700 \quad 2.57561800 \quad-0.99332200$

H $\quad 1.31258600 \quad 3.32818200 \quad-0.01071500$

H $\quad 0.47643300 \quad 3.38481400 \quad-1.58837900$

H $\quad-0.24378000 \quad 4.17594200 \quad-0.16196300$

Cl $\quad-2.70679100 \quad-1.88186800 \quad-2.20012600$

Cl $-1.48031300 \quad-3.10734000 \quad 1.21161200$

C $\quad-3.76559500 \quad-0.64925400 \quad 1.80138800$

C $\quad-4.13586100 \quad 0.68764300 \quad 2.02634900$

C $\quad \begin{array}{llll}-5.07440500 & 1.29254900 & 1.19678900\end{array}$

C $\quad \begin{array}{llll}-5.63952000 & 0.57998600 & 0.12468700\end{array}$

C $\quad-5.25839500 \quad-0.73373900-0.11788500$

C $\quad-4.32223600 \quad-1.35872200 \quad 0.72596400$

H $\quad-3.09293500 \quad-1.15706900 \quad 2.49293600$

H $\quad-3.69605800 \quad 1.23916300 \quad 2.85491700$

H $\quad-5.37837400 \quad 2.32246000 \quad 1.38452800$

H $\quad-6.37850800 \quad 1.05924300 \quad-0.51688400$

H $\quad-5.68185700 \quad-1.28869900 \quad-0.95208500$

H $\quad-4.09518200 \quad-2.41693500 \quad 0.59509000$

Ru -1.79957300 -1.11810900 -0.11856000

C $4.43461000 \quad-0.19168200 \quad 0.08353300$

C $5.88381100-0.57302800 \quad 0.21401300$

C $6.31057000-1.60641000 \quad 0.91032700$

C $4.24159800 \quad 1.30800200 \quad 0.29388900$

S $3.92612000 \quad-0.40209200 \quad-1.76669600$

C $6.74216100 \quad-2.62830000 \quad 1.61159200$

$\begin{array}{llll}\text { O } & 3.34901300 & 1.79987300 & 0.95337700\end{array}$

O $5.16060600 \quad 2.02237700 \quad-0.35648300$

C $5.02144700 \quad 3.44184500 \quad-0.25789000$

C $3.91254700 \quad-2.25655400 \quad-1.87348000$

H $6.59900100 \quad 0.03752000-0.33954600$

H $7.02501000 \quad-2.51326500 \quad 2.65796100$ 


$\begin{array}{rrrr}\text { H } & 6.83540500 & -3.62048700 & 1.16968000 \\ \mathrm{C} & 3.50493400 & -0.97314700 & 0.99062300 \\ \mathrm{H} & 4.82640200 & -2.65548500 & -1.41917000 \\ \mathrm{H} & 3.02368200 & -2.67763600 & -1.39570600 \\ \mathrm{H} & 3.90532200 & -2.49647700 & -2.93957000 \\ \mathrm{H} & 5.85721800 & 3.86550700 & -0.81727400 \\ \mathrm{H} & 4.06961500 & 3.76257800 & -0.69604400 \\ \mathrm{H} & 5.05895300 & 3.75933400 & 0.78911800 \\ \mathrm{H} & 3.63944500 & -2.05149300 & 0.85253700 \\ \mathrm{H} & 3.72265500 & -0.73938200 & 2.04096400 \\ \mathrm{H} & 2.45887600 & -0.71520500 & 0.78756600\end{array}$

\subsection{O-Complexe bimolecular}

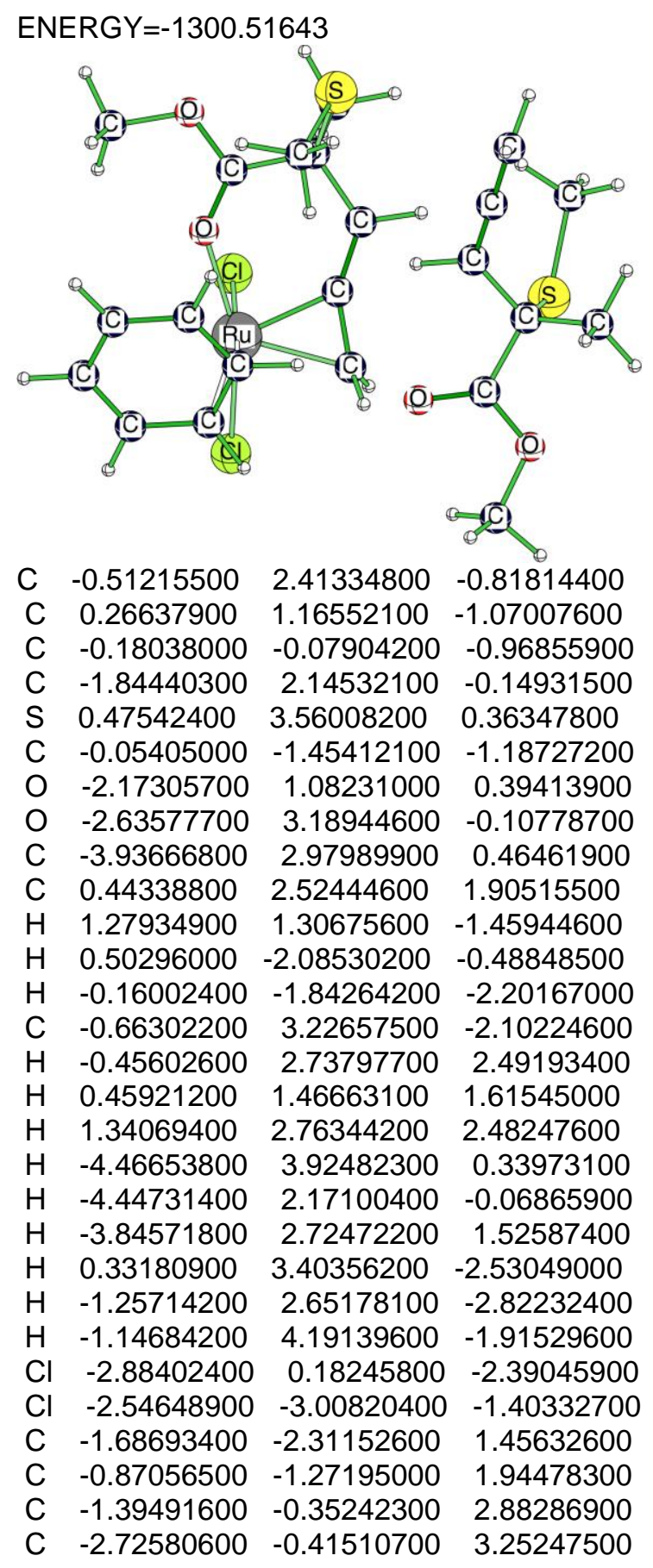

$\begin{array}{rrrr}\text { C } & -3.57117600 & -1.40680400 & 2.70379100 \\ \text { C } & -3.05481900 & -2.36138800 & 1.84414200 \\ \text { H } & -1.24854600 & -3.15789400 & 0.93168300 \\ \text { H } & 0.19523900 & -1.27056600 & 1.71179600 \\ \text { H } & -0.73830500 & 0.39580100 & 3.32372500 \\ \text { H } & -3.12552700 & 0.29671600 & 3.97330300 \\ \text { H } & -4.62130300 & -1.44277000 & 2.98804800 \\ \text { H } & -3.68303500 & -3.15996000 & 1.45554300 \\ \text { Ru } & -1.93167900 & -0.86982000 & -0.40791500 \\ \text { C } & 4.02848300 & -0.50794100 & 0.16185300 \\ \text { C } & 3.37311900 & 0.57590100 & 0.95787700 \\ \text { C } & 4.00259200 & 1.59729700 & 1.49649900 \\ \text { C } & 3.30270700 & -1.82328900 & 0.43266800 \\ \text { S } & 3.64856200 & -0.23132500 & -1.71530200 \\ \text { C } & 4.61149300 & 2.63363500 & 2.02443700 \\ \text { O } & 2.29883300 & -1.93632400 & 1.10753900 \\ \text { O } & 3.89670600 & -2.84203200 & -0.18402300 \\ \text { C } & 3.25006000 & -4.11144700 & -0.04678600 \\ \text { C } & 4.02268600 & 1.58305500 & -1.88113600 \\ \text { H } & 2.28488300 & 0.50906700 & 1.04449300 \\ \text { H } & 4.98753900 & 2.61131700 & 3.04716500 \\ \text { H } & 4.75621800 & 3.54981100 & 1.45043100 \\ \text { C } & 5.52977200 & -0.62006100 & 0.37536000 \\ \text { H } & 3.37288200 & 2.18109700 & -1.23213800 \\ \text { H } & 5.07415600 & 1.78805900 & -1.65746700 \\ \text { H } & 3.82067700 & 1.82279900 & -2.92907800 \\ \text { H } & 3.84851500 & -4.81588000 & -0.62655300 \\ \text { H } & 3.21493900 & -4.41080400 & 1.00575500 \\ \text { H } & 2.22957400 & -4.06421800 & -0.44270500 \\ \text { H } & 5.95421200 & -1.39591400 & -0.26757300 \\ \text { H } & 6.01762200 & 0.33476400 & 0.14662200 \\ \text { H } & 5.74631500 & -0.86255100 & 1.42479500\end{array}$

\subsection{TSso2 bimolecular}

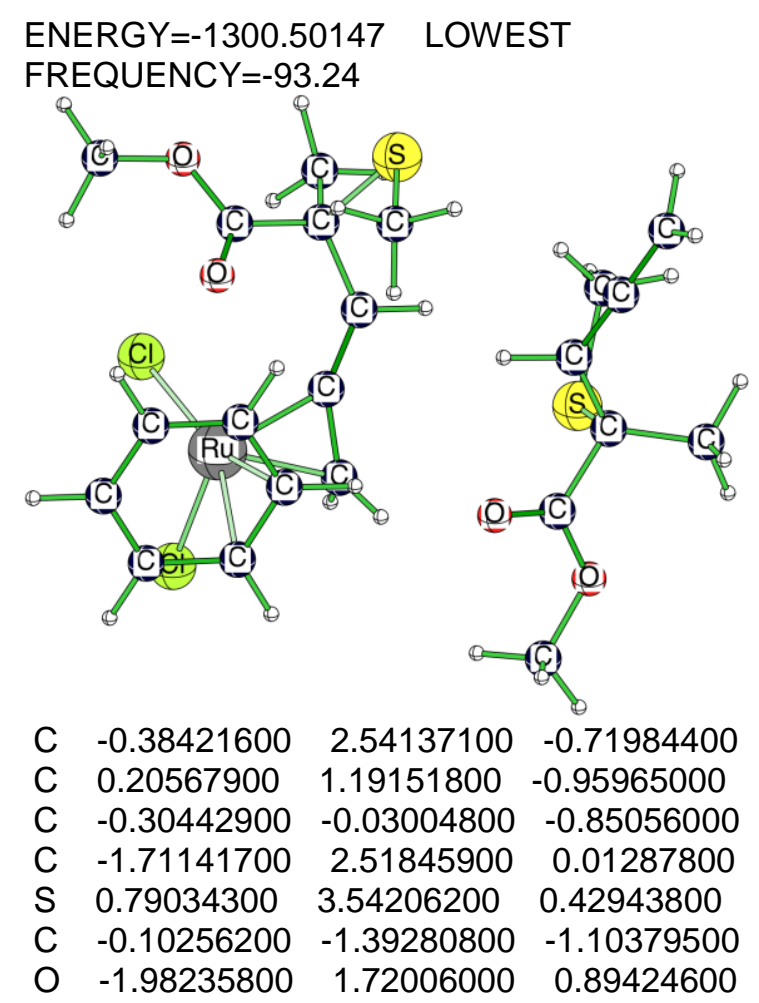




\begin{tabular}{|c|c|c|c|}
\hline & -2.51268500 & 3.51402200 & -0.34671800 \\
\hline & -3.79221500 & 3.52738400 & 0.28968700 \\
\hline & 0.75416300 & 2.51493500 & 1.97796400 \\
\hline & 1.21989500 & 1.21780300 & -1.37645400 \\
\hline & 0.53918800 & -1.99978200 & -0.45876500 \\
\hline & -0.24104800 & -1.76889100 & \\
\hline & -0.40394300 & 3.33158600 & -2.02375400 \\
\hline & -0.12492200 & 2.75701300 & 2.58074500 \\
\hline & 0.73773800 & 1.45295400 & 1.70580400 \\
\hline & 1.67132800 & 2.74361000 & 2.52840000 \\
\hline & -4.34439800 & 4.34988500 & -0.16871600 \\
\hline & -4.30334100 & 2.57400100 & 0.11240400 \\
\hline & -3.68336600 & 3.69356500 & 9300 \\
\hline & 0.59254100 & 3.30406800 & -2.48371500 \\
\hline & -1.12211100 & 2.87202700 & -2.71343900 \\
\hline & -0.68832900 & 4.37626300 & -1.85825000 \\
\hline & -3.15408100 & 0.47196800 & 3183100 \\
\hline & -2.66975800 & -2.76192600 & -1.68981300 \\
\hline & -1.67153300 & -2.65551000 & 1.27550000 \\
\hline & 2300 & 1900 & 0200 \\
\hline & 89400 & 166100 & 2.521 \\
\hline & 78200 & -0.5 & 2.68 \\
\hline & -3.73759200 & 7894800 & 946400 \\
\hline & -3.07 & 10700 & 51100 \\
\hline & -1.15150700 & -3.49569300 & 0.81897100 \\
\hline & 0.13940500 & -1.52353300 & 1.70576600 \\
\hline & 66500 & 57700 & 9600 \\
\hline & 7900 & 4900 & 7200 \\
\hline & -4.81927400 & -1.71243500 & 2.24804700 \\
\hline & -3.63941700 & -3.55560400 & 1.08693500 \\
\hline & $-1.97-2$ & -0.97 & $-0.2 c$ \\
\hline & 4.0464 & -0.63 & 0.08 \\
\hline & 3.513 & 0.45 & 0.944 \\
\hline & & & \\
\hline & 3.25 & -1.91 & 0.3 \\
\hline & 3.566 & -0.29 & 2700 \\
\hline & $4.94 C$ & 2.40 & 3900 \\
\hline & & -1.97 & \\
\hline & $3.744 \mathrm{~s}$ & -2.95388700 & 48000 \\
\hline & 3.04008700 & -4.18745800 & -0.12665600 \\
\hline & & 1.52 & 7600 \\
\hline & & & \\
\hline & 5.37810500 & 2.33 & 16300 \\
\hline & 5.10367100 & 3.32947900 & 1.41986900 \\
\hline & & -0.84433400 & \\
\hline & & & -1.22 \\
\hline & 5.007 & 1.71505100 & -1.65 \\
\hline & 3.75126700 & 1.78750500 & -2.92700300 \\
\hline & 3.58940600 & -4.92867400 & -0.70922700 \\
\hline & & -4.47140400 & 0.93035200 \\
\hline & 2.01451600 & -4.09749200 & -0.50309900 \\
\hline & 5.88599600 & -1.62825300 & -0.49331400 \\
\hline & 6.07511400 & 0.08537000 & -0.05813700 \\
\hline & & & \\
\hline
\end{tabular}

\section{5 cis-Allene bimolecular}

ENERGY $=-1300.51980$
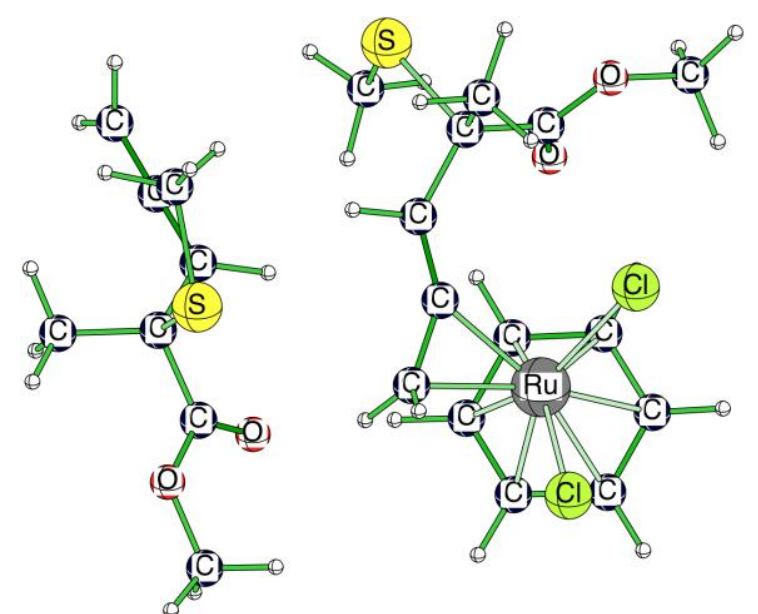

$\begin{array}{lll}\text { C } & 0.68013600 & 2.58 \\ \text { C } & -0.03035600\end{array}$

$2.58413900 \quad 0.76060900$

$\begin{array}{llll}\text { C } & -0.03035600 & 1.26586100 & 0.83305400\end{array}$

$\begin{array}{llll}\text { C } & 0.30336500 & -0.00947600 & 0.66701600\end{array}$

C $\quad 1.90760500 \quad 2.64321000 \quad-0.12806800$

S $\quad-0.51178100 \quad 3.89337000 \quad 0.01793000$

C $\quad-0.13326400 \quad-1.29806900 \quad 0.94254800$

$\begin{array}{llll}\text { O } & 1.95974300 & 2.17007000 & -1.24882400\end{array}$

$\begin{array}{llll}\text { O } & 2.88838600 & 3.36441500 & 0.41107100\end{array}$

C $\quad 4.09560800 \quad 3.41078200 \quad-0.34422600$

C $\quad-0.82887200 \quad 3.19794400 \quad-1.67558500$

H $\quad-1.02567600 \quad 1.36627100 \quad 1.28856200$

H $\quad-0.83452900 \quad-1.82556100 \quad 0.29451200$

H $\quad-0.02535400 \quad-1.68760700 \quad 1.95470400$

C $\quad 0.92791400 \quad 3.06463200 \quad 2.18890500$

H $\quad 0.05643600 \quad 3.30394300 \quad-2.30620500$

H $\quad-1.10503700 \quad 2.14153000 \quad-1.58550100$

H $\quad-1.67020400 \quad 3.76045900 \quad-2.08908900$

H $\quad 4.77832600 \quad 4.05025600 \quad 0.21884500$

H $\quad 4.51263300 \quad 2.39887000 \quad-0.43004800$

H $\quad 3.91757700 \quad 3.82512700 \quad-1.34272100$

H $\quad-0.00387700 \quad 2.99954200 \quad 2.76489300$

H $\quad 1.68079800 \quad 2.41668900 \quad 2.65331900$

H $\quad 1.28514700 \quad 4.10024500 \quad 2.20891000$

$\begin{array}{llll}\text { Cl } & 3.26873100 & 0.19044800 & 1.29558500\end{array}$

Cl $2.25201200-2.96838200 \quad 1.78377000$

C $1.41395900-2.95353800-1.48032700$

C $0.70121700-1.79405800-1.89133100$

C $1.39522100-0.57681800-2.12815100$

C $2.78402100-0.51487400-1.88927400$

C $3.52006900-1.70629700-1.57091600$

C $2.84158200-2.91829000-1.40534100$

H $\quad 0.87539900-3.86510800-1.23266800$

H $\quad-0.38692300-1.82000800-1.94528600$

H $\quad 0.85233700 \quad 0.33877600 \quad-2.34661400$

H $\quad 3.28222100 \quad 0.44984600-1.93258000$

H $4.58895300-1.64772200-1.38545500$

H $3.38168700 \quad-3.79988500-1.06996600$

Ru $1.82753000 \quad-1.32531100 \quad-0.02154100$

C $-3.97320700-0.46152400 \quad-0.08984100$

C $-3.36666400 \quad 0.55537400 \quad-1.00570300$

C $\quad-4.00376200 \quad 1.61008800 \quad-1.46578500$

C $\quad-3.36120500-1.82571200 \quad-0.39121500$

S $\quad-3.38433400 \quad-0.11104900 \quad 1.71801100$

C $\quad-4.63216200 \quad 2.67925000-1.89526700$

$\begin{array}{llll}\text { O } & -2.53805500 & -2.04009200 & -1.26068900\end{array}$ 


$\begin{array}{rrrr}\text { O } & -3.83008200 & -2.76469700 & 0.42497900 \\ \mathrm{C} & -3.26707400 & -4.07035400 & 0.26174400 \\ \mathrm{C} & -3.72223400 & 1.71405600 & 1.83906100 \\ \mathrm{H} & -2.30825200 & 0.41213300 & -1.24754900 \\ \mathrm{H} & -5.16370700 & 2.68671600 & -2.84653700 \\ \mathrm{H} & -4.63095200 & 3.59775000 & -1.30560200 \\ \mathrm{C} & -5.49393600 & -0.50938300 & -0.13543300 \\ \mathrm{H} & -3.07424100 & 2.28830300 & 1.16621700 \\ \mathrm{H} & -4.77557800 & 1.92829400 & 1.63239600 \\ \mathrm{H} & -3.49713300 & 1.97619700 & 2.87711000 \\ \mathrm{H} & -3.78064800 & -4.70907200 & 0.98173900 \\ \mathrm{H} & -3.42747700 & -4.43249900 & -0.75875800 \\ \mathrm{H} & -2.19171800 & -4.05099600 & 0.47545700 \\ \mathrm{H} & -5.88008400 & -1.24309800 & 0.57773300 \\ \mathrm{H} & -5.91043100 & 0.47510400 & 0.10932000 \\ \mathrm{H} & -5.83346800 & -0.77232700 & -1.14659600\end{array}$

\subsection{TSso3 bimolecular}

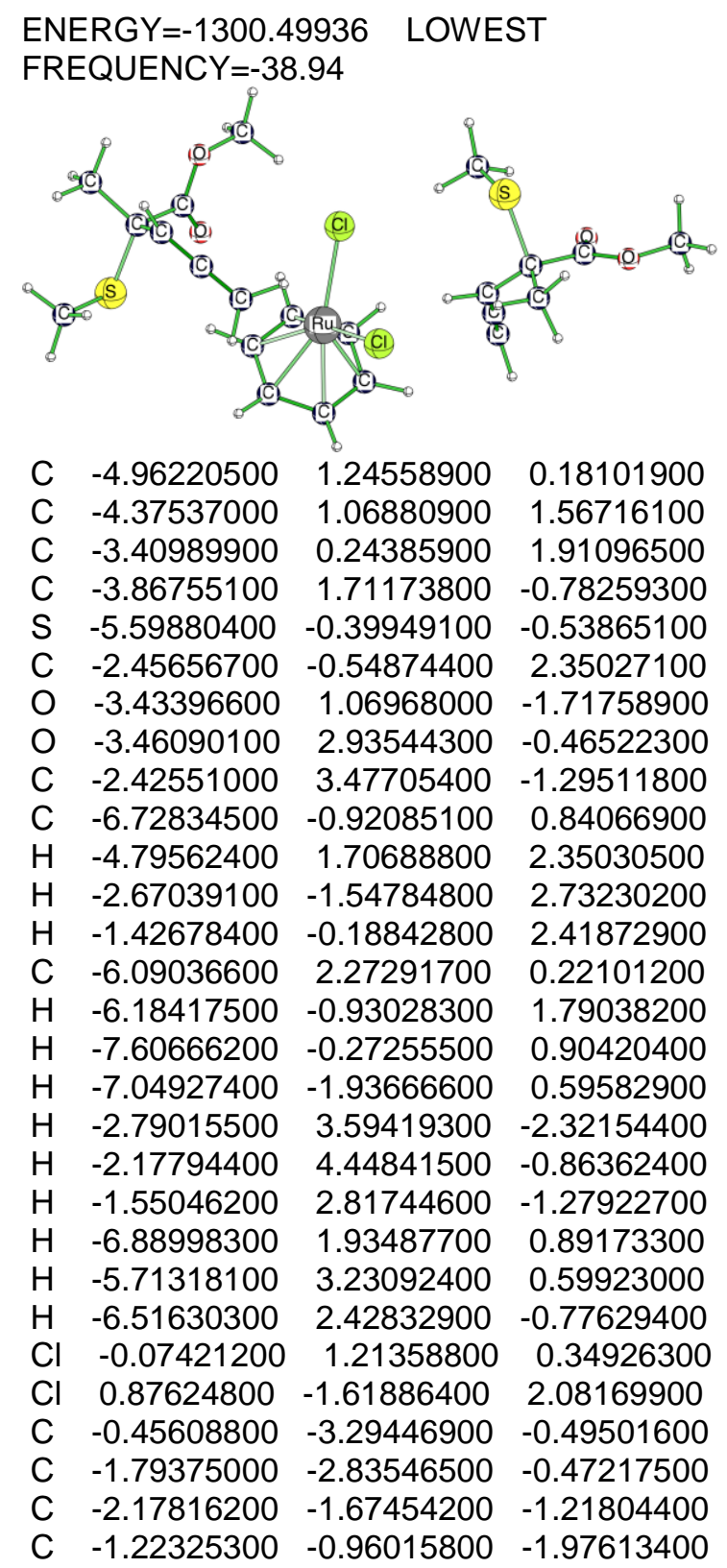

C $0.14082600 \quad-1.36753600 \quad-1.93449600$

C $0.52353400 \quad-2.53519500 \quad-1.19890400$

H $\quad-0.14336500 \quad-4.10285600 \quad 0.16001400$

H $\quad-2.51651400 \quad-3.30253700 \quad 0.19337900$

H $\quad-3.18458800 \quad-1.26611200-1.10973900$

H $\quad-1.51115300 \quad-0.00999100-2.41928000$

H $\quad 0.91918600 \quad-0.74144000 \quad-2.36875300$

H $\quad 1.58127000 \quad-2.77319700 \quad-1.09330600$

$\mathrm{Ru}-0.48342300 \quad-1.16965200 \quad 0.10077900$

C $4.53916400 \quad 0.26083800 \quad 0.57233900$

C $3.49761600 \quad-0.40820400 \quad-0.27534600$

C $3.64895300 \quad-0.88260700 \quad-1.49285300$

C $5.82644900 \quad 0.56236600 \quad-0.17562000$

S $3.91301300 \quad 1.98507500 \quad 1.11875800$

C $3.74845100 \quad-1.38526300 \quad-2.70349200$

O $5.88857200 \quad 0.92773100 \quad-1.33199500$

$\begin{array}{lllll}\text { O } & 6.90094200 & 0.43004900 & 0.61335000\end{array}$

C $8.14103700 \quad 0.80756100 \quad 0.01896600$

C $3.25561000 \quad 2.64822800 \quad-0.49071800$

H $2.52858300 \quad-0.53041500 \quad 0.22763500$

H $3.53761700 \quad-0.77854700 \quad-3.58472200$

H $4.08609400 \quad-2.40925100 \quad-2.86589600$

C $4.72386300 \quad-0.51992600 \quad 1.86864600$

H $4.01354100 \quad 2.53450900 \quad-1.27208800$

H $2.32650000 \quad 2.12931200 \quad-0.75163500$

H $3.05065200 \quad 3.70796900-0.31539200$

H $8.11505600 \quad 1.85761600 \quad-0.29210200$

H $8.90173400 \quad 0.65926900 \quad 0.78842900$

H $8.35772200 \quad 0.18402100 \quad-0.85520500$

H $3.74168400 \quad-0.69173900 \quad 2.32562000$

H $5.19069800-1.49043000 \quad 1.65891500$

H $5.35707900 \quad 0.02433900 \quad 2.57815200$

\section{7 cis-Allene-MLn-A-intermediary bimolecular}

ENERGY =-1300.50213
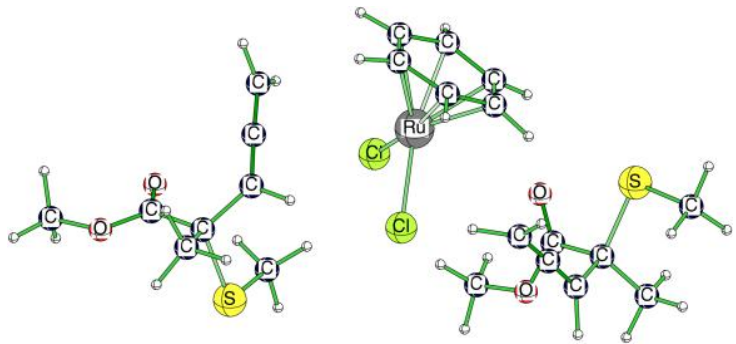

C $4.65423000-1.52774900-0.17103100$

C $4.05219300-2.22002700 \quad 1.03707900$

C $3.41503000-1.62825300 \quad 2.02380100$

C $3.52291800-1.21358400-1.15612900$

S $5.41468600 \quad 0.15798600 \quad 0.25112400$

C $2.76210000-1.07137900 \quad 3.01663900$

O $3.23223000-0.10312600-1.55019200$

O $2.90987100 \quad-2.33213100 \quad-1.52727700$

C $\quad 1.77101400 \quad-2.17894400 \quad-2.38994800$

C $6.69794500-0.38629300 \quad 1.48040900$

H $4.09901800 \quad-3.31281700 \quad 1.02697600$

H $3.26119300 \quad-0.78144900 \quad 3.94129300$

H $\quad 1.68562200 \quad-0.90860000 \quad 2.91777200$

C $5.68245400-2.43551900-0.84227400$ 


$\begin{array}{cccc}\mathrm{H} & 6.23927500 & -1.00435400 & 2.25856900 \\ \mathrm{H} & 7.51175400 & -0.92581700 & 0.98809200 \\ \mathrm{H} & 7.09301000 & 0.52901800 & 1.92877800 \\ \mathrm{H} & 2.02706300 & -1.56263200 & -3.25781500 \\ \mathrm{H} & 1.50048100 & -3.19014500 & -2.70000900 \\ \mathrm{H} & 0.94410100 & -1.71242300 & -1.83928600 \\ \mathrm{H} & 6.49545400 & -2.67746100 & -0.14692600 \\ \mathrm{H} & 5.20846000 & -3.37780500 & -1.14432000 \\ \mathrm{H} & 6.11082400 & -1.95558300 & -1.72948800 \\ \mathrm{Cl} & -0.57008100 & 0.81343100 & -2.32312900 \\ \mathrm{Cl} & 0.04812700 & -0.86972700 & 0.63510900 \\ \mathrm{C} & 1.10880000 & 2.18432300 & 1.75504600 \\ \mathrm{C} & 2.18531000 & 1.93673500 & 0.86981900 \\ \mathrm{C} & 2.17262200 & 2.49697500 & -0.44804500 \\ \mathrm{C} & 1.10839600 & 3.34836300 & -0.86105600 \\ \mathrm{C} & 0.00578200 & 3.54656300 & 0.00237900 \\ \mathrm{C} & -0.00777800 & 2.93675400 & 1.29921700 \\ \mathrm{H} & 1.05247700 & 1.65316900 & 2.70171700 \\ \mathrm{H} & 2.96565900 & 1.21859100 & 1.12906400 \\ \mathrm{H} & 2.93013600 & 2.16982200 & -1.15644800 \\ \mathrm{H} & 1.05483800 & 3.68516700 & -1.89246800 \\ \mathrm{H} & -0.88795500 & 4.04701900 & -0.36215600 \\ \mathrm{H} & -0.91077000 & 2.98812700 & 1.90351300 \\ \mathrm{Ru} & 0.35201000 & 1.39487800 & -0.17127400 \\ \mathrm{C} & -4.50836500 & -0.91391000 & 0.94114500 \\ \mathrm{C} & -3.38946200 & 0.08374000 & 0.96653900 \\ \mathrm{C} & -3.33785900 & 1.25561900 & 0.37242800 \\ \mathrm{C} & -5.68822200 & -0.48938700 & 0.08410800 \\ \mathrm{~S} & -3.88499300 & -2.52406700 & 0.10730100 \\ \mathrm{C} & -3.23642500 & 2.42465400 & -0.21934800 \\ \mathrm{O} & -5.60365500 & 0.11990800 & -0.96136300 \\ \mathrm{O} & -6.85279900 & -0.91589800 & 0.59454900 \\ \mathrm{C} & -7.99717300 & -0.65576000 & -0.21656400 \\ \mathrm{C} & -3.03215500 & -1.82980700 & -1.39591700 \\ \mathrm{H} & -2.50979600 & -0.24595600 & 1.53180400 \\ \mathrm{H} & -2.83808700 & 2.48514100 & -1.23397400 \\ \mathrm{H} & -3.58927900 & 3.34008900 & 0.25764800 \\ \mathrm{C} & -4.88011900 & -1.32367100 & 2.36123600 \\ \mathrm{H} & -3.71381500 & -1.16087300 & -1.93107200 \\ \mathrm{H} & -2.12226400 & -1.29925700 & -1.09701600 \\ \mathrm{H} & -2.77994200 & -2.69176100 & -2.02010000 \\ \mathrm{H} & -7.87888000 & -1.11108000 & -1.20587900 \\ \mathrm{H} & -8.84586000 & -1.10050800 & 0.30748000 \\ \mathrm{H} & -8.14709000 & 0.42256900 & -0.33877100 \\ \mathrm{H} & -3.97159300 & -1.60901200 & 2.90493600 \\ \mathrm{H} & -5.35539400 & -0.48487400 & 2.88485800 \\ \mathrm{H} & -5.57156300 & -2.17327000 & 2.36786200\end{array}$

\subsection{TSso4 bimolecular}

ENERGY $=-1300.50156 \quad$ LOWEST

FREQUENCY $=-26.46$
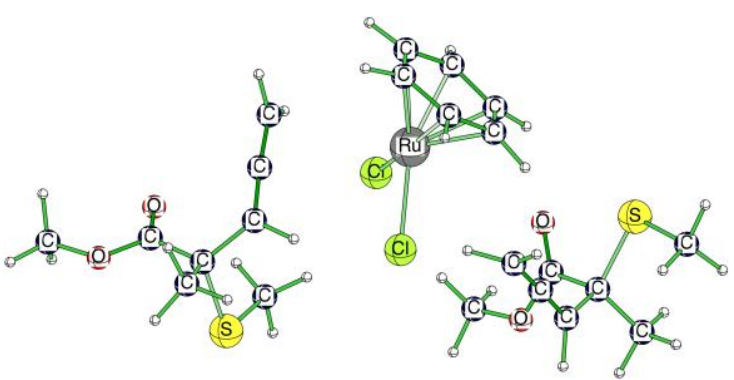

C $4.51253700-1.65643900-0.14946000$

C $3.80674200-2.30798000 \quad 1.02407200$

C $3.22146300-1.67740600 \quad 2.01823900$

C $3.45950200-1.27412000-1.19536900$

S $5.35457700 \quad-0.02035500 \quad 0.31154300$

C $2.61973600-1.07441800 \quad 3.01638600$

$\begin{array}{llll}\text { O } & 3.27578000 & -0.15223200 & -1.61934400\end{array}$

$\begin{array}{llll}\text { O } & 2.78074400 & -2.34641500 & -1.58701100\end{array}$

C $\quad 1.71362900-2.10171700-2.51785700$

C $\quad 6.52432800-0.63867900 \quad 1.61724900$

H $\quad 3.72832300 \quad-3.39814100 \quad 0.97910300$

H $3.12935600 \quad-0.87068800 \quad 3.95818000$

H $\quad 1.57217300 \quad-0.78430600 \quad 2.90108400$

$\begin{array}{llll}\text { C } \quad 5.51587600 & -2.62751500 & -0.76826800\end{array}$

H $\quad 5.98169100 \quad-1.22898900 \quad 2.36259100$

H $\quad 7.33340600 \quad-1.22646700 \quad 1.17484100$

H $\quad 6.94415000 \quad 0.25151300 \quad 2.09278000$

H $2.11393400-1.69059700-3.45096200$

H $\quad 1.24932400-3.07405500-2.69312200$

H $\quad 0.98866300 \quad-1.39825800-2.09059200$

H $\quad 6.28226700-2.90672200-0.03507300$

H $5.00405800-3.54506500-1.08469200$

H $\quad 6.00915900 \quad-2.17985700-1.63859100$

Cl $\quad-0.49440500 \quad 0.97198300 \quad-2.37548000$

Cl $0.05614500 \quad-0.76618000 \quad 0.55210300$

C $1.14892000 \quad 2.27333400 \quad 1.76055000$

C $\quad 2.16852900 \quad 1.89095000 \quad 0.85074700$

$\begin{array}{llll}\text { C } & 2.19409600 & 2.43786700 & -0.47104800\end{array}$

C $\quad 1.22160500 \quad 3.40457600 \quad-0.86767600$

$\begin{array}{llll}\text { C } & 0.17984800 & 3.75075800 & 0.02192900\end{array}$

C $\quad 0.12825500 \quad 3.15849400 \quad 1.32564700$

H $\quad 1.05627100 \quad 1.76706500 \quad 2.71807700$

H $\quad 2.87002700 \quad 1.09281900 \quad 1.10481500$

H $2.89793000 \quad 2.02461500-1.19074400$

H $\quad 1.18285800 \quad 3.73550700 \quad-1.90177700$

H $\quad-0.64689300 \quad 4.36609800 \quad-0.32415900$

H $\quad-0.73977300 \quad 3.33023500 \quad 1.95763000$

Ru $0.27168600 \quad 1.55753200 \quad-0.14297600$

C $\quad-4.41699300 \quad-1.03706100 \quad 0.93103500$

C $\quad-3.24746900-0.10493100 \quad 0.98786600$

C $\quad-3.12710200 \quad 1.09519100 \quad 0.46974000$

C $\quad-5.59681400-0.49875000 \quad 0.14071300$

S $\quad-3.88221200-2.59995900-0.04562000$

C $\quad-2.91278100 \quad 2.28491600-0.05409500$

$\begin{array}{llll}\text { O } & -5.51079700 & 0.20612400 & -0.84299900\end{array}$

$\begin{array}{llll}O & -6.76293600 & -0.94461100 & 0.62844400\end{array}$

C $-7.91224100-0.59000100-0.13904200$

C $-3.01142600-1.82400600-1.50005000$

H $\quad-2.35846600-0.52551800 \quad 1.47440000$

$\mathrm{H} \quad-2.57081900 \quad 2.36898600 \quad-1.08741900$

H $\quad-3.14960600 \quad 3.19993200 \quad 0.49172200$ 


$\begin{array}{rrrr}\mathrm{C} & -4.76971200 & -1.53775900 & 2.32612500 \\ \mathrm{H} & -3.65807800 & -1.06657400 & -1.95433500 \\ \mathrm{H} & -2.06217800 & -1.38211400 & -1.17866300 \\ \mathrm{H} & -2.83337900 & -2.63820700 & -2.20823100 \\ \mathrm{H} & -7.82539600 & -0.97990500 & -1.15911400 \\ \mathrm{H} & -8.76602300 & -1.04281500 & 0.36947200 \\ \mathrm{H} & -8.02671900 & 0.49843000 & -0.18386200 \\ \mathrm{H} & -3.85992200 & -1.89602900 & 2.82217600 \\ \mathrm{H} & -5.19907500 & -0.72237200 & 2.92140200 \\ \mathrm{H} & -5.49435900 & -2.35829000 & 2.28932300\end{array}$

\subsection{Ru-Ln-A bimolecular}

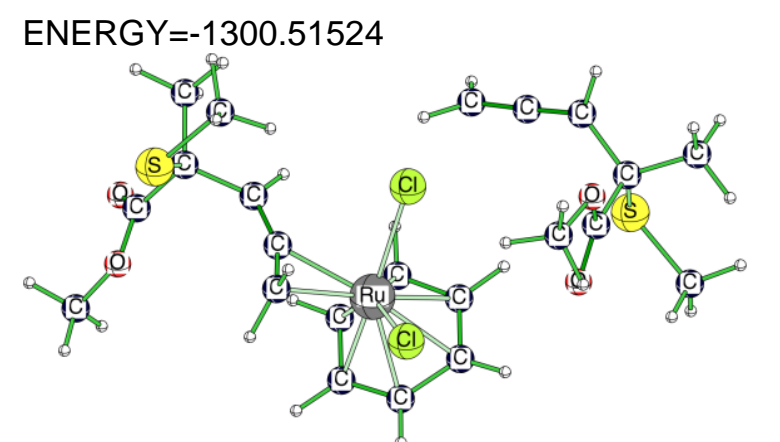

C $\quad 4.71954300 \quad 1.26117300 \quad-0.24960800$

C $3.81874800 \quad 2.47859600 \quad-0.33105100$

C $2.72175400 \quad 2.57141800 \quad-1.05055700$

$\begin{array}{llll}\text { C } & 4.10269700 & 0.27125500 & 0.75128500\end{array}$

S $4.78270900 \quad 0.51538700 \quad-1.98333400$

C $1.61484100 \quad 2.70636800-1.74378400$

$\begin{array}{llll}\text { O } & 3.75080800 & -0.86378900 & 0.50489700\end{array}$

$\begin{array}{llll}\text { O } & 4.02105700 & 0.83388900 & 1.95286600\end{array}$

C $3.35411800 \quad 0.05845100 \quad 2.96257300$

$\begin{array}{llll}\text { C } & 5.96924500 & -0.89737900 & -1.74385600\end{array}$

H $\quad 4.09681200 \quad 3.31628700 \quad 0.31399600$

H $\quad 1.61605500 \quad 3.11684400 \quad-2.75327800$

$\begin{array}{llll}\mathrm{H} & 0.66737800 & 2.40987300 & -1.28522800\end{array}$

C $\quad 6.11144600 \quad 1.67432900 \quad 0.22315900$

H $5.60663200-1.55705800-0.95181900$

H $5.98712200-1.43780400-2.69409300$

H $6.97516100-0.52923400-1.52242300$

$\begin{array}{llll}H & 3.84160900 & -0.91546700 & 3.07846900\end{array}$

H $3.43765300 \quad 0.64103700 \quad 3.88225900$

H $2.30027800 \quad-0.08619600 \quad 2.69014200$

H $\quad 6.56652700 \quad 2.34709600 \quad-0.51297100$

$\begin{array}{llll}\mathrm{H} & 6.04061800 & 2.19819200 & 1.18428100\end{array}$

H $\quad 6.76871100 \quad 0.80778800 \quad 0.36401300$

Cl $0.31845800-2.04064800 \quad 2.44177700$

Cl $\quad 0.35264800 \quad 1.03305100 \quad 1.09256800$

C $\quad 0.13988400 \quad-0.73907100-1.92732100$

C $1.37195800-1.18978600-1.35985400$

C $1.39666500-2.39050000-0.64389800$

C $0.20520000-3.18391000-0.51640200$

C $\quad-0.97607300-2.81861000-1.19623000$

C $\quad-1.00732000-1.57747500 \quad-1.89183000$

H $\quad 0.09078900 \quad 0.23830900-2.40360900$

H $\quad 2.26265600 \quad-0.55874500-1.38445900$

H $\quad 2.28643000 \quad-2.65241700 \quad-0.07868400$

H $\quad 0.21144600 \quad-4.05792800 \quad 0.12881500$

H $\quad-1.87660100 \quad-3.42001500-1.10269900$

$\begin{array}{lrrr}\text { H } & -1.94307700 & -1.22597800 & -2.32068200 \\ \mathrm{Ru} & -0.35768300 & -1.15711100 & 0.20722900 \\ \mathrm{C} & -4.10197000 & 1.46424300 & -0.33343000 \\ \mathrm{C} & -2.72440300 & 0.84665300 & -0.51978800 \\ \mathrm{C} & -2.24436500 & -0.13651300 & 0.22370500 \\ \mathrm{C} & -5.05626800 & 0.60436800 & -1.16292800 \\ \mathrm{~S} & -4.69370500 & 1.42243700 & 1.46918500 \\ \mathrm{C} & -2.27040000 & -1.00053600 & 1.29386600 \\ \mathrm{O} & -5.37712000 & 0.86593200 & -2.30278700 \\ \mathrm{O} & -5.43266400 & -0.50437700 & -0.52155800 \\ \mathrm{C} & -6.26878300 & -1.38772900 & -1.26983900 \\ \mathrm{C} & -3.39016600 & 2.50602000 & 2.23716200 \\ \mathrm{H} & -2.13019100 & 1.27878900 & -1.33143100 \\ \mathrm{H} & -2.03553600 & -0.62173200 & 2.28997700 \\ \mathrm{H} & -2.79908000 & -1.95356400 & 1.24474100 \\ \mathrm{C} & -4.09325800 & 2.88812900 & -0.87172500 \\ \mathrm{H} & -2.39409600 & 2.18438900 & 1.90892900 \\ \mathrm{H} & -3.55697800 & 3.55974300 & 1.99808100 \\ \mathrm{H} & -3.47783000 & 2.35814000 & 3.31681800 \\ \mathrm{H} & -5.75027100 & -1.74082200 & -2.16864000 \\ \mathrm{H} & -6.49311900 & -2.22428500 & -0.60547200 \\ \mathrm{H} & -7.19181900 & -0.88059000 & -1.56874900 \\ \mathrm{H} & -3.34119700 & 3.48878000 & -0.34697500 \\ \mathrm{H} & -3.84101100 & 2.88434000 & -1.93941800 \\ \mathrm{H} & -5.07511700 & 3.35886500 & -0.75063800\end{array}$

\subsection{TSso5 bimolecular}

ENERGY=-1300.50134 LOWEST
FREQUENCY=-223.89

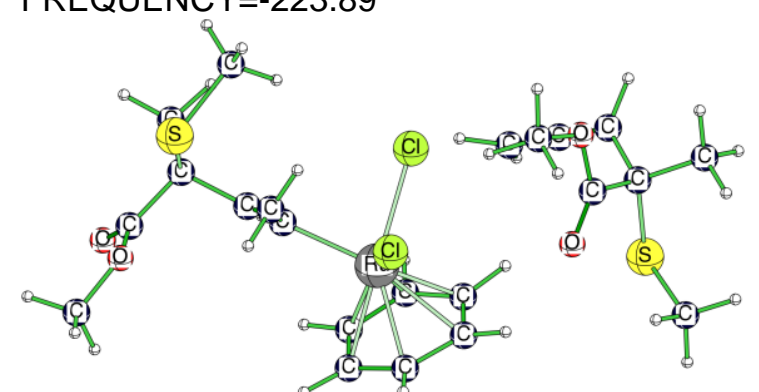

C 4.98630800

$0.86183900-0.41016800$

C $\quad 4.38208200 \quad 1.92534700 \quad-1.30651200$

C $3.37846500 \quad 1.73421200-2.13496800$

$\begin{array}{llll}\text { C } & 4.06694900 & 0.62866400 & 0.79929700\end{array}$

S $5.14158700 \quad-0.70100400-1.46256100$

C $\quad 2.36485300 \quad 1.57487500 \quad-2.95321400$

$\begin{array}{llll}\text { O } & 3.65252200 & -0.44470500 & 1.18283300\end{array}$

$\begin{array}{llll}\text { O } & 3.82497200 & 1.78301100 & 1.41260500\end{array}$

$\begin{array}{llll}\text { C } 2.95974800 & 1.70847000 & 2.55672000\end{array}$

C $5.96117000-1.89106800-0.28931500$

H $\quad 4.79423000 \quad 2.93195700-1.19880000$

H $\quad 2.51130400 \quad 1.31613200-4.00165200$

H $\quad 1.35064000 \quad 1.70876600 \quad-2.56983100$

C $\quad 6.35326800 \quad 1.32012100 \quad 0.09559600$

H $\quad 5.35773100 \quad-1.98738500 \quad 0.61635800$

H $5.99933800-2.84794300-0.81715200$

H $\quad 6.98058900-1.57141400-0.05387500$

H $\quad 3.35669800 \quad 0.99623900 \quad 3.28823100$

H $2.93742100 \quad 2.71783700 \quad 2.97311700$

H $\quad 1.95616800 \quad 1.39424200 \quad 2.24521200$ 


$\begin{array}{cccc}\mathrm{H} & 7.03620100 & 1.45390300 & -0.75150300 \\ \mathrm{H} & 6.25246000 & 2.27610100 & 0.62499600 \\ \mathrm{H} & 6.79360000 & 0.59540400 & 0.79133000 \\ \mathrm{Cl} & -0.05884300 & -0.60952500 & 2.81337300 \\ \mathrm{Cl} & 0.35933600 & 1.50339200 & 0.04359600 \\ \mathrm{C} & 0.24449800 & -1.44177900 & -1.74375400 \\ \mathrm{C} & 1.42377500 & -1.51790300 & -0.93162200 \\ \mathrm{C} & 1.43629100 & -2.30169800 & 0.23451200 \\ \mathrm{C} & 0.24092900 & -2.97852200 & 0.63741000 \\ \mathrm{C} & -0.91532600 & -2.96893700 & -0.18228500 \\ \mathrm{C} & -0.90680400 & -2.18523800 & -1.37711300 \\ \mathrm{H} & 0.21932400 & -0.77406500 & -2.60228300 \\ \mathrm{H} & 2.28328500 & -0.88737100 & -1.16004800 \\ \mathrm{H} & 2.28939600 & -2.23361600 & 0.90395800 \\ \mathrm{H} & 0.19980100 & -3.45299300 & 1.61440700 \\ \mathrm{H} & -1.82674000 & -3.46101000 & 0.14588200 \\ \mathrm{H} & -1.82223300 & -2.08391500 & -1.95583200 \\ \mathrm{Ru} & -0.33682500 & -0.88222200 & 0.29211400 \\ \mathrm{C} & -4.33386000 & 1.02569200 & -0.82201300 \\ \mathrm{C} & -2.98559900 & 0.33128800 & -0.98752000 \\ \mathrm{C} & -2.25925000 & -0.00129400 & 0.07677700 \\ \mathrm{C} & -5.38808400 & -0.07453800 & -0.94215400 \\ \mathrm{~S} & -4.46153500 & 1.75972400 & 0.92389400 \\ \mathrm{C} & -2.50387300 & 0.26324500 & 1.42542900 \\ \mathrm{O} & -5.93039300 & -0.36663900 & -1.98507100 \\ \mathrm{O} & -5.57078700 & -0.72801800 & 0.20816500 \\ \mathrm{C} & -6.46835300 & -1.83971300 & 0.14841100 \\ \mathrm{C} & -3.31580000 & 3.21549400 & 0.74757500 \\ \mathrm{H} & -2.68489900 & 0.13472200 & -2.01984800 \\ \mathrm{H} & -1.95900700 & 1.06318200 & 1.92669700 \\ \mathrm{H} & -2.96650400 & -0.48199900 & 2.07303100 \\ \mathrm{C} & -4.54090000 & 2.08050400 & -1.89669500 \\ \mathrm{H} & -2.38948700 & 2.89822700 & 0.25306000 \\ \mathrm{H} & -3.80836700 & 4.01789500 & 0.19419100 \\ \mathrm{H} & -3.09543300 & 3.54678100 & 1.76563800 \\ \mathrm{H} & -6.09885900 & -2.58794700 & -0.56102900 \\ \mathrm{H} & -6.50219600 & -2.25297500 & 1.15799900 \\ \mathrm{H} & -7.46512600 & -1.51166300 & -0.16357700 \\ \mathrm{H} & -3.72510200 & 2.81140100 & -1.88014800 \\ \mathrm{H} & -4.53693500 & 1.59209100 & -2.87798900 \\ \mathrm{H} & -5.49966000 & 2.59839600 & -1.77798400\end{array}$

\subsection{S-Cycle bimolecular}

ENERGY $=-1300.51430$

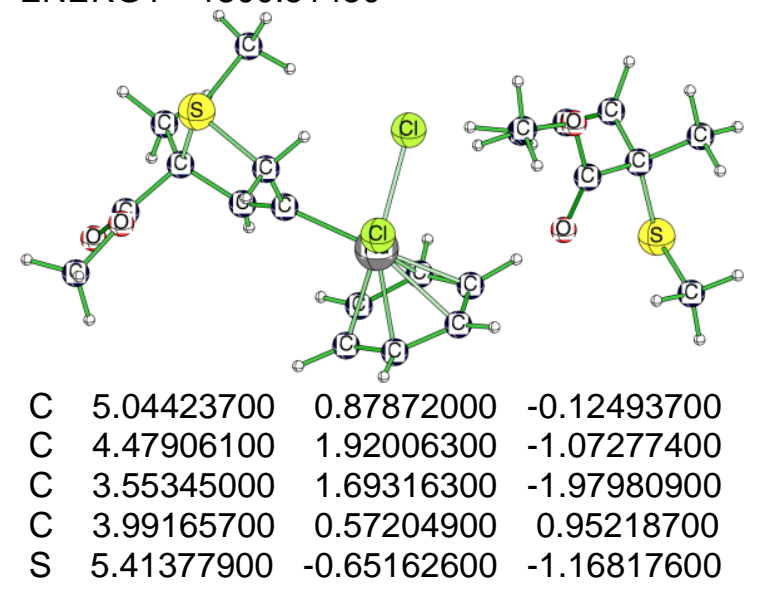

C $\quad 2.61101900 \quad 1.50177300 \quad-2.87259400$

$\begin{array}{llll}\text { O } & 3.49732900 & -0.51074200 & 1.18109100\end{array}$

$\begin{array}{llll}\text { O } & 3.70631200 & 1.68063400 & 1.63243700\end{array}$

C $2.66480500 \quad 1.54528600 \quad 2.61238800$

$\begin{array}{llll}\text { C } & 6.12765500 & -1.81554200 & 0.09695000\end{array}$

H $\quad 4.84683100 \quad 2.94028100 \quad-0.93575400$

H $\quad 2.84440300 \quad 1.24539400 \quad-3.90583500$

H $\quad 1.56723500 \quad 1.59810000 \quad-2.55838300$

C $\quad 6.31426500 \quad 1.41016900 \quad 0.53565400$

H $\quad 5.40949900 \quad-1.96496200 \quad 0.90729300$

H $\quad 6.29596000 \quad-2.76005000-0.42763700$

H $\quad 7.08336500 \quad-1.44376500 \quad 0.47858300$

H $\quad 2.94969500 \quad 0.80816100 \quad 3.37100700$

H $\quad 2.55255700 \quad 2.53515300 \quad 3.06044300$

H $\quad 1.73380500 \quad 1.22703700 \quad 2.12818600$

H $\quad 7.07607900 \quad 1.60219800 \quad-0.22924900$

H $\quad 6.09913300 \quad 2.34686700 \quad 1.06490400$

H $\quad 6.72112200 \quad 0.69925200 \quad 1.26521800$

$\begin{array}{llll}\mathrm{Cl} & -0.32936600 & -0.62371000 & 2.57644500\end{array}$

Cl $0.30349100 \quad 1.53612300-0.20115100$

C $0.52823600 \quad-1.41543400-1.87763700$

C $1.57619700-1.65316200-0.92403400$

C $1.32640000 \quad-2.45082800 \quad 0.19494800$

C $\quad 0.01872400 \quad-3.01672900 \quad 0.38454100$

C $\quad-0.98663900-2.88212500-0.60770900$

C $\quad-0.72420300-2.06860800 \quad-1.75499900$

H $\quad 0.69224700-0.70868300-2.68844200$

H $2.52133300 \quad-1.11571900-1.00249900$

H $\quad 2.06736700 \quad-2.50787900 \quad 0.98714100$

H $\quad-0.21426400-3.52233400 \quad 1.31836100$

H $\quad-1.97609100-3.30356200-0.44670700$

H $\quad-1.50546900-1.88716700 \quad-2.48907300$

$\begin{array}{lll}\text { Ru }-0.35889300 & -0.89763700 & 0.05393900\end{array}$

C $\quad-4.39770600 \quad 0.83120200 \quad-0.85766800$

C $\quad-3.12073500 \quad 0.02699900-0.98454500$

C $\quad-2.25125800 \quad-0.04209100 \quad 0.04169100$

C $\quad-5.52709000-0.14081700 \quad-0.52158600$

S $\quad-4.06588600 \quad 1.91724800 \quad 0.68610300$

C $\quad-2.63983600 \quad 0.73854600 \quad 1.25195000$

$\begin{array}{llll}\text { O } & -6.19036900 & -0.68501700 & -1.37473900\end{array}$

$\begin{array}{llll}O & -5.62011100 & -0.38174600 & 0.78672500\end{array}$

C $\quad-6.55679500 \quad-1.39814500 \quad 1.16558400$

C $\quad-2.99370800 \quad 3.25949500 \quad-0.02407400$

H $\quad-2.98750900-0.45689100-1.95304900$

H $\quad-1.85317900 \quad 1.40096400 \quad 1.62533400$

H $\quad-3.05855800 \quad 0.14156600 \quad 2.06649600$

C $\quad-4.73039400 \quad 1.66021300-2.08188400$

H $\quad-2.14855500 \quad 2.79421300-0.54635700$

H $\quad-3.60921900 \quad 3.88348500 \quad-0.67438200$

H $\quad-2.64046700 \quad 3.83574500 \quad 0.83417100$

H $\quad-6.27886300 \quad-2.35314400 \quad 0.70857600$

H $\quad-6.50294700-1.46430400 \quad 2.25312200$

H $\quad-7.56645400 \quad-1.12215900 \quad 0.84566800$

H $\quad-3.86144400 \quad 2.24312600 \quad-2.40528300$

H $\quad-4.99537600 \quad 0.96842700 \quad-2.88923700$

H $\quad-5.58667300 \quad 2.32549700 \quad-1.91671900$

4.12 TSso6 bimolecular 


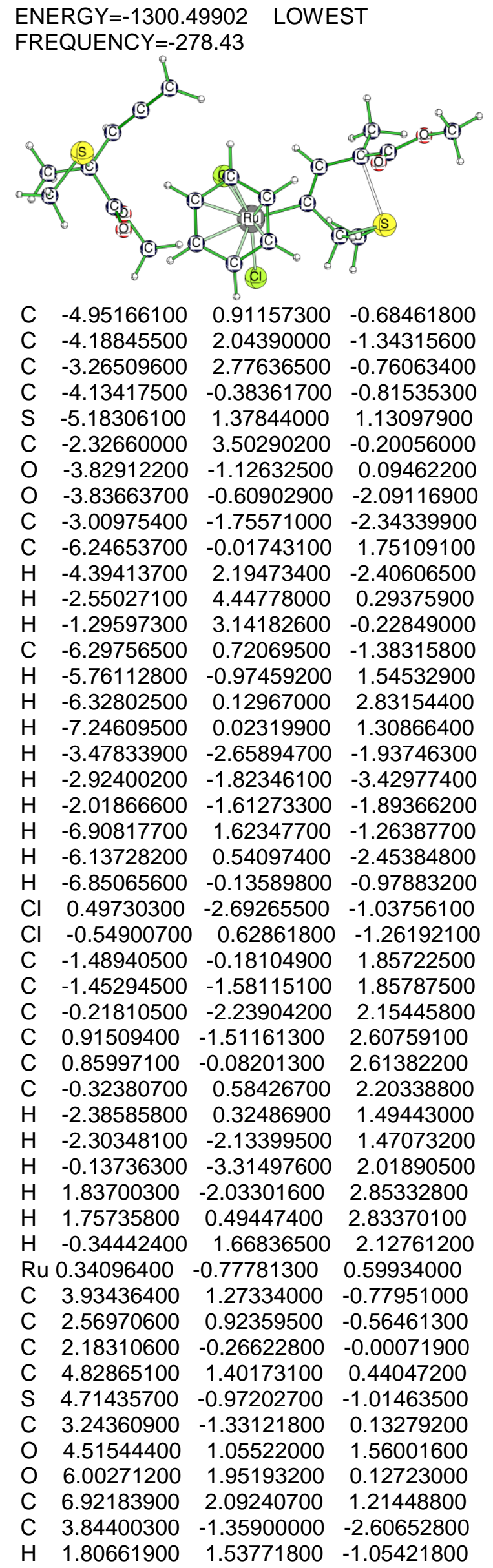

$\begin{array}{rrrr}H & 2.84670600 & -2.30761000 & -0.16962500 \\ H & 3.66056800 & -1.38323100 & 1.14629300 \\ \mathrm{C} & 4.28638100 & 2.10681100 & -1.97171000 \\ \mathrm{H} & 3.67427300 & -2.43749800 & -2.64887700 \\ \mathrm{H} & 2.87749800 & -0.83661200 & -2.60418400 \\ \mathrm{H} & 4.48433400 & -1.02895000 & -3.42683500 \\ \mathrm{H} & 7.18720100 & 1.10851800 & 1.61599200 \\ \mathrm{H} & 7.80334800 & 2.58503600 & 0.80059500 \\ \mathrm{H} & 6.48074800 & 2.69841700 & 2.01229200 \\ \mathrm{H} & 3.63917300 & 1.85921900 & -2.81974800 \\ \mathrm{H} & 4.13303000 & 3.16710300 & -1.72436200 \\ \mathrm{H} & 5.33695000 & 1.99447200 & -2.25564900\end{array}$

\subsection{TSso6 bimolecular}

ENERGY $=-1300.49902$ LOWEST FREQUENCY=-278.43

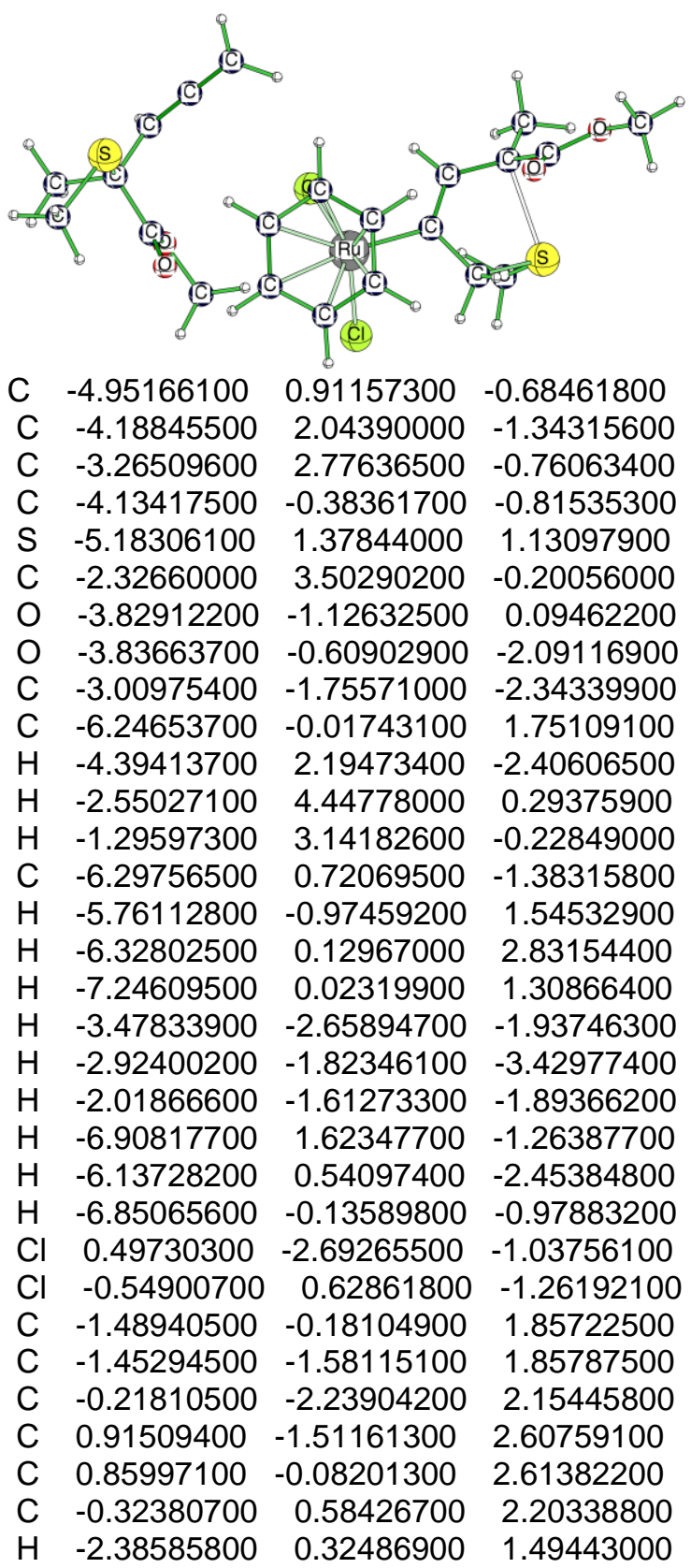



H $\quad-2.30348100 \quad-2.13399500 \quad 1.47073200$
H $\quad-0.13736300 \quad-3.31497600 \quad 2.01890500$
H $\quad 1.83700300 \quad-2.03301600 \quad 2.85332800$
$\begin{array}{llll}\mathrm{H} & 1.75735800 & 0.49447400 & 2.83370100\end{array}$
$\begin{array}{llll}\mathrm{H} & -0.34442400 & 1.66836500 & 2.12761200\end{array}$
Ru $0.34096400 \quad-0.77781300 \quad 0.59934000$
C $3.93436400 \quad 1.27334000 \quad-0.77951000$
$\begin{array}{llll}\text { C } & 2.56970600 & 0.92359500 & -0.56461300\end{array}$
C $2.18310600 \quad-0.26622800 \quad-0.00071900$
C $\quad 4.82865100 \quad 1.40173100 \quad 0.44047200$
S $4.71435700 \quad-0.97202700 \quad-1.01463500$
C $3.24360900 \quad-1.33121800 \quad 0.13279200$
$\begin{array}{llll}\mathrm{O} & 4.51544400 & 1.05522000 & 1.56001600\end{array}$
$\begin{array}{llll}\text { O } & 6.00271200 & 1.95193200 & 0.12723000\end{array}$
$\begin{array}{llll}\text { C } & 6.92183900 & 2.09240700 & 1.21448800\end{array}$

$\begin{array}{rrrr}\mathrm{C} & 3.84400300 & -1.35900000 & -2.60652800 \\ \mathrm{H} & 1.80661900 & 1.53771800 & -1.05421800 \\ \mathrm{H} & 2.84670600 & -2.30761000 & -0.16962500 \\ \mathrm{H} & 3.66056800 & -1.38323100 & 1.14629300 \\ \mathrm{C} & 4.28638100 & 2.10681100 & -1.97171000 \\ \mathrm{H} & 3.67427300 & -2.43749800 & -2.64887700 \\ \mathrm{H} & 2.87749800 & -0.83661200 & -2.60418400 \\ \mathrm{H} & 4.48433400 & -1.02895000 & -3.42683500 \\ \mathrm{H} & 7.18720100 & 1.10851800 & 1.61599200 \\ \mathrm{H} & 7.80334800 & 2.58503600 & 0.80059500 \\ \mathrm{H} & 6.48074800 & 2.69841700 & 2.01229200 \\ \mathrm{H} & 3.63917300 & 1.85921900 & -2.81974800 \\ \mathrm{H} & 4.13303000 & 3.16710300 & -1.72436200 \\ \mathrm{H} & 5.33695000 & 1.99447200 & -2.25564900\end{array}$

C $3.84400300 \quad-1.35900000-2.60652800$

H $2.84670600-2.30761000-0.16962500$

H $3.66056800 \quad-1.38323100 \quad 1.14629300$

$\begin{array}{llll}\mathrm{H} & 3.67427300 & -2.43749800 & -2.64887700\end{array}$

H $2.87749800 \quad-0.83661200-2.60418400$

H $\quad 4.48433400 \quad-1.02895000-3.42683500$

$\begin{array}{lll}H & 7.18720100 & 1.10851800-1.61599200\end{array}$

H $\quad 6.48074800 \quad 2.69841700 \quad 2.01229200$

H $\quad 5.33695000 \quad 1.99447200 \quad-2.25564900$ 